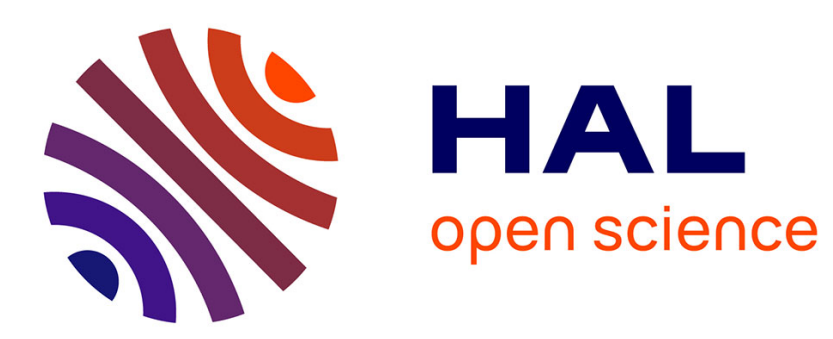

\title{
Biothérapies versus immunosuppresseurs conventionnels au cours des uvéites non-infectieuses non-antérieures : étude comparative rétrospective
}

Mathilde Leclercq

\section{- To cite this version:}

Mathilde Leclercq. Biothérapies versus immunosuppresseurs conventionnels au cours des uvéites noninfectieuses non-antérieures : étude comparative rétrospective. Médecine humaine et pathologie. 2019. dumas-02316184

\section{HAL Id: dumas-02316184 https://dumas.ccsd.cnrs.fr/dumas-02316184}

Submitted on 3 Dec 2019

HAL is a multi-disciplinary open access archive for the deposit and dissemination of scientific research documents, whether they are published or not. The documents may come from teaching and research institutions in France or abroad, or from public or private research centers.
L'archive ouverte pluridisciplinaire HAL, est destinée au dépôt et à la diffusion de documents scientifiques de niveau recherche, publiés ou non, émanant des établissements d'enseignement et de recherche français ou étrangers, des laboratoires publics ou privés. 
FACULTÉ MIXTE DE MÉDECINE ET DE PHARMACIE DE ROUEN

ANNÉE 2019

$\mathbf{N}^{\circ}$

\section{THÈSE POUR LE DOCTORAT EN MÉDECINE}

(Diplôme d'État)

PAR

\section{LECLERCQ Mathilde}

Née le 3 Mars 1990 à Amiens

PRÉSENTÉE ET SOUTENUE PUBLIQUEMENT LE 20 SEPTEMBRE 2019

\section{Biothérapies versus immunosuppresseurs conventionnels au cours des uvéites non-infectieuses non-antérieures : étude comparative rétrospective}

PRÉSIDENT DE JURY : $\quad$ Monsieur le Professeur Hervé LEVESQUE

DIRECT.RICE.EUR DE THÈSE : Madame la Docteure Julie GUEUDRY

Monsieur le Docteur Vincent LANGLOIS

MEMBRES DU JURY :

Monsieur le Professeur David SAADOUN

Monsieur le Professeur Olivier VITTECOQ

Monsieur le Docteur Nicolas GIRSZYN 
ANNEE UNIVERSITAIRE 2018 - 2019

U.F.R. SANTÉ DE ROUEN

DOYEN :

Professeur Pierre FREGER

ASSESSEURS :

Professeur Michel GUERBET

Professeur Benoit VEBER

Professeur Guillaume SAVOYE

I - MEDECINE

PROFESSEURS DES UNIVERSITES - PRATICIENS HOSPITALIERS

Mr Frédéric ANSELME
Mme Gisèle APTER
Mme Isabelle AUQUIT AUCKBUR
Mr Jean-Marc BASTE
Mr Fabrice BAUER
Mme Soumeya BEKRI
Mr Ygal BENHAMOU
Mr Jacques BENICHOU
Mr Olivier BOYER
Mme Sophie CANDON
Mr François CARON
Mr Philippe CHASSAGNE
Mr Vincent COMPERE
Mr Jean-Nicolas CORNU
Mr Antoine CUVELIER
Mr Jean-Nicolas DACHER
Mr Stéfan DARMONI
Mr Pierre DECHELOTTE
Mr Stéphane DERREY
Mr Frédéric DI FIORE

$\begin{array}{ll}\text { HCN } & \text { Cardiologie } \\ \text { Havre } & \text { Pédopsychiatrie } \\ \text { HCN } & \text { Chirurgie plastique } \\ \text { HCN } & \text { Chirurgie Thoracique } \\ \text { HCN } & \text { Cardiologie } \\ \text { HCN } & \text { Biochimie et biologie moléculaire } \\ \text { HCN } & \text { Médecine interne } \\ \text { HCN } & \text { Bio statistiques et informatique médicale } \\ \text { UFR } & \text { Immunologie } \\ \text { HCN } & \text { Immunologie } \\ \text { HCN } & \text { Maladies infectieuses et tropicales } \\ \text { HCN } & \text { Médecine interne (gériatrie) } \\ \text { HCN } & \text { Anesthésiologie et réanimation chirurgicale } \\ \text { HCN } & \text { Urologie } \\ \text { HB } & \text { Pneumologie } \\ \text { HCN } & \text { Radiologie et imagerie médicale } \\ \text { HCN } & \text { Informatique médicale et techniques de communication } \\ \text { HCN } & \text { Nutrition } \\ \text { HCN } & \text { Neurochirurgie } \\ \mathrm{CB} & \text { Cancérologie }\end{array}$




\begin{tabular}{|c|c|c|}
\hline Mr Fabien DOGUET & $\mathrm{HCN}$ & Chirurgie Cardio Vasculaire \\
\hline Mr Jean DOUCET & SJ & Thérapeutique - Médecine interne et gériatrie \\
\hline Mr Bernard DUBRAY & $\mathrm{CB}$ & Radiothérapie \\
\hline Mr Frank DUJARDIN & $\mathrm{HCN}$ & Chirurgie orthopédique - Traumatologique \\
\hline Mr Fabrice DUPARC & $\mathrm{HCN}$ & Anatomie - Chirurgie orthopédique et traumatologique \\
\hline Mr Eric DURAND & $\mathrm{HCN}$ & Cardiologie \\
\hline Mr Bertrand DUREUIL & $\mathrm{HCN}$ & Anesthésiologie et réanimation chirurgicale \\
\hline Mme Hélène ELTCHANINOFF & $\mathrm{HCN}$ & Cardiologie \\
\hline Mr Manuel ETIENNE & $\mathrm{HCN}$ & Maladies infectieuses et tropicales \\
\hline Mr Thierry FREBOURG & UFR & Génétique \\
\hline Mr Pierre FREGER & $\mathrm{HCN}$ & Anatomie - Neurochirurgie \\
\hline Mr Jean François GEHANNO & $\mathrm{HCN}$ & Médecine et santé au travail \\
\hline Mr Emmanuel GERARDIN & $\mathrm{HCN}$ & Imagerie médicale \\
\hline Mme Priscille GERARDIN & $\mathrm{HCN}$ & Pédopsychiatrie \\
\hline M. Guillaume GOURCEROL & $\mathrm{HCN}$ & Physiologie \\
\hline Mr Dominique GUERROT & $\mathrm{HCN}$ & Néphrologie \\
\hline Mr Olivier GUILLIN & $\mathrm{HCN}$ & Psychiatrie Adultes \\
\hline Mr Didier HANNEQUIN & $\mathrm{HCN}$ & Neurologie \\
\hline Mr Claude HOUDAYER & $\mathrm{HCN}$ & Génétique \\
\hline Mr Fabrice JARDIN & $\mathrm{CB}$ & Hématologie \\
\hline Mr Luc-Marie JOLY & $\mathrm{HCN}$ & Médecine d'urgence \\
\hline Mr Pascal JOLY & $\mathrm{HCN}$ & Dermato - Vénéréologie \\
\hline Mme Bouchra LAMIA & Havre & Pneumologie \\
\hline Mme Annie LAQUERRIERE & $\mathrm{HCN}$ & Anatomie et cytologie pathologiques \\
\hline Mr Vincent LAUDENBACH & $\mathrm{HCN}$ & Anesthésie et réanimation chirurgicale \\
\hline Mr Joël LECHEVALLIER & $\mathrm{HCN}$ & Chirurgie infantile \\
\hline Mr Hervé LEFEBVRE & $\mathrm{HB}$ & Endocrinologie et maladies métaboliques \\
\hline Mr Thierry LEQUERRE & $\mathrm{HB}$ & Rhumatologie \\
\hline Mme Anne-Marie LEROI & $\mathrm{HCN}$ & Physiologie \\
\hline Mr Hervé LEVESQUE & $\mathrm{HB}$ & Médecine interne \\
\hline Mme Agnès LIARD-ZMUDA & $\mathrm{HCN}$ & Chirurgie Infantile \\
\hline Mr Pierre Yves LITZLER & $\mathrm{HCN}$ & Chirurgie cardiaque \\
\hline Mr Bertrand MACE & $\mathrm{HCN}$ & Histologie, embryologie, cytogénétique \\
\hline M. David MALTETE & $\mathrm{HCN}$ & Neurologie \\
\hline Mr Christophe MARGUET & $\mathrm{HCN}$ & Pédiatrie \\
\hline Mme Isabelle MARIE & $\mathrm{HB}$ & Médecine interne \\
\hline
\end{tabular}




\begin{tabular}{|c|c|c|}
\hline Mr Jean-Paul MARIE & $\mathrm{HCN}$ & Oto-rhino-laryngologie \\
\hline Mr LoÏc MARPEAU & $\mathrm{HCN}$ & Gynécologie - Obstétrique \\
\hline Mr Stéphane MARRET & $\mathrm{HCN}$ & Pédiatrie \\
\hline Mme Véronique MERLE & $\mathrm{HCN}$ & Epidémiologie \\
\hline Mr Pierre MICHEL & $\mathrm{HCN}$ & Hépato-gastro-entérologie \\
\hline M. Benoit MISSET (détachement) & $\mathrm{HCN}$ & Réanimation Médicale \\
\hline Mr Jean-François MUIR (surnombre) & $\mathrm{HB}$ & Pneumologie \\
\hline Mr Marc MURAINE & $\mathrm{HCN}$ & Ophtalmologie \\
\hline Mr Christophe PEILLON & $\mathrm{HCN}$ & Chirurgie générale \\
\hline Mr Christian PFISTER & $\mathrm{HCN}$ & Urologie \\
\hline Mr Jean-Christophe PLANTIER & $\mathrm{HCN}$ & Bactériologie - Virologie \\
\hline Mr Didier PLISSONNIER & $\mathrm{HCN}$ & Chirurgie vasculaire \\
\hline Mr Gaëtan PREVOST & $\mathrm{HCN}$ & Endocrinologie \\
\hline Mr Jean-Christophe RICHARD (détachement) & $\mathrm{HCN}$ & Réanimation médicale - Médecine d'urgence \\
\hline Mr Vincent RICHARD & UFR & Pharmacologie \\
\hline Mme Nathalie RIVES & $\mathrm{HCN}$ & Biologie du développement et de la reproduction \\
\hline Mr Horace ROMAN (disponibilité) & $\mathrm{HCN}$ & Gynécologie - Obstétrique \\
\hline Mr Jean-Christophe SABOURIN & $\mathrm{HCN}$ & Anatomie - Pathologie \\
\hline Mr Guillaume SAVOYE & $\mathrm{HCN}$ & Hépato-gastrologie \\
\hline Mme Céline SAVOYE-COLLET & $\mathrm{HCN}$ & Imagerie médicale \\
\hline Mme Pascale SCHNEIDER & $\mathrm{HCN}$ & Pédiatrie \\
\hline Mr Michel SCOTTE & $\mathrm{HCN}$ & Chirurgie digestive \\
\hline Mme Fabienne TAMION & $\mathrm{HCN}$ & Thérapeutique \\
\hline Mr LUC THIBERVILLE & $\mathrm{HCN}$ & Pneumologie \\
\hline Mr Christian THUILLEZ (surnombre) & $\mathrm{HB}$ & Pharmacologie \\
\hline Mr Hervé TILLY & $\mathrm{CB}$ & Hématologie et transfusion \\
\hline M. Gilles TOURNEL & $\mathrm{HCN}$ & Médecine Légale \\
\hline Mr Olivier TROST & $\mathrm{HCN}$ & Chirurgie Maxillo-Faciale \\
\hline Mr Jean-Jacques TUECH & $\mathrm{HCN}$ & Chirurgie digestive \\
\hline Mr Jean-Pierre VANNIER (surnombre) & $\mathrm{HCN}$ & Pédiatrie génétique \\
\hline Mr Benoît VEBER & $\mathrm{HCN}$ & Anesthésiologie - Réanimation chirurgicale \\
\hline Mr Pierre VERA & $\mathrm{CB}$ & Biophysique et traitement de l'image \\
\hline Mr Eric VERIN & $\mathrm{HB}$ & Service Santé Réadaptation \\
\hline Mr Eric VERSPYCK & $\mathrm{HCN}$ & Gynécologie obstétrique \\
\hline Mr Olivier VITTECOQ & $\mathrm{HB}$ & Rhumatologie \\
\hline Mme Marie-Laure WELTER & $\mathrm{HCN}$ & Physiologie \\
\hline
\end{tabular}


MAITRES DE CONFERENCES DES UNIVERSITES - PRATICIENS HOSPITALIERS

$\begin{array}{lll}\text { Mme Noëlle BARBIER-FREBOURG } & \text { HCN } & \text { Bactériologie - Virologie } \\ \text { Mme Carole BRASSE LAGNEL } & \text { HCN } & \text { Biochimie } \\ \text { Mme Valérie BRIDOUX HUYBRECHTS } & \text { HCN } & \text { Chirurgie Vasculaire } \\ \text { Mr Gérard BUCHONNET } & \text { HCN } & \text { Hématologie } \\ \text { Mme Mireille CASTANET } & \text { HCN } & \text { Pédiatrie } \\ \text { Mme Nathalie CHASTAN } & \text { HCN } & \text { Neurophysiologie } \\ \text { Mme Sophie CLAEYSSENS } & \text { HCN } & \text { Biochimie et biologie moléculaire } \\ \text { Mr Moïse COEFFIER } & \text { HCN } & \text { Nutrition } \\ \text { Mr Serge JACQUOT } & \text { UFR } & \text { Immunologie } \\ \text { Mr Joël LADNER } & \text { HCN } & \text { Epidémiologie, économie de la santé } \\ \text { Mr Jean-Baptiste LATOUCHE } & \text { UFR } & \text { Biologie cellulaire } \\ \text { Mr Thomas MOUREZ (détachement) } & \text { HCN } & \text { Virologie } \\ \text { Mr Gaël NICOLAS } & \text { HCN } & \text { Génétique } \\ \text { Mme Muriel QUILLARD } & \text { HCN } & \text { Biochimie et biologie moléculaire } \\ \text { Mme Laëtitia ROLLIN } & \text { HCN } & \text { Médecine du Travail } \\ \text { Mr Mathieu SALAUN } & \text { HCN } & \text { Pneumologie } \\ \text { Mme Pascale SAUGIER-VEBER } & \text { HCN } & \text { Génétique } \\ \text { Mme Anne-Claire TOBENAS-DUJARDIN } & \text { HCN } & \text { Anatomie } \\ \text { Mr David WALLON } & \text { HCN } & \text { Neurologie } \\ \text { Mr Julien WILS } & \text { HCN } & \text { Pharmacologie }\end{array}$

\section{PROFESSEUR AGREGE OU CERTIFIE}

Mr Thierry WABLE

Mme Mélanie AUVRAY-HAMEL
UFR Communication

UFR Anglais 


\section{PROFESSEURS}

Mr Thierry BESSON

Mr Roland CAPRON (PU-PH)

Mr Jean COSTENTIN (Professeur émérite)

Mme Isabelle DUBUS

Mr François ESTOUR

Mr Loïc FAVENNEC (PU-PH)

Mr Jean Pierre GOULLE (Professeur émérite)

Mr Michel GUERBET

Mme Isabelle LEROUX - NICOLLET

Mme Christelle MONTEIL

Mme Martine PESTEL-CARON (PU-PH)

Mr Rémi VARIN (PU-PH)

Mr Jean-Marie VAUGEOIS

Mr Philippe VERITE

\section{MAITRES DE CONFERENCES}

Mme Cécile BARBOT

Mr Jérémy BELLIEN (MCU-PH)

Mr Frédéric BOUNOURE

Mr Abdeslam CHAGRAOUI

Mme Camille CHARBONNIER (LE CLEZIO)

Mme Elizabeth CHOSSON

Mme Marie Catherine CONCE-CHEMTOB

Mme Cécile CORBIERE

Mr Eric DITTMAR

Mme Nathalie DOURMAP

Mme Isabelle DUBUC

Mme Dominique DUTERTE- BOUCHER

Mr Abdelhakim ELOMRI
Chimie Thérapeutique

Biophysique

Pharmacologie

Biochimie

Chimie Organique

Parasitologie

Toxicologie

Toxicologie

Physiologie

Toxicologie

Microbiologie

Pharmacie clinique

Pharmacologie

Chimie analytique

Chimie Générale et Minérale

Pharmacologie

Pharmacie Galénique

Physiologie

Statistiques

Botanique

Législation pharmaceutique et économie de la santé

Biochimie

Biophysique

Pharmacologie

Pharmacologie

Pharmacologie

Pharmacognosie 
Mr Gilles GARGALA (MCU-PH)

Mme Nejla EL GHARBI-HAMZA

Mme Marie-Laure GROULT

Mr Hervé HUE

Mme Laetitia LE GOFF

Mme Hong LU

M. Jérémie MARTINET (MCU-PH)

Mme Marine MALLETER

Mme Sabine MENAGER

Mme Tiphaine ROGEZ-FLORENT

Mr Mohamed SKIBA

Mme Malika SKIBA

Mme Christine THARASSE

Mr Frédéric ZIEGLER

\section{PROFESSEURS ASSOCIES}

Mme Cécile GUERARD-DETUNCQ

Mr Jean-François HOUIVET

PROFESSEUR CERTIFIE

Mme Mathilde GUERIN

ASSISTANT HOSPITALO-UNIVERSITAIRE

Mme Anaïs SOARES
Parasitologie

Chimie analytique

Botanique

Biophysique et mathématiques

Parasitologie - Immunologie

Biologie

Immunologie

Toxicologie

Chimie organique

Chimie analytique

Pharmacie galénique

Pharmacie galénique

Chimie thérapeutique

Biochimie

Pharmacie officinale

Pharmacie officinale

Anglais

Bactériologie

\section{ATTACHES TEMPORAIRES D'ENSEIGNEMENT ET DE RECHERCHE}

Mme Sophie MOHAMED

Chimie Organique 
Mme Cécile BARBOT

Mr Thierry BESSON

Mr Roland CAPRON

Mme Marie-Catherine CONCE-CHEMTOB

Mme Elisabeth CHOSSON

Mme Isabelle DUBUS

Mr Abdelhakim ELOMRI

Mr Loïc FAVENNEC

Mr Michel GUERBET

Mr François ESTOUR

Mme Isabelle LEROUX-NICOLLET

Mme Martine PESTEL-CARON

Mr Mohamed SKIBA

Mr Rémi VARIN

M. Jean-Marie VAUGEOIS

Mr Philippe VERITE
Chimie Générale et minérale

Chimie thérapeutique

Biophysique

Législation et économie de la santé

Botanique

Biochimie

Pharmacognosie

Parasitologie

Toxicologie

Chimie organique

Physiologie

Microbiologie

Pharmacie galénique

Pharmacie clinique

Pharmacologie

Chimie analytique 
PROFESSEUR MEDECINE GENERALE

Mr Jean-Loup HERMIL (PU-MG) UFR Médecine générale

\section{MAITRE DE CONFERENCE MEDECINE GENERALE}

Mr Matthieu SCHUERS (MCU-MG) UFR Médecine générale

PROFESSEURS ASSOCIES A MI-TEMPS - MEDECINS GENERALISTE

$\begin{array}{lcc}\text { Mme Laëtitia BOURDON } & \text { UFR } & \text { Médecine Générale } \\ \text { Mr Emmanuel LEFEBVRE } & \text { UFR } & \text { Médecine Générale } \\ \text { Mme Elisabeth MAUVIARD } & \text { UFR } & \text { Médecine générale } \\ \text { Mr Philippe NGUYEN THANH } & \text { UFR } & \text { Médecine générale } \\ \text { Mme Marie Thérèse THUEUX } & \text { UFR } & \text { Médecine générale }\end{array}$

MAITRE DE CONFERENCES ASSOCIE A MI-TEMPS - MEDECINS GENERALISTES

$\begin{array}{lcl}\text { Mr Pascal BOULET } & \text { UFR } & \text { Médecine générale } \\ \text { Mr Emmanuel HAZARD } & \text { UFR } & \text { Médecine Générale } \\ \text { Mme Marianne LAINE } & \text { UFR } & \text { Médecine Générale } \\ \text { Mme Lucile PELLERIN } & \text { UFR } & \text { Médecine générale } \\ \text { Mme Yveline SEVRIN } & \text { UFR } & \text { Médecine générale }\end{array}$




\section{ENSEIGNANTS MONO-APPARTENANTS}

\section{PROFESSEURS}

Mr Serguei FETISSOV (med)

Mr Paul MULDER (phar)

Mme Su RUAN (med)

\section{MAITRES DE CONFERENCES}

Mr Sahil ADRIOUCH (med)

Mme Gaëlle BOUGEARD-DENOYELLE (med)

Mme Carine CLEREN (med)

M. Sylvain FRAINEAU (med)

Mme Pascaline GAILDRAT (med)

Mr Nicolas GUEROUT (med)

Mme Rachel LETELLIER (med)

Mme Christine RONDANINO (med)

Mr Antoine OUVRARD-PASCAUD (med)

Mr Frédéric PASQUET

Mr Youssan Var TAN

Mme Isabelle TOURNIER (med)
Physiologie (ADEN)

Sciences du Médicament

Génie Informatique
Biochimie et biologie moléculaire (Unité Inserm 905)

Biochimie et biologie moléculaire (UMR 1079)

Neurosciences (Néovasc)

Physiologie (Inserm U 1096)

Génétique moléculaire humaine (UMR 1079)

Chirurgie Expérimentale

Physiologie

Physiologie de la reproduction

Physiologie (Unité Inserm 1076)

Sciences du langage, orthophonie

Immunologie

Biochimie (UMR 1079)

\section{CHEF DES SERVICES ADMINISTRATIFS : Mme Véronique DELAFONTAINE}

HCN - Hôpital Charles Nicolle

CB - Centre Henri Becquerel

CRMPR - Centre Régional de Médecine Physique et de Réadaptation
HB - Hôpital de BOIS GUILLAUME

CHS - Centre Hospitalier Spécialisé du Rouvray

SJ - Saint Julien Rouen 
Par délibération en date du 3 mars 1967, la faculté a arrêté que les opinions émises dans les dissertations qui lui sont présentées doivent être considérées comme propres à leurs auteurs et qu'elle n'entend leur donner aucune approbation ni improbation. 


\section{Table des matières}

Liste des abréviations

I. INTRODUCTION : LES UVEITES NON-INFECTIEUSES : DE LA DEFINITION ANATOMO-CLINIQUE A LA PRISE EN CHARGE THERAPEUTIQUE .................................................................... 3

1.1. Définition des uvéites : classification anatomique et chronologique ................................ 3

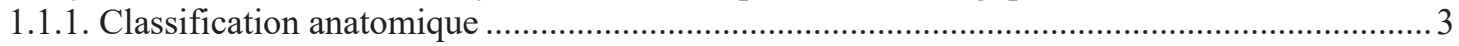

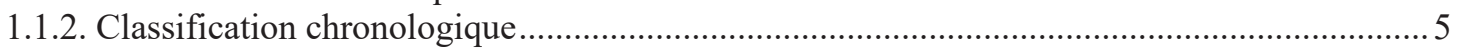

1.2. Sémiologie inflammatoire ophtalmologique ............................................................. 7

1.2.1. Classification de l'activité de l'uvéite ...................................................................... 7

1.2.2. Sémiologie oculaire d'orientation étiologique .............................................................. 9

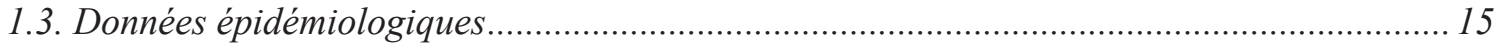

1.4. Les uvéites non-infectieuses : entité clinique aux nombreux diagnostics étiologiques......... 17

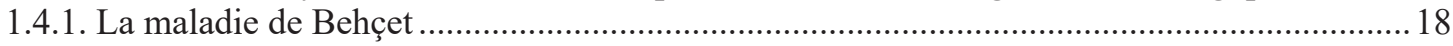

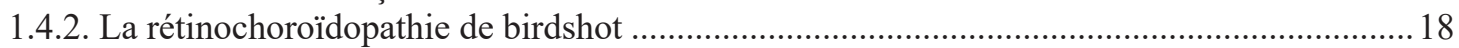

1.4.3. La choroïdite multifocale idiopathique ...................................................................... 19

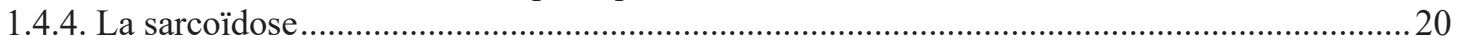

1.4.5. La maladie de Vogt-Koyanagi-Harada ..............................................................................2 21

1.5. Les uvéites non-infectieuses : de la physiopathologie à la prise en charge thérapeutique ... 22

1.6. Les uvéites non-infectieuses : des stratégies thérapeutiques en pleine évolution.................25

1.6.1. Le choix actuel de première intention, en cas d'intensification thérapeutique : les immunosuppresseurs conventionnels .................................................................................26

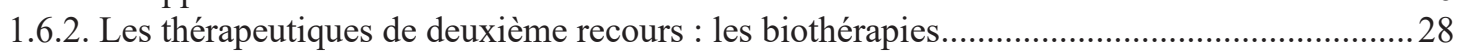

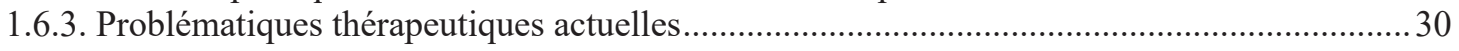

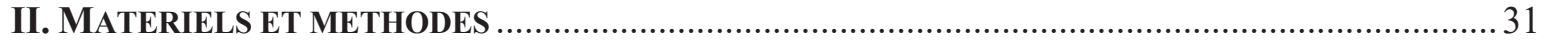

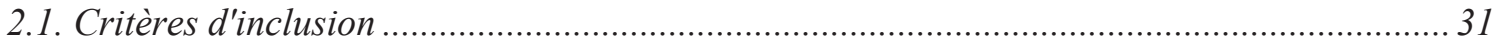

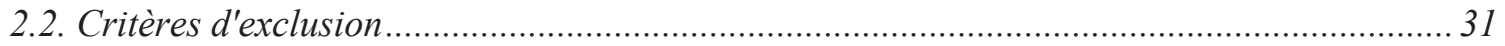

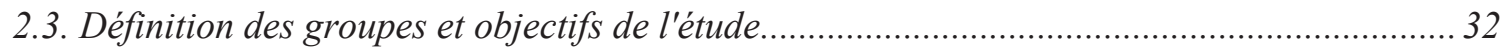

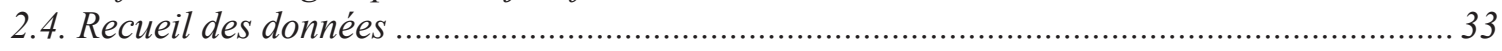

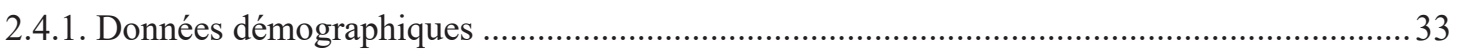

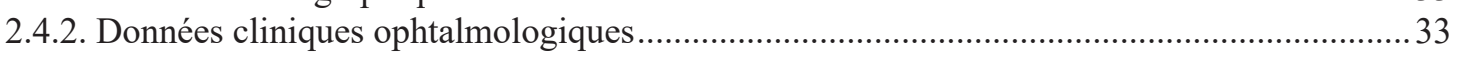

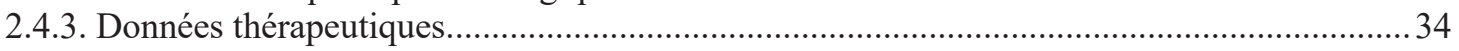

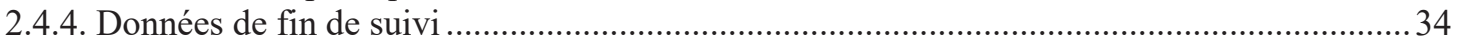

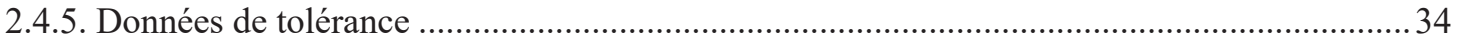

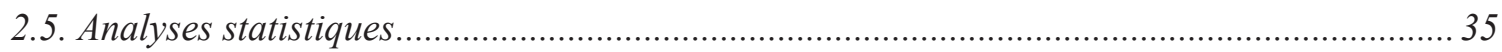

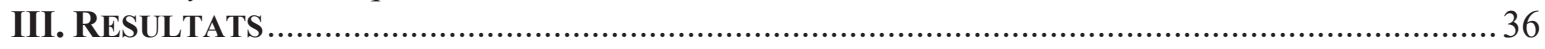

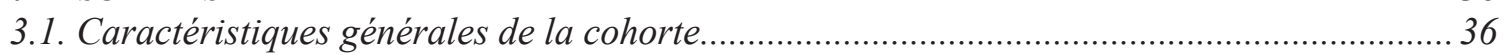

3.2. Description du groupe biothérapie ............................................................... 38

3.2.1. Caractéristiques générales et ophtalmologiques lors de l'introduction du traitement ............38

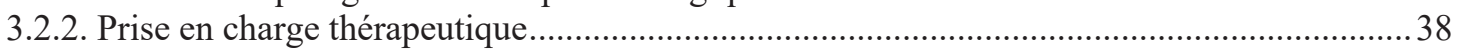

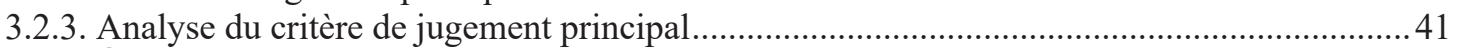

3.2.4. Évolution des paramètres ophtalmologiques sous traitement ......................................... 41

3.2.5. Caractéristiques à la fin du suivi ............................................................................ 45

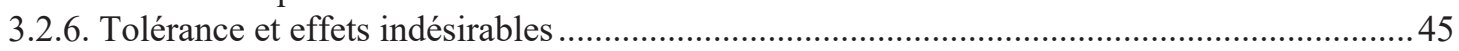

3.2.7. Analyse de sous-groupes selon le type de biothérapie reçu ................................................46

3.3. Description du groupe immunosuppresseur conventionnel ..............................................4 48

3.3.1. Caractéristiques générales et ophtalmologiques lors de l'introduction du traitement ............48

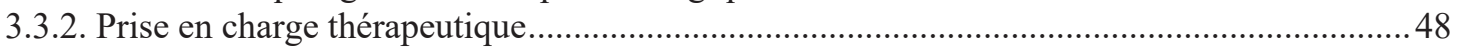

3.3.3. Analyse du critère de jugement principal.............................................................5

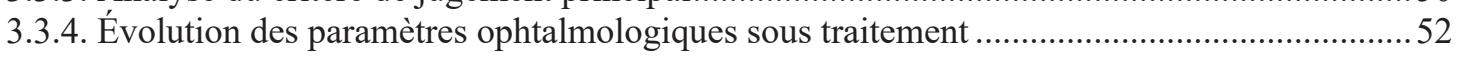

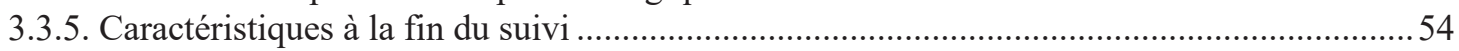

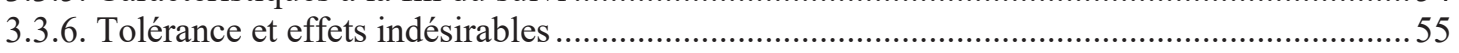

3.3.7. Analyse de sous-groupes selon le type d'immunosuppresseur conventionnel reçu ...............55

3.4. Comparaison des deux stratégies thérapeutiques .........................................................5.5.

3.4.1. Caractéristiques générales et ophtalmologiques lors de l'introduction du traitement ............58

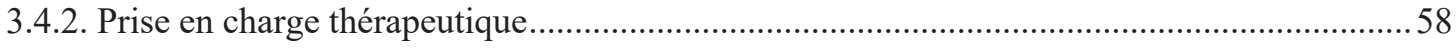

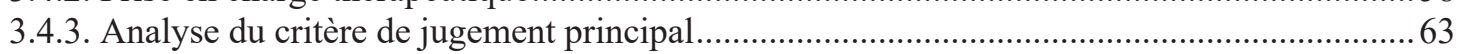


3.4.4. Évolution des paramètres ophtalmologiques sous traitement

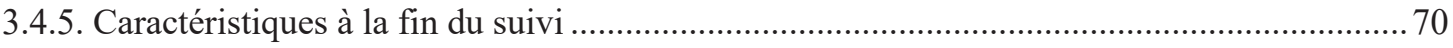

3.5. Analyse de sous-groupes selon le type d'étiologie ................................................................ 72

3.6. Facteurs associés à la prescription de biothérapie ............................................................... 75

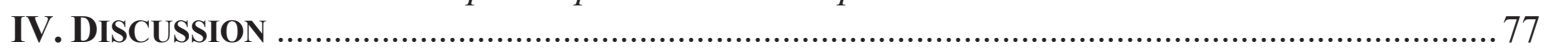

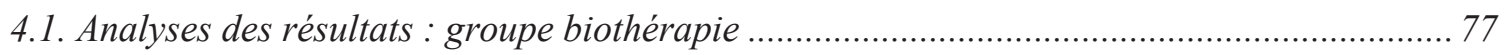

4.2. Analyses des résultats : groupe immunosuppresseur conventionnel .................................. 83

4.3. Comparaison des deux stratégies thérapeutiques ............................................................ 86

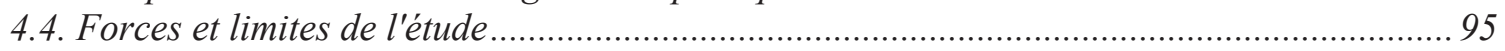

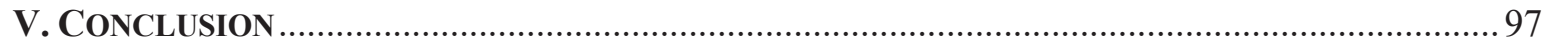

Annexe 1 : Liste des codages CIM10 ayant permis l'extraction des données des patients suivis en

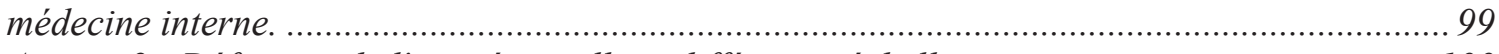

Annexe 2 : Définition de l'acuité visuelle et différentes échelles............................................. 100 Annexe 3 : E-poster présenté en section orale commentée le vendredi 30 aout 2019 au Congrès

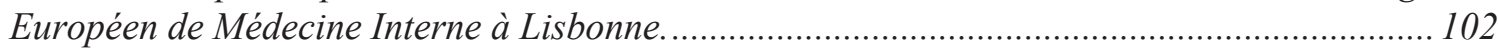

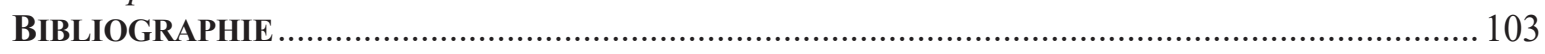




\section{Liste des abréviations}

$\begin{array}{ll}\text { ADN } & \text { Acide désoxyribonucléique } \\ \text { Ag-S } & \text { Antigène soluble rétinien } \\ \text { AMM } & \text { Autorisation de mise sur le marché } \\ \text { CD } & \text { Cluster de différenciation } \\ \text { EAU } & \text { Uvéite auto-immune expérimentale } \\ \text { ETDRS } & \text { Early treatment diabetic retinopathy study chart } \\ \text { EULAR } & \text { European league against rheumatism } \\ \text { HLA } & \text { Human leukocyte antigen } \\ \text { HSV } & \text { Herpès simplex virus } \\ \text { ICBD } & \text { International criteria for Behçet's disease } \\ \text { ICER } & \text { Ratio coût-efficacité incrémentiel } \\ \text { IL } & \text { Interleukine } \\ \text { IQR } & \text { Interquartile range } \\ \text { IRBP } & \text { Interphotoreceptor retinoid binding protein } \\ \text { ISG } & \text { International study group } \\ \text { IUSG } & \text { International uveitis study group } \\ \text { KO } & \text { Knock out } \\ \text { IWOS } & \text { International workshop on ocular sarcoidosis } \\ \text { MAR } & \text { Minimum angle of resolution } \\ \text { MUST } & \text { Multicenter uveitis steroid treatment } \\ \text { NFAT } & \text { Nuclear factor of activated T-cells } \\ \text { OCT } & \text { Tomographie par cohérence optique } \\ \text { OMS } & \text { Organisation mondiale de la santé } \\ \text { QUALY } & \text { Année de vie ajustée sur la qualité } \\ \text { SITE } & \text { Systemic immunosuppressive therapy for eye diseases } \\ \text { SUN } & \text { Standardization of uveitis nomenclature working group } \\ \text { TCR } & \text { T-cell receptor } \\ \text { TGF } & \text { Transforming growth factor } \\ \text { Th } & \text { Lymphocyte T helper } \\ \text { TLR } & \text { Toll-like receptor } \\ \text { TNF } & \text { Tumor necrosis factor } \\ \text { VEGF } & \text { Vascular endothelial growth factor } \\ \text { VZV } & \text { Varicella zoster virus } \\ & \end{array}$




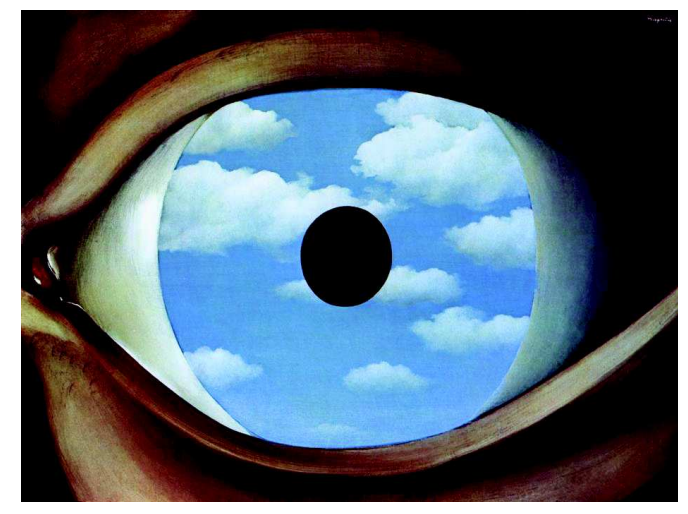

René MAGRITTE, Le faux miroir, 1929. 


\section{Introduction : les uvéites non-infectieuses : de la définition anatomo- clinique à la prise en charge thérapeutique}

\subsection{Définition des uvéites : classification anatomique et chronologique}

\subsubsection{Classification anatomique}

Les uvéites s'intègrent dans un cadre nosologique vaste, définies à la fois de manière anatomique, sémiologique et étiologique. Si historiquement la première classification revient au Docteur Geraint James en 1964 (1), il aura fallu attendre la création d'un groupe international de travail sur les uvéites en 1978, International uveitis study group (IUSG), pour aboutir à une nouvelle classification en 1987. Cette classification a entrepris de définir les uvéites selon leur localisation anatomique, mais aussi leur évolution (2). En 2005, le Standardization of uveitis nomenclature working group (SUN), comprenant certains membres de l'IUSG, a affiné ce travail de standardisation sur la nomenclature des uvéites, pour obtenir une description homogène internationale (3).

L'uvée correspond à la membrane intermédiaire du bulbe oculaire, et est constituée de la choroïde, tissu vasculaire nutritif, des corps ciliaires et de l'iris (Figure 1) (4). Cependant, le terme d'uvéite s'intègre dans un registre plus étendu qu'une inflammation isolée de l'uvée, englobant ainsi les atteintes inflammatoires de la rétine et ses vaisseaux, et de la papille.

Cliniquement, les patients peuvent présenter, en fonction du segment oculaire atteint, un œil rouge et/ou douloureux, une baisse d'acuité visuelle plus ou moins brutale et profonde ou encore un flou visuel (5).

Le SUN a défini une classification anatomique basée sur la localisation intraoculaire segmentaire de l'inflammation. Elle repose sur la localisation initiale de l'inflammation, sans tenir compte du siège éventuel des complications (Tableau 1). Les uvéites unilatérales doivent faire éliminer une cause infectieuse en première intention. A l'inverse, devant une uvéite bilatérale, le bilan étiologique sera d'emblée orienté vers une cause inflammatoire systémique, ou cantonnée à l'œil.

Les uvéites antérieures sont caractérisées par une atteinte de l'iris et du corps ciliaire (Figure 2) (6). Il s'agit de la localisation la plus fréquente des uvéites, représentant 50 à $60 \%$ des diagnostics d'uvéites en centre tertiaire (7). Dans 8 à $12 \%$ des cas, cette uvéite antérieure s'intègre dans le cadre étiologique d'une spondylarthropathie. 


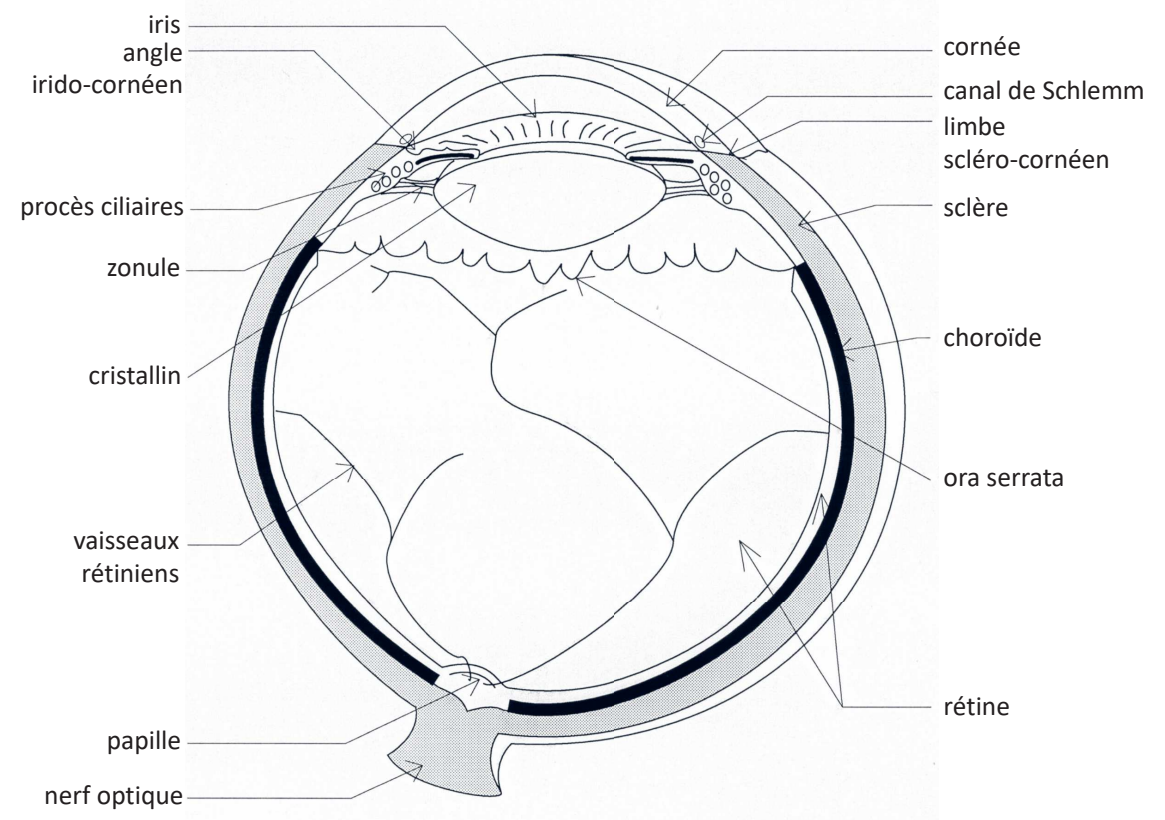

Figure 1 : Représentation schématique du bulbe oculaire [issue du polycopié national du collège des ophtalmologistes universitaires de France (4)].
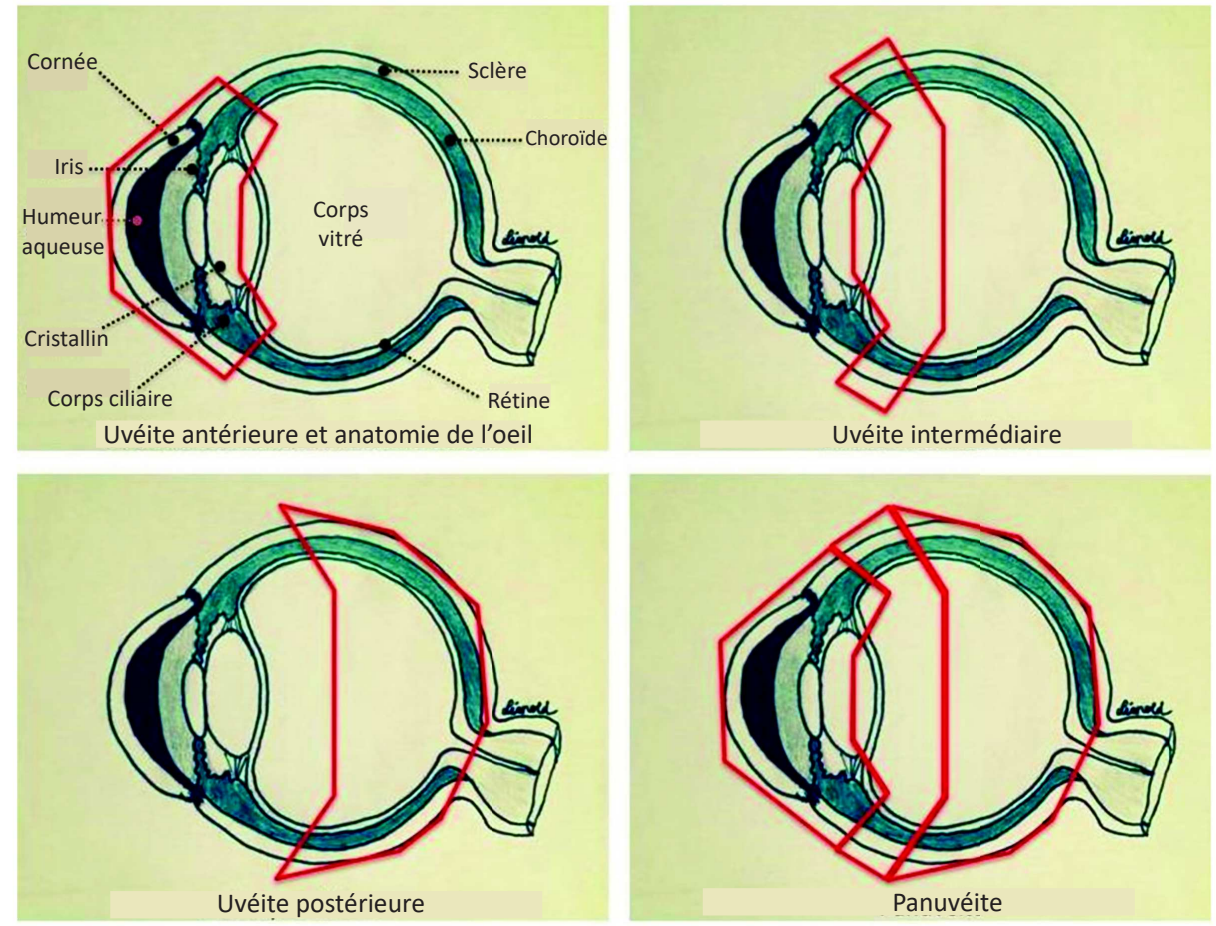

Figure 2 : Représentation schématique des uvéites en fonction de leur localisation [issue de Sève et $a l$. Rev Med Interne, 2018 (8)]. 
Ces uvéites répondent favorablement aux traitements topiques locaux du fait de leur localisation anatomique, et ne vont pas nécessiter, en l'absence de complication, de traitement systémique.

Les uvéites intermédiaires sont caractérisées par une localisation principale de l'inflammation au niveau de la choriorétine périphérique (3). La présence d'engainements vasculaires périphériques ou d'œdème maculaire ne remet pas en cause cette classification. Il s'agit de la forme la moins fréquente, représentant uniquement $15 \%$ des cas d'uvéites (7). Dans plus de $50 \%$ des cas, il s'agit d'uvéites idiopathiques.

Les uvéites postérieures, caractérisées par une atteinte rétinienne et/ou choroïdienne, représente 15 à $30 \%$ des cas d'uvéites, d'origine infectieuse majoritairement (7).

Enfin, les panuvéites affichent une prévalence plus disparate, allant de $7 \%$ à $40 \%$ des cas d'uvéites $(9,10)$. Cette variabilité est secondaire aux biais de recrutement des différents centres. Il s'agit des cas où l'inflammation n'a pas de segment oculaire prédominant. L'inflammation doit être retrouvée au niveau du segment antérieur, du vitré, de la rétine et/ou de la choroïde (3). Les panvéites sont la localisation la plus fréquente des uvéites au cours de la maladie de Behçet.

Outre son intérêt nosologique permettant une concordance des descriptions lors des études internationales, cette définition anatomique a également pour but d'orienter le diagnostic étiologique. A titre d'exemple, les uvéites intermédiaires doivent orienter le diagnostic vers une sarcoïdose ou une sclérose en plaques. Devant une uvéite antérieure, on recherchera en premier lieu une spondylarthropathie. Certains diagnostics sont cantonnés à un segment oculaire, telle la maladie de birdshot touchant uniquement le segment postérieur ou à l'inverse, la sarcoïdose pouvant touchant l'ensemble des segments $(1,5)$.

\subsubsection{Classification chronologique}

Le travail de l'équipe du SUN a permis également de définir le caractère aigu ou chronique de l'uvéite (3). Une uvéite aiguë correspond à un épisode inflammatoire brutal d'une durée inférieure à 3 mois (Tableau 2). Les uvéites récidivantes ou récurrentes se définissent comme des épisodes inflammatoires répétés, séparés par des périodes d'inactivité sans traitement de plus de 3 mois. A l'inverse, les uvéites chroniques sont en lien avec des récidives inflammatoires précoces, survenant dans un délai inférieur à 3 mois après arrêt du traitement spécifique (1), responsables d'une inflammation prolongée. 


\begin{tabular}{|l|l|l|}
\hline \multicolumn{1}{|c|}{ Type } & \multicolumn{1}{|c|}{ Site initial de l'inflammation } & \multicolumn{1}{c|}{ Inclus } \\
\hline Antérieure & Chambre antérieure & $\begin{array}{l}\text { Iris } \\
\text { Iridocyclite } \\
\text { Cyclite antérieure }\end{array}$ \\
\hline Intermédiaire & Vitré & $\begin{array}{l}\text { Pars planite } \\
\text { Hyalite } \\
\text { Cyclite postérieure }\end{array}$ \\
\hline Postérieure & Rétine ou choroïde & $\begin{array}{l}\text { Choroïdite focale, multifocale, diffuse } \\
\text { Choriorétinite } \\
\text { Rétinochoroïdite } \\
\text { Rétinite } \\
\text { Neurorétinite }\end{array}$ \\
\hline Panuvéite & $\begin{array}{l}\text { Chambre antérieure,vitré, rétine } \\
\text { et choroïde }\end{array}$ & Toutes \\
\hline
\end{tabular}

Tableau 1 : Classification anatomique des uvéites [d'après Jabs et al. Am J Opthalmol, 2005(3)].

\begin{tabular}{|l|l|l|}
\hline \multicolumn{1}{|c|}{ Catégorie } & \multicolumn{1}{|c|}{ Description } & \multicolumn{1}{c|}{ Commentaire } \\
\hline Evolution & Aiguë & $\begin{array}{l}\text { Épisode caractérisé par un déclenchement } \\
\text { soudain mais limité dans le temps (<3 mois) }\end{array}$ \\
\hline & Récurrente & $\begin{array}{l}\text { Épisodes répétés séparés par des périodes } \\
\text { d'inactivité sans traitement d'une durée } \geq 3 \\
\text { mois }\end{array}$ \\
\hline & Chronique & $\begin{array}{l}\text { Uvéite persistante avec rechute moins de 3 } \\
\text { mois après l'arrêt du traitement }\end{array}$ \\
\hline
\end{tabular}

Tableau 2 : Classification selon la durée et l'évolution des uvéites [d'après Jabs et al. Am J Opthalmol, 2005(3)]. 
A nouveau, cette classification chronologique va permettre d'orienter le diagnostic et surtout, le bilan étiologique initial. Celui-ci sera différent devant un épisode unique d'uvéite aiguë ou de multiples poussées inflammatoires. Une uvéite antérieure aiguë brutale est plutôt l'apanage des causes infectieuses ou des uvéites associées à l'haplotype Human leukocyte antigen (HLA) B27. Les uvéites au cours de la sarcoïdose sont plutôt de type chronique (8).

\subsection{Sémiologie inflammatoire ophtalmologique}

\subsubsection{Classification de l'activité de l'uvéite}

L'examen ophtalmologique à la lampe à fente garantit une description et une classification du degré d'inflammation intraoculaire. La lampe à fente est un microscope binoculaire permettant de voir de façon détaillée les différents éléments du segment antérieur. Son système d'éclairage constitué d'une fente permet d'effectuer une coupe optique des différentes structures du segment antérieur (4). L'inflammation sera évaluée au niveau de la chambre antérieure grâce au Tyndall cellulaire et Tyndall protéique, et au niveau du vitré par l'échelle du flou vitréen (11).

A la lampe à fente, il est possible de quantifier de façon semi-quantitative les cellules visibles au niveau de l'humeur aqueuse de la chambre antérieure. Ces cellules proviennent du corps ciliaire ou de l'iris inflammatoires (1). Le Tyndall cellulaire correspond au décompte des cellules en suspension dans l'humeur aqueuse dans un faisceau lumineux de $1 * 1 \mathrm{~mm}$ à $45^{\circ}$ (Tableau 3).

Le Tyndall protéique équivaut à une évaluation des débris cellulaires et protéiques de l'humeur aqueuse dans un faisceau lumineux identique à celui de l'évaluation du Tyndall cellulaire (1). A la différence de ce dernier, le Tyndall protéique repose sur une évaluation subjective de la netteté de visualisation de l'iris et du cristallin (3) (Tableau 4).

L'évaluation de l'inflammation intra-vitréenne repose sur les mêmes principes de détection des dépôts protéiques et cellulaires. Elle s'effectue également au microscope binoculaire, après dilatation. Cependant, la quantification au niveau du vitré est plus délicate à la lampe à fente, car la visualisation du vitré moyen et postérieur peut être gênée par une réaction inflammatoire importante dans la chambre antérieure (1). Les cellules inflammatoires intra-vitréennes proviennent de la choroïde, de la rétine et du corps ciliaire. L'équipe de travail du SUN n'a pas défini d'échelle d'évaluation de l'inflammation intra-vitréenne. L'échelle la plus utilisée actuellement est celle du flou vitréen (Tableau 5), élaborée par Nussenblatt (11), évaluant à la fois les débris cellulaires et protéiques. 


\begin{tabular}{|c|l|}
\hline Grade & \multicolumn{1}{|c|}{$\begin{array}{c}\text { Nombre de cellules en chambre } \\
\text { antérieure }\end{array}$} \\
\hline 0 & $<1$ \\
\hline $0,5+$ & 1 à 5 \\
\hline $1+$ & 6 à 15 \\
\hline $2+$ & 16 à 25 \\
\hline $3+$ & 26 à 50 \\
\hline $4+$ & $>50$ \\
\hline
\end{tabular}

Tableau 3 : Tyndall cellulaire en chambre antérieure [issu de Bodaghi et al. Atlas en ophtalmologie, 2017 (1)].

\begin{tabular}{|c|l|}
\hline Grade & \multicolumn{1}{|c|}{ Description } \\
\hline 0 & Absent \\
\hline $1+$ & Léger \\
\hline $2+$ & Modéré (détails de l'iris et du cristallin clairement visibles) \\
\hline $3+$ & Marqué (détails de l'iris et du cristallin flous) \\
\hline $4+$ & Intense (fibrine) \\
\hline
\end{tabular}

Tableau 4 : Tyndall protéique en chambre antérieure [issu de Bodaghi et al. Atlas en ophtalmologie, 2017 (1)].

\begin{tabular}{|c|l|}
\hline Grade & \multicolumn{1}{c|}{ Description } \\
\hline 0 & Vitré clair \\
\hline 1 & $\begin{array}{l}\text { Présence de cellules ne gênant pas la } \\
\text { visualisation des détails rétiniens }\end{array}$ \\
\hline 2 & Détails rétiniens mal visibles \\
\hline 3 & Fond d'œil mal visible excepté les gros vaisseaux \\
\hline 4 & $\begin{array}{l}\text { Opacités vitréennes denses. } \\
\text { Fond d'œil non visible }\end{array}$ \\
\hline
\end{tabular}

Tableau 5 : Échelle de flou vitréen [issu de Bodaghi et al. Atlas en ophtalmologie, 2017 (1)]. 
Ces différents éléments sémiologiques ont ainsi permis de déterminer l'activité de l'uvéite en fonction de l'évolution de l'inflammation intraoculaire au cours du temps (Tableau 6). Cette nomenclature, définie par le SUN, a pour finalité d'homogénéiser les définitions d'amélioration ou d'aggravation lors des essais thérapeutiques (3).

Ainsi, une aggravation de l'uvéite correspond à une augmentation de deux niveaux de degré d'inflammation de l'humeur aqueuse (Tyndall protéique) ou du vitré (échelle du flou vitréen) ou d'une augmentation du degré 3+ au degré maximal 4+. Une amélioration de l'uvéite se définie, à l'inverse, comme une diminution de deux niveaux de degré d'inflammation de l'humeur aqueuse ou du vitré ou d'une diminution du degré $1+$ à 0 .

Une uvéite inactive équivaut à l'absence d'activité inflammatoire intraoculaire avec un grade de Tyndall protéique et d'échelle du flou vitréen évalués à 0 . Enfin, une rémission clinique correspond à la situation d'inactivité inflammatoire intraoculaire depuis plus de 3 mois après arrêt du traitement (3).

\subsubsection{Sémiologie oculaire d'orientation étiologique}

D'autres éléments de sémiologie ophtalmologique, n'ayant pas pour vertu d'évaluer l'activité inflammatoire intraoculaire, vont néanmoins permettre une orientation diagnostique étiologique.

Les précipités rétro-cornéens (Figure 3) correspondent à des dépôts de cellules inflammatoires sur l'endothélium cornéen (1). Ces cellules peuvent être des polynucléaires neutrophiles, les précipités apparaissant alors fins, ou des macrophages, les précipités étant alors plus visibles et prenant le nom de précipités en graisse de mouton ou granulomateux. La couleur de ces précipités varie en fonction de leur ancienneté : blanchâtre pour les lésions récentes, alors que les lésions évoluant depuis plus longtemps apparaissent, le plus souvent, pigmentées. D'autres éléments sémiologiques permettent également de poser le diagnostic d'uvéite granulomateuse. Il s'agit de la présence, au niveau de l'iris, de nodules de Köeppe (localisés au niveau du sphincter irien) (Figure 4) ou de nodules de Busacca (localisés au niveau de la collerette irienne) (1). Ces nodules sont également composés d'agrégats de cellules inflammatoires. Cet adjectif de granulomateux décrit uniquement l'aspect visuel, sans support histologique. Cet élément sémiologique est suffisant pour exclure certains diagnostics. 


\begin{tabular}{|l|l|}
\hline \multicolumn{1}{|c|}{ Catégorie } & \multicolumn{1}{c|}{ Définition } \\
\hline Inactive & Grade 0 (Tyndall protéique et flou vitréen) \\
\hline Aggravation & $\begin{array}{l}\text { Augmentation de 2 niveaux de grade d'inflammation (Tyndall } \\
\text { protéique ou flou vitréen) } \\
\text { Ou augmentation au grade maximal (4+) }\end{array}$ \\
\hline Amélioration & $\begin{array}{l}\text { Diminution de 2 niveaux de grade d'inflammation (Tyndall } \\
\text { protéique ou flou vitréen) } \\
\text { Ou diminution jusqu'au grade d'inactivité (0) }\end{array}$ \\
\hline Rémission & Inactivité depuis plus de 3 mois après arrêt du traitement \\
\hline
\end{tabular}

Tableau 6 : Classification de l'activité de l'uvéite [d'après Jabs et al. Am J Opthalmol, 2005(3)].

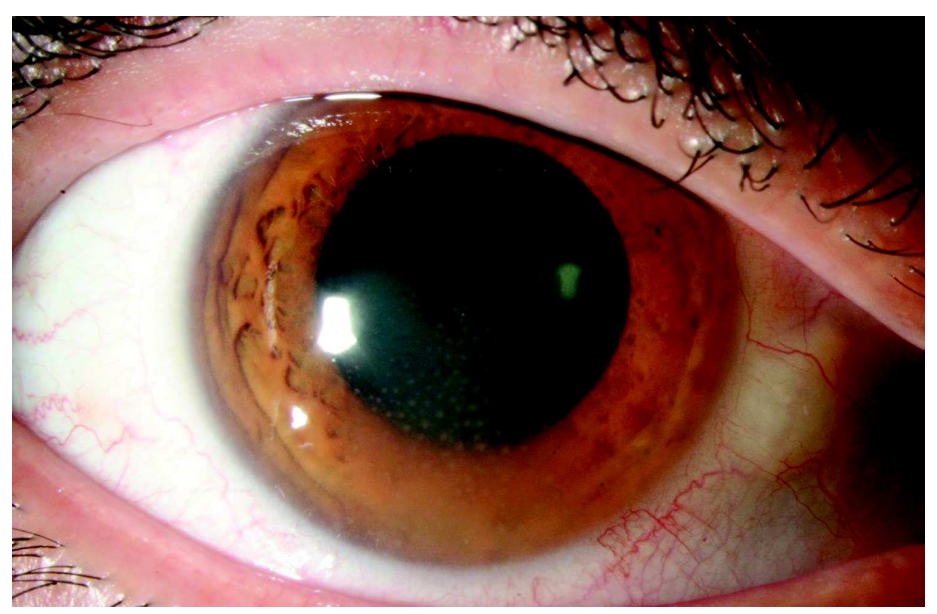

Figure 3 : Précipités rétro-cornéens (issue de la bibliothèque d'images du service d'ophtalmologie de Rouen).

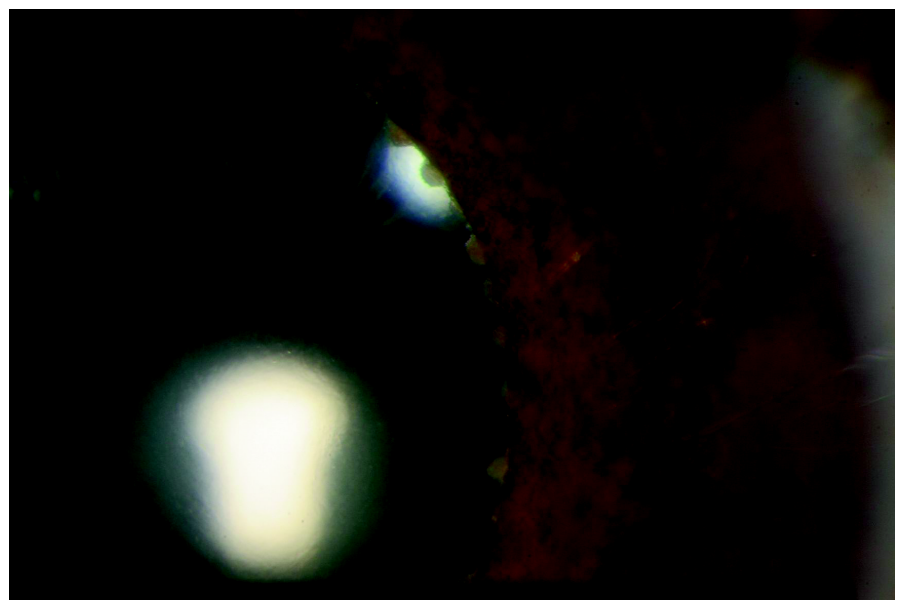

Figure 4 : Nodules de Köeppe (issue de la bibliothèque d'images du service d'ophtalmologie de Rouen). 
En effet, les uvéites associées à la maladie de Behçet ou à l'haplotype HLA B27 ne sont jamais de type granulomateuses, en dehors de situations post-opératoires (6). A l'inverse, certaines uvéites peuvent être de type granulomateuses, telles la sarcoïdose ou la maladie de VogtKoyanagi-Harada. Cependant, l'absence de visualisation de précipités rétro-cornéens en graisse de mouton ou de nodules iriens ne permet pas d'exclure ces diagnostics.

Les synéchies (Figure 5) apparaissent comme une adhérence inflammatoire entre la face postérieure de l'iris et la capsule antérieure du cristallin (4). La présence de synéchies iriennes rend peu probable les diagnostics de rétinochoroïdopathie de birdshot ou de choroïdite multifocale (1).

La présence d'un hypopion (Figure 6), correspondant à la sédimentation dans la partie inférieure de la chambre antérieure de cellules inflammatoires, va orienter vers les diagnostics de maladie de Behçet ou d'uvéite associée à l'haplotype HLA B27. A l'inverse, les uvéites granulomateuses se présentent rarement sous la forme d'un hypopion, même si toute uvéite sévère peut être associée à cet élément sémiologique. La présence d'un hypopion doit, avant tout, faire éliminer le diagnostic différentiel d'endophtalmie.

La mesure de la pression intraoculaire permet de définir les uvéites hypertensives (pression intraoculaire $>22$ millimètres de mercure). Cette hypertonie est généralement secondaire à une inflammation du trabéculum. Les principales étiologies à évoquer sont alors les uvéites infectieuses et la sarcoïdose (1).

Enfin, l'étude du fond d'œil va permettre de distinguer les atteintes inflammatoires de la choroïde ou de la rétine, ainsi que leurs localisations périphérique ou maculaire, sans omettre l'examen de la papille optique (1). Un œdème papillaire (bords de la papille apparaissant alors flous) va faire rechercher une neuropathie optique associée à l'uvéite, observée lors de la maladie de Behçet ou la sarcoïdose. L'analyse du réseau vasculaire rétinien s'effectue lors du fond d'œil, mais également à travers l'angiographie à la fluorescéine, assurant une étude dynamique du réseau vasculaire. L'atteinte inflammatoire des parois vasculaires porte le nom de vascularite rétinienne, sans pour autant revêtir la définition histologique des vascularites. Cette atteinte inflammatoire apparaît comme une imprégnation progressive de la paroi vasculaire par la fluorescéine (staining) puis une diffusion de ce colorant (leakage) dans l'espace périvasculaire (Figure 7) (1). 


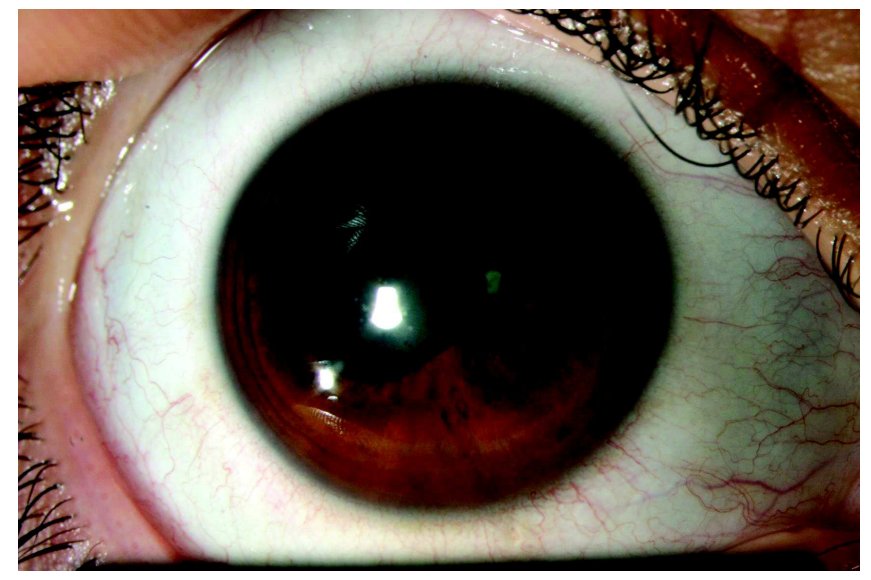

Figure 5 : Synéchies irido-cristalliniennes (issue de la bibliothèque d'images du service d'ophtalmologie de Rouen).

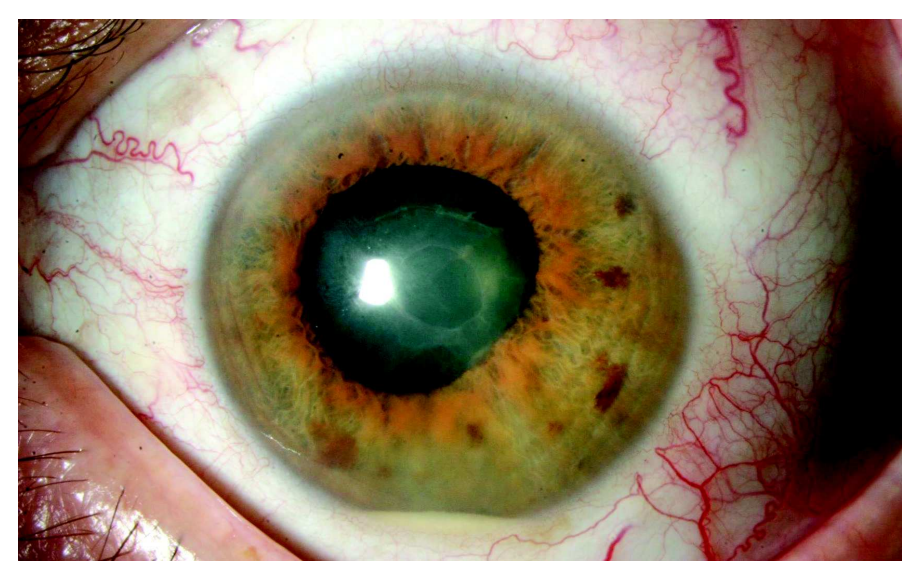

Figure 6 : Hypopion au cours d'une uvéite (issue de la bibliothèque d'images du service d'ophtalmologie de Rouen).

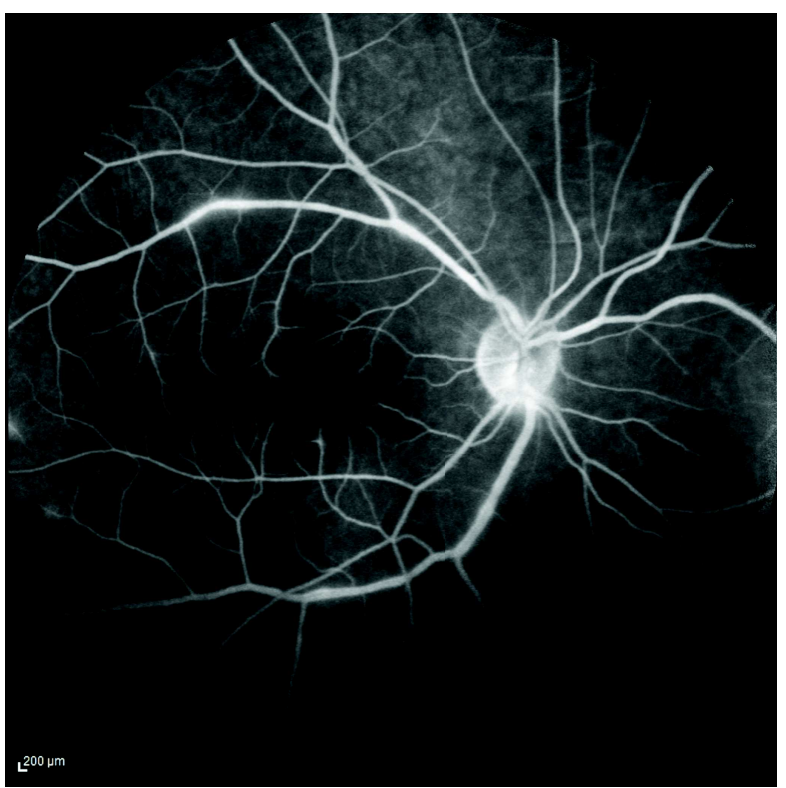

Figure 7 : Angiographie à la fluorescéine. Aspect irrégulier du calibre des veines rétiniennes, associé à une imprégnation par la fluorescéine de la paroi des vaisseaux (staining) (issue de la bibliothèque d'images du service d'ophtalmologie de Rouen). 
En angiographie, on va pouvoir distinguer des : lésions artérielles, lésions veineuses, lésions inflammatoires de la paroi, lésions occlusives (occlusion de l'artère ou de la veine centrale de la rétine), nodules cotonneux (lésions blanches de petites tailles, traduisant une occlusion des artérioles pré-capillaires rétiniennes, également visibles au fond d'oeil) (Figure 8) (4).

Les vascularites rétiniennes veineuses peuvent s'observer au cours de la maladie de Behçet, de la sarcoïdose ou encore de la rétinochoroïdopathie de birdshot. En revanche, les vascularites artérielles sont plutôt l'apanage des causes infectieuses (virus de la famille herpes), de la maladie de Behçet et du lupus érythémateux systémique.

L'angiographie au vert d'indocyanine va venir compléter l'angiographie à la fluorescéine, en apportant des informations sur les structures chorö̈diennes (1). En effet, la molécule de vert d'indocyanine produit une fluorescence qui va pouvoir traverser l'épithélium pigmentaire rétinien, et ainsi, obtenir des images de la choroïde. Les zones d'hypofluorescences de grande taille sont évocatrices d'une non-perfusion choriocapillaire (atrophie choriorétinienne). Les taches hypofluorescentes de distribution régulière correspondent à des foyers de choroïdites. Les hyperfluorescences, quant à elles, sont en faveur d'une vascularite choroïdienne.

Lors de la rétinochoroïdopathie de birdshot, on observe de nombreuses taches hypofluorescentes en nasale et en inférieure de la papille, caractéristiques du diagnostic. Lors de la maladie de Vogt-Koyanagi-Harada, une vascularite choroïdienne sera visible précocement, associée à des taches hypofluorescentes de distribution régulière (1).

Le dernier paramètre étudié lors de l'examen ophtalmologique initial, signant la gravité de l'uvéite, est l'œdème maculaire. Il correspond à un phénomène d'exsudation de fluide intrarétinien (1). Il est qualifié de cystoïde lorsqu'il revêt un aspect kystique. Il peut être visualisé lors de l'examen au fond d'œil et à l'angiographie, mais son analyse repose essentiellement sur la tomographie en cohérence optique ou Optical coherence tomography (OCT). Le principe de l'OCT repose sur un phénomène optique, combinant l'analyse de longueurs d'onde de la lumière de référence et de la lumière réfléchie par les structures de l'œil. Les propriétés de réflexion et d'absorption de la lumière par les tissus de l'œil sont la source de contraste des images d'OCT, révélatrices d'informations morphologiques. L'OCT produit des images en coupe axiale de la rétine (1) (Figure 9). L'œdème maculaire est une complication fréquente de la maladie de birdshot, des uvéites idiopathiques ou de la maladie de Behçet (1). 


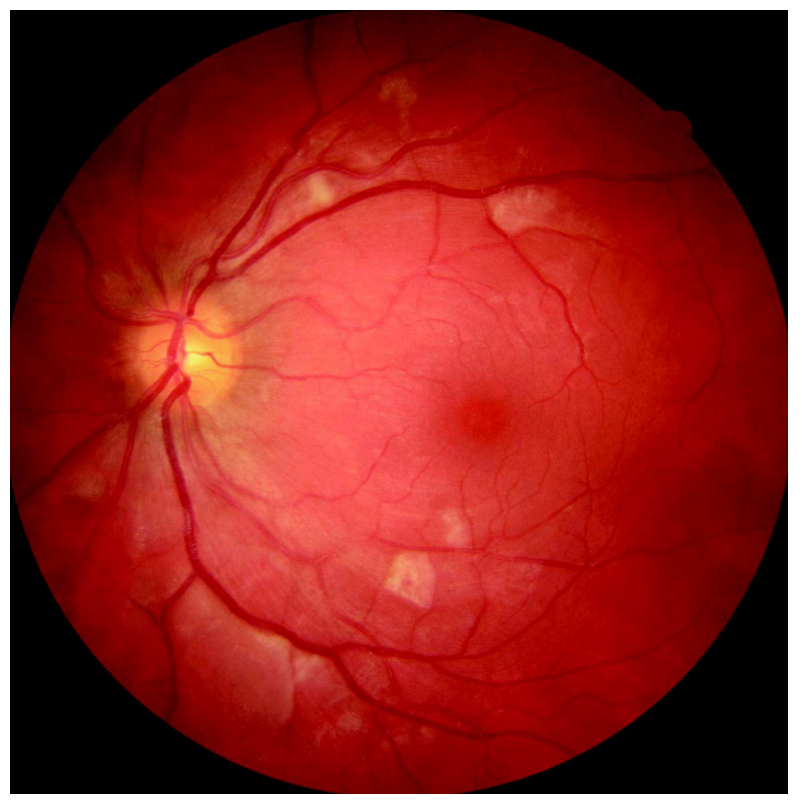

Figure 8 : Nodules cotonneux visualisés au fond d'œil (issue de la bibliothèque d'images du service d'ophtalmologie de Rouen).

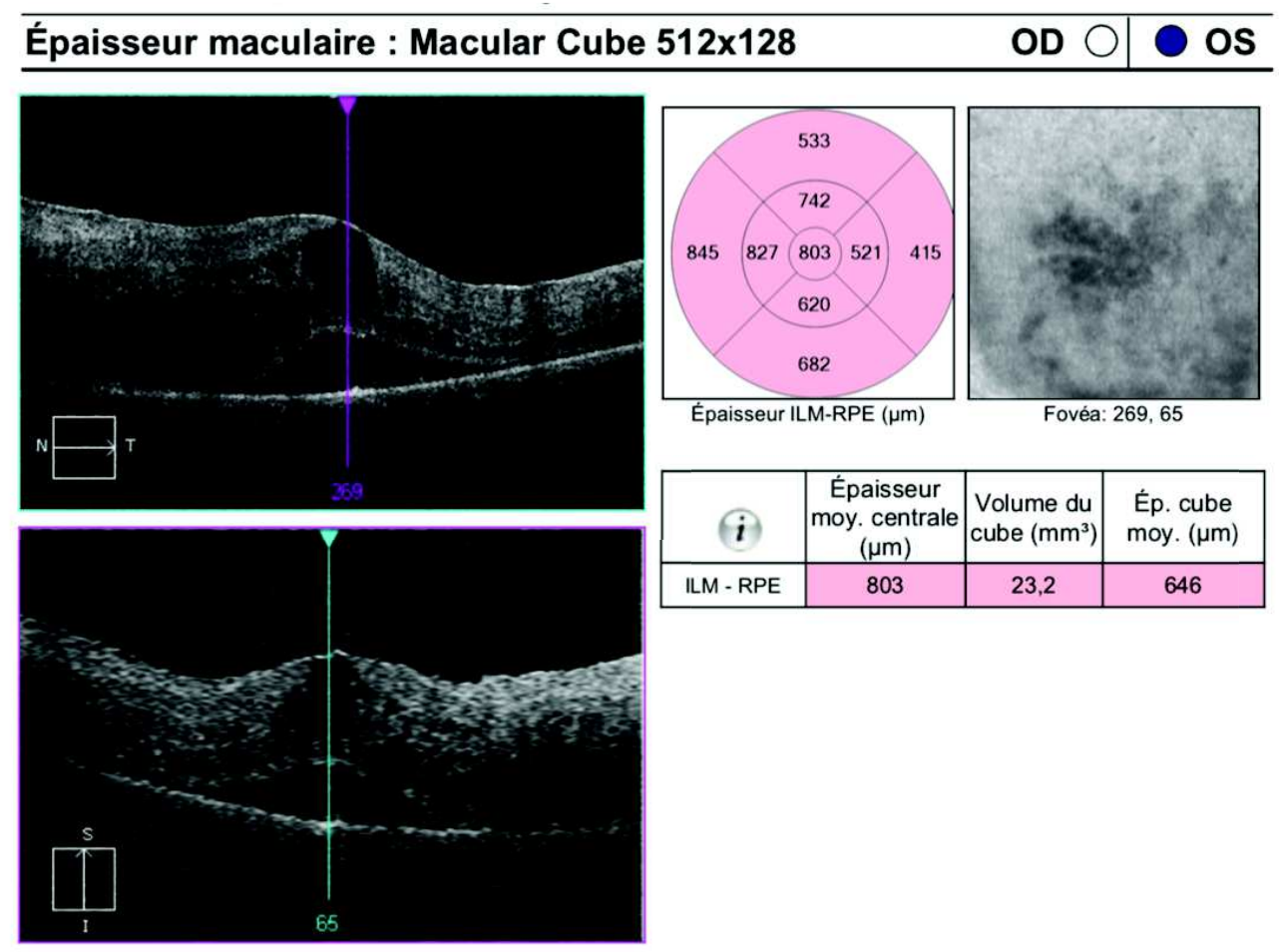

Figure 9 : Aspect d'œdème maculaire cystoïde à l'OCT : épaisseur maculaire centrale $>300 \mu \mathrm{m}$ et présence de logettes intra-rétiniennes (issue de la bibliothèque d'images du service d'ophtalmologie de Rouen). 


\subsection{Données épidémiologiques}

Les données épidémiologiques sont dépendantes de nombreux éléments : difficulté de nomenclature, variabilité géographique et hétérogénéité des populations comparées. La plus grande étude épidémiologique permettant d'estimer la prévalence des uvéites est américaine, réalisée sur une période de 1 an, à partir des données issues d'une base administrative (12). Parmi les 4 millions d'individus étudiés, 5299 patients présentaient une uvéite, majoritairement d'origine non-infectieuse pour $91 \%$ d'entre eux, soit 4827 patients. La prévalence des uvéites non-infectieuses était de 121 cas pour 100000 personnes. Quatre-vingt-un pour cent des uvéites non-infectieuses étaient de localisation antérieure. De ce fait, 923 patients présentaient une uvéite non-infectieuse non-antérieure, soit une prévalence de 23 pour 100000 habitants. Parmi ces patients, on dénombrait 44 uvéites intermédiaires, soit une prévalence de 1 pour 100000 habitants, 416 uvéites postérieures (10 pour 100000 habitants) et 463 panuvéites (12 pour 100000 habitants) (12). Dans les pays développés, les uvéites seraient à l'origine de 5 à $20 \%$ des cas de cécité légale, définie aux États-Unis comme une acuité visuelle du meilleur œil $\leq 1 / 10$ et selon l'Organisation mondiale de la santé (OMS) une acuité visuelle du meilleur œil $\leq 1 / 20$.

Les uvéites peuvent atteindre toutes les catégories d'âge. Les uvéites surviennent entre 20 et 50 ans dans 60 à $80 \%$ des cas (7). En revanche, on note certaines spécificités diagnostiques liées à l'âge. Les uvéites pédiatriques sont majoritairement secondaires aux arthrites juvéniles idiopathiques, alors que les uvéites des sujets âgés de plus de 50 ans correspondent, en grande partie, à la rétinochoroïdopathie de birdshot.

Les principales données épidémiologiques concernant les uvéites non-infectieuses sont issues de deux études. L'étude de Barisani-Asenbauer et al. (9), parue en 2012, rapporte les résultats d'une cohorte longitudinale, incluant de façon rétrospective l'ensemble des cas d'uvéites de 1995 à 2009 d'un centre tertiaire. Parmi les 2619 patients, 834 patients (31,8\%) présentaient une uvéite associée à une maladie de système extra-oculaire (465 patients) ou à une maladie intraoculaire stricte mais présumée de mécanisme immunologique (369 patients). Les étiologies les plus fréquemment retrouvées étaient les spondylarthropathies et les uvéites associées à l'haplotype HLA B27, sans manifestation rhumatologique. Concernant les maladies systémiques, les deux principales étiologies étaient représentées par la sarcoïdose (64 cas) et la maladie de Behçet (49 cas).

Bodaghi et al. ont inclus, de manière rétrospective, des patients présentant une uvéite chronique sévère. Elles étaient définies comme évoluant depuis plus de 3 mois, avec une acuité 
visuelle maximale $<2 / 10$ et ayant nécessité, au cours de leurs évolutions, le recours à un traitement immunosuppresseur systémique (corticoïdes ou autre) (10). Le nombre total de patients étaient de 927 : 28,5\% de cas d'uvéites antérieures, $15 \%$ d'uvéites intermédiaires, 21,5\% d'uvéites postérieures et $35 \%$ de panuvéites. La répartition étiologique était divisée en trois tiers : 34,2\% d'uvéites d'origine immunologique (soit dans le cadre d'une maladie systémique, soit celui d'une maladie immunologique cantonnée à l'œil), 31,8\% d'uvéites infectieuses et $34 \%$ d'uvéites idiopathiques. Trente pour cent des panuvéites étaient secondaires à une maladie de système, contre $14 \%$ des uvéites intermédiaires ou encore $6,5 \%$ des uvéites postérieures. Dans cette étude, l'évolution des uvéites sévères chroniques était favorable dans $59 \%$ des cas, avec une stabilisation de la situation ophtalmologique dans $22 \%$ des cas et enfin une dégradation de l'acuité visuelle dans $19 \%(10)$.

La baisse de l'acuité visuelle observée au cours des uvéites est principalement due au développement de complications oculaires, tels l'apparition d'une cataracte, d'un glaucome ou encore d'un œdème maculaire. Dans une cohorte de patients atteints d'uvéites non-infectieuses non-antérieures, Dick et al. (13) ont montré, sur une période de recul de 14 ans, l'apparition d'une cataracte chez $25 \%$ des patients, d'un glaucome chez $15 \%$ et d'une atteinte rétinienne (incluant le développement d'un œdème maculaire cystoïde, de néo-vaisseaux rétiniens, d'ischémie rétinienne) chez $23 \%$ des patients. Dans cette même étude, les auteurs ont montré que ces patients, atteints d'uvéites non-infectieuses non-antérieures, étaient plus à risque de développer des complications oculaires que des témoins (sujets de même âge et de même sexe, sans pathologies oculaires chroniques) : $58 \%$ des patients développaient une complication oculaire (cataracte, glaucome ou pathologie rétinienne) versus $17 \%$ des témoins, soit un risque relatif évalué à 5,2 (13).

Enfin, lors d'une étude prospective incluant 255 patients (14), les auteurs ont montré que les facteurs associés à une aggravation de l'acuité visuelle au cours des uvéites inflammatoires étaient : la durée d'évolution de la maladie à l'inclusion supérieure à 6 ans, la présence d'une cataracte dès l'inclusion, l'aggravation de l'inflammation en chambre antérieure (passage du grade 0 au grade 1+), l'aggravation ou la persistance de l'inflammation intravitréenne. En revanche, la localisation de l'uvéite n'apparaissait pas comme un facteur de risque.

Les uvéites non-infectieuses apparaissent comme une cause majeure de cécité dans les pays industrialisés, touchant dans la moitié des cas des adultes jeunes, responsable d'une morbidité significative. Par conséquent, la gestion de ces uvéites et de leurs complications se 
révèle, aujourd'hui, comme un nouvel enjeu. Leur traitement doit non seulement s'articuler autour du contrôle de l'inflammation intraoculaire et du nombre de poussées, mais également autour des comorbidités extra-oculaires, d'origine, pour partie, iatrogène.

\subsection{Les uvéites non-infectieuses : entité clinique aux nombreux diagnostics étiologiques}

Le cadre étiologique des uvéites inflammatoires est vaste. Cette étendue nécessite une analyse anamnestique et sémiologique fine, assurée par la collaboration étroite des ophtalmologistes et des internistes. Une étude de 2017, randomisée, multicentrique, ouverte, de non infériorité, avait pour but d'étudier la stratégie diagnostique des uvéites (15). Un groupe de patients bénéficiait d'un bilan étiologique dit « libre » où l'ophtalmologiste avait la possibilité de réaliser les explorations complémentaires de son souhait et d'adresser ou non, le patient à un interniste. Le deuxième groupe bénéficiait d'un bilan paraclinique «standardisé », consistant en une prise en charge conjointe par un ophtalmologiste et un interniste et la réalisation d'examens complémentaires hiérarchisés selon la symptomatologie ophtalmologique et extraophtalmologique. Six cent soixante-seize patients ont été inclus : 373 dans le bras « libre » et 303 dans le bras « standardisé ». Un diagnostic étiologique a été posé chez 203 patients $(54,4 \%)$ ayant bénéficié d'une démarche diagnostique libre et chez 150 patients $(49,5 \%)$ ayant suivi une stratégie standardisée $(p=0,20)$. En revanche, deux fois moins d'examens complémentaires étaient réalisés dans le groupe standardisé (2609 versus 5371, soit $\mathrm{p}<0,0001)$.

Ainsi, le bilan étiologique des atteintes inflammatoires oculaires doit être orienté par la sémiologie oculaire, mais doit également reposer sur une prise en charge globale axée sur une connaissance des manifestations cliniques des maladies systémiques les plus fréquemment rencontrées. L'impact d'une prise en charge collaborative permettant des explorations complémentaires ciblées s'intègre dans les perspectives hospitalières actuelles.

Nous décrirons ici uniquement les étiologies abordées dans notre travail, c'est-à-dire les étiologies rencontrées au cours des uvéites intermédiaires, postérieures et panuvéites noninfectieuses de l'adulte. Il s'agit donc d'une description non exhaustive, avec notamment l'absence de description de certaines pathologies fréquentes, telles les arthrites juvéniles idiopathiques (population pédiatrique) ou encore les uvéites entrant dans le cadre des spondylarthropathies (atteinte du segment antérieur). 


\subsubsection{La maladie de Behçet}

La maladie de Behçet est une vascularite systémique à la frontière entre les maladies auto-immunes et les syndromes auto-inflammatoires. Les manifestations oculaires sont très fréquentes au cours de la maladie de Behçet, décrites dans 28 à $70 \%$ des cas (16). L'atteinte oculaire fait d'ailleurs partie des critères diagnostiques de la maladie de Behçet, que ce soit au niveau des critères ISG (International study group) de 1990 ou les critères ICBD (International criteria for Behçet's disease) de 2013 (1).

L'atteinte la plus fréquemment retrouvée est une panuvéite dans 76 à $89 \%$ des cas (17). L'uvéite est systématiquement de type non granulomateuse. L'atteinte oculaire est volontiers sévère, avec une acuité visuelle moyenne de 0,75 LogMAR, soit 2/10 (18). Cette mise en jeu du pronostic visuel est secondaire aux atteintes du segment postérieur à type de vascularites rétiniennes, observées dans plus de $80 \%$ des cas. Les vascularites au cours de la maladie de Behçet peuvent être artérielles ou veineuses. Dans une revue récente, Bazvand et al. (18) ont montré que $86 \%$ des 51 patients de l'étude présentaient des lésions de vascularites. Neuf pour cent des atteintes correspondaient à des lésions veineuses, $6 \%$ à des atteintes artérielles, et pour la majorité des patients (71\%) l'atteinte était mixte. A l'inverse, les atteintes isolées du segment antérieur de l'œil sont rares, de l'ordre de 5 à $7 \%$. Un hypopion peut être retrouvé et il s'agit fréquemment d'une uvéite synéchiante.

La fréquence et la sévérité de l'atteinte oculaire au cours de la maladie de Behçet a amené la communauté scientifique à proposer des recommandations thérapeutiques. Les recommandations de l'EULAR (European league against rheumatism) publiées en avril 2018 (19), précisent que toute atteinte du segment postérieur au cours de la maladie de Behçet nécessite un traitement immunosuppresseur de type azathioprine, cyclosporine A, interféron alpha-2a ou anti-TNF alpha (Tumor necrosis factor alpha), pouvant être associé à une corticothérapie systémique. Pour les patients présentant une forme sévère menaçant le pronostic visuel, un traitement par fortes doses de corticoïdes, pouvant être d'emblée associé à de l'interféron alpha-2a ou de l'infliximab (anti-TNF alpha) est recommandé.

\subsubsection{La rétinochoroïdopathie de birdshot}

La maladie de birdshot correspond à une uvéite idiopathique bilatérale postérieure chronique, se compliquant dans $50 \%$ des cas d'un œdème maculaire. Les spécialistes en uvéites inflammatoires estiment que la maladie de birdshot représentent 0,5 à 1,5\% de leurs consultations, soit une prévalence estimée de 0,2 à 1,7 cas/100 000 personnes (20). Le 
diagnostic repose sur les critères suivants : le caractère bilatéral, la présence d'au moins trois lésions « de type birdshot» (taches au fond d'œil évocatrices d'une explosion de grenade au plomb) localisées en inférieur ou en nasal de la papille, le faible degré d'inflammation antérieure et intermédiaire, la fréquence de l'association à l'haplotype HLA A29, la présence d'une vascularite rétinienne et l'absence de synéchies postérieures (critère d'exclusion) (20). Contrairement à l'uvéite de la maladie de Behçet où l'acuité visuelle est souvent très altérée, la baisse d'acuité visuelle n'est pas constante au cours de la maladie de birdshot. Les symptômes ophtalmologiques sont plus divers, à type de troubles de la vision des couleurs, myodésopsies ou encore altération du champ visuel. L'angiographie à la fluorescéine retrouve une vascularite rétinienne avec une atteinte veineuse et capillaire, et l'angiographie au vert d'indocyanine confirme les taches hypofluorescentes en nasal et en inférieur, visualisées au fond d'œil.

La prise en charge thérapeutique de la maladie de birdshot n'est pas codifiée. Le traitement de première intention reste la corticothérapie, mais les études de cohorte de longue durée ont mis en évidence un contrôle insuffisant fréquent sous corticoïdes seuls (21). Si la ciclosporine était l'immunosuppresseur de première intention initialement, le mycophénolate mofétil s'est imposé au cours de ces dernières années (22). Suite aux travaux de Tomkins-Netzer et $a l$. la tendance actuelle est d'introduire d'emblée un traitement immunosuppresseur pour une durée prolongée. En effet, cette équipe a montré qu'un traitement prolongé par immunosuppresseur conventionnel (méthotrexate, mycophénolate mofétil, azathioprine ou cyclosporine), comparé à une corticothérapie courte (inférieure à 1 an), améliorait l'acuité visuelle lors de l'évaluation à deux ans du diagnostic (23).

\subsubsection{La choroïdite multifocale idiopathique}

Il s'agit d'une affection chronique choroïdienne inflammatoire rare, touchant majoritairement les femmes jeunes atteintes de myopie (1). Sur le plan ophtalmologique, il est fréquemment retrouvé une atteinte inflammatoire du segment antérieur, une atteinte variable du vitré, mais surtout une évolution vers une fibrose sous-rétinienne et une néovascularisation choroïdienne (24). L'acuité visuelle initiale est souvent préservée. Le diagnostic se fait lors du fond d'œil avec la mise en évidence de taches jaunes au niveau de l'épithélium pigmentaire, de taille variable (de 50 à $1000 \mu \mathrm{m}$ ), localisées en périphérie, ainsi qu'au pôle postérieur. L'angiographie à la fluorescéine retrouve une hypofluorescence précoce, puis une imprégnation tardive des taches choroïdiennes. Au vert d'indocyanine, ces taches sont hypofluorescentes dès les temps précoces et cette hypofluorescence va persister au cours de l'examen. Une atteinte du 
champ visuel, à type d'élargissement de la tache aveugle ou de déficits périphériques temporaux, peut être rencontrée (1).

La stratégie thérapeutique va être modulée par la présence de néo-vaisseaux. En l'absence de néo-vaisseaux, un traitement par corticothérapie seule sera privilégié. L'ajout d'un traitement immunosuppresseur, en premier lieu le mycophénolate mofétil, a montré une diminution des infiltrats inflammatoires rétiniens (24). En cas d'apparition de néo-vaisseaux, les anti-angiogéniques (anti-VEGF, Vascular endothelial growth factor) sont alors indiqués. Le pronostic visuel de cette entité est réservé.

\subsubsection{La sarcoïdose}

La sarcoïdose est une maladie systémique de physiopathologie partiellement élucidée, mais en partie médiée par une infiltration lymphocytaire $\mathrm{T}$, responsable de la formation de granulomes épithéloïdes et giganto-cellulaires (25). La sarcoïdose affecte les patients de tout âge et de toutes origines ethniques. On relève une incidence plus importante chez les patients afro-américains (35 nouveaux cas/100 000 personnes par an) que chez les caucasiens (5-10 nouveaux cas/100 000 personnes par an) (26). L'atteinte ophtalmologique est présente dans 25 à $50 \%$ des cas (27). Les uvéites constituent la manifestation la plus fréquente, suivies des kératites sèches dues à l'atteinte des glandes lacrymales et les atteintes orbitaires. Les uvéites sarcoïdosiques représentent 2 à $15 \%$ des uvéites nouvellement diagnostiquées (28).

Les uvéites sont le plus souvent de localisation antérieure, et sont fréquemment de type granulomateuse (1). Les uvéites intermédiaires sont retrouvées dans 10 à $20 \%$ des cas, avec une hyalite caractéristique en œuf de fourmi. Les uvéites postérieures sont notées dans 10 à $40 \%$ des cas, caractérisées par une vascularite le plus souvent veineuse. Enfin, on relève une panuvéite dans 9 à $30 \%$ des cas. Toute la difficulté dans le diagnostic de la sarcoïdose repose sur le caractère non spécifique des atteintes et l'absence de preuve histologique systématique. Un consensus international IWOS (International workshop on ocular sarcoidosis) (29) a récemment revu les critères diagnostiques des atteintes oculaires de la sarcoïdose. Les auteurs ont ainsi classé le diagnostic en prouvé, présumé et probable, en fonction des signes cliniques, biologiques et histologiques.

Le traitement de l'uvéite sarcoïdosique va dépendre d'une part, de la localisation de l'uvéite, et d'autre part, des manifestations extra-oculaires associées. Pour les uvéites postérieures et panuvéites, le traitement de première intention s'appuie sur une corticothérapie orale. Devant un échec de la corticothérapie ou une cortico-dépendance, un traitement de 
deuxième ligne sera indiqué dans 1 à $23 \%$ des cas (30). Le traitement alors utilisé est le méthotrexate, à partir des données de l'étude de Baughman (31). Une efficacité thérapeutique était observée chez $90 \%$ des patients (dont $13 \%$ ont pu arrêter le traitement), $7 \%$ des patients ont présenté un échec du méthotrexate et enfin, $3 \%$ ont dû arrêter le traitement pour effets indésirables. Le mycophénolate mofétil trouve également sa place dans cette indication, mais les données actuelles reposent sur des études de petit effectif (32). Une efficacité des anti-TNF alpha a été montrée chez les patients ayant présenté un échec à au moins un immunosuppresseur conventionnel (33). Le pronostic visuel reste sévère puisque, pour $10 \%$ des patients, on observera au cours du suivi une altération de l'acuité visuelle $\leq 1 / 10$ pour un œil (28).

\subsubsection{La maladie de Vogt-Koyanagi-Harada}

La maladie de Vogt-Koyanagi-Harada est une panuvéite granulomateuse bilatérale, associée à des décollements séreux rétiniens, d'origine auto-immune dont la cible antigénique est le mélanocyte (1). Contrairement à la maladie de birdshot, les manifestations de la maladie ne sont pas uniquement ophtalmologiques, et vont être associés, des signes neurologiques (méningite lymphocytaire initiale et transitoire), une surdité de perception réversible et des signes cutanées (vitiligo et poliose, plus tardifs) (34). Il s'agit d'une atteinte auto-immune médiée par les lymphocytes T de type T helper (Th) 17, responsables de la production d'IL-17. Ces lymphocytes $\mathrm{T}$ auto-réactifs vont reconnaître les tyrosinase-related proteins des mélanocytes (34).

Cliniquement, les patients se présentent avec une baisse d'acuité visuelle sévère, souvent bilatérale. L'examen ophtalmologique retrouve une panuvéite, associée à un œdème papillaire, avec des décollements séreux rétiniens, débutant au niveau de la macula et pouvant s'étendre en périphérie, confirmés par l'OCT (1). Des lésions choroïdiennes peuvent également être retrouvées, confirmées en angiographie. A l'angiographie au vert d'indocyanine, les signes précoces de la maladie vont montrer des signes de vascularite choroïdienne (fuite à partir des vaisseaux choroïdiens aux temps précoces et vaisseaux choroïdiens hyperfluorescents aux temps tardifs), associés à des taches hypofluorescentes de distribution régulière (1).

Le traitement de première intention repose sur une corticothérapie générale. Certains auteurs soulignent l'intérêt de débuter d'emblée un traitement immunosuppresseur, notamment le mycophénolate mofétil, qui assurerait un effet bénéfique sur le maintien de l'acuité visuelle et le risque de chronicité (35). La place de l'azathioprine dans le contrôle de l'inflammation oculaire, tant à la phase initiale que finale, a également été soulignée (36). Quelques auteurs 
ont rapporté l'efficacité des anti-TNF alpha en cas d'échec des traitements immunosuppresseurs conventionnels (37), mais la maladie de Vogt-Koyanagi-Harada présente une réponse globalement satisfaisante à la corticothérapie ou aux immunosuppresseurs conventionnels.

\subsection{Les uvéites non-infectieuses : de la physiopathologie à la prise en charge thérapeutique}

L'avancée sur la compréhension des mécanismes physiopathologiques dans les uvéites a été permise par les modèles animaux expérimentaux d'uvéite auto-immune expérimentale (EAU), principalement chez la souris et chez le rat. Le modèle de l'EAU repose sur une immunisation active par les peptides de la rétine ou une immunisation passive par transfert de lymphocytes $\mathrm{T}$ auto-réactifs. Actuellement, l'immunisation active repose sur l'injection de différentes variétés de fragments peptidiques immunogènes tels l'IRBP (Interphotoreceptor retinoid binding protein) ou l'Ag-S (Antigène soluble rétinien), de l'adjuvant complet de Freund (assurant une libération lente de l'antigène à partir de l'inoculum) et de toxine pertussique (agent immun adjuvant). Les souris vont développer une maladie monophasique, touchant essentiellement le segment postérieur de l'œil. A l'inverse, le rat va développer à la fois une maladie monophasique ou évoluant par poussées, et présentera plutôt une atteinte de type panuvéite (38).

Le bulbe oculaire apparaît comme un organe présentant un privilège immunologique. Ce privilège est en parti assuré par une barrière hémato-rétinienne efficace, et un système de drainage lymphatique limité. La barrière hémato-rétinienne est formée par l'endothélium vasculaire rétinien et les cellules de l'épithélium pigmenté de la rétine, de l'iris et du corps ciliaire (1). De plus, l'humeur aqueuse apparaît comme réfractaire au développement d'une réaction inflammatoire, devant sa richesse en molécules inhibitrices tels le TGF beta (Tumor growth factor beta), l'acide rétinoïde ou encore l'expression accrue de Fas Ligand par les cellules rétiniennes (39). Comment les cellules du système immunitaire vont-elles envahir cet environnement ? La principale hypothèse actuelle repose sur l'activation périphérique de lymphocytes $\mathrm{T} \mathrm{CD}^{+}$auto-réactifs, spécifiques de peptides rétiniens, ayant échappé aux procédés de tolérance centrale. Cette activation pourrait faire intervenir un mécanisme de mimétisme moléculaire entre un antigène viral non identifié et un antigène rétinien. Ces lymphocytes $\mathrm{T}$ activés vont alors traverser la barrière hémato-rétinienne et adhérer à l'endothélium vasculaire, modifiant la structure des veinules rétiniennes. En effet, seuls les 
lymphocytes $\mathrm{T}$ activés ont la capacité de traverser cette barrière. Puis, ils vont sécréter des cytokines responsables du recrutement des autres effecteurs du système immunitaire (lymphocytes $\mathrm{T} \mathrm{CD} 8^{+}$, macrophages, polynucléaires neutrophiles) et vont également amplifier la destruction de la barrière hémato-rétinienne par la sécrétion de métallo-protéases. L'activation de ces lymphocytes $\mathrm{T} \mathrm{CD}^{+}$auto-réactifs sera maintenue grâce à une nouvelle stimulation par les auto-antigènes rétiniens. Cette stimulation sera assurée par les cellules dendritiques résidentes de l'œil (39) (Figure 10).

Les uvéites apparaissent comme des maladies dépendantes des lymphocytes T CD4 ${ }^{+}$. Le mécanisme physiopathologique du développement des uvéites a été initialement attribué à une réponse immunitaire Th1. Cependant, les modèles animaux ont ensuite remis en question cette polarisation. En effet, les souris déficientes en interféron gamma vont développer les signes cliniques de l'EAU. Une nouvelle population de lymphocytes $\mathrm{T}$ est apparue comme impliquée dans la physiopathologie des uvéites. Il s'agit des lymphocytes Th17 producteurs d'IL-17. Le transfert de lymphocytes Th1 et Th17 induit la maladie chez des souris sauvages. Dans les modèles animaux, l'interaction entre les lymphocytes Th1 et Th17 est complexe, influencée par l'environnement cytokinique (40).

Les uvéites apparaissent comme un modèle de maladie immunologique impliquant les acteurs de l'immunité innée et adaptative. Les TLR (Toll-like receptor) semblent jouer un rôle important dans le déclenchement de la maladie. Les souris déficientes en TLR2, 4 et 9 sont plus susceptibles au développement de l'EAU induit par immunisation par l'IRBP. Au cours de l'EAU, il a été observé une augmentation de l'expression des récepteurs CCR2, CCR5 et CX3CR1 impliqués dans le recrutement des macrophages en favorisant leur capacité à traverser la barrière hémato-rétinienne (38).

Sur le plan cytokinique, le TNF alpha (Tumor necrosis factor) apparaît comme une cytokine majeure dans la physiopathologie des uvéites. Le TNF alpha orchestre l'infiltration oculaire par les lymphocytes T et les macrophages. De plus, le TNF alpha assure la maturation et la survie des macrophages et engendre la production de monoxyde d'azote, responsable des lésions rétiniennes. Le blocage du TNF alpha et de son récepteur a montré son efficacité dans les modèles murins d'EAU. Les souris knock-out KO pour le récepteur 1 au TNF alpha présentent une augmentation de l'apoptose des macrophages et, de ce fait, une diminution du nombre de cellules immunitaires au site inflammatoire (38). 


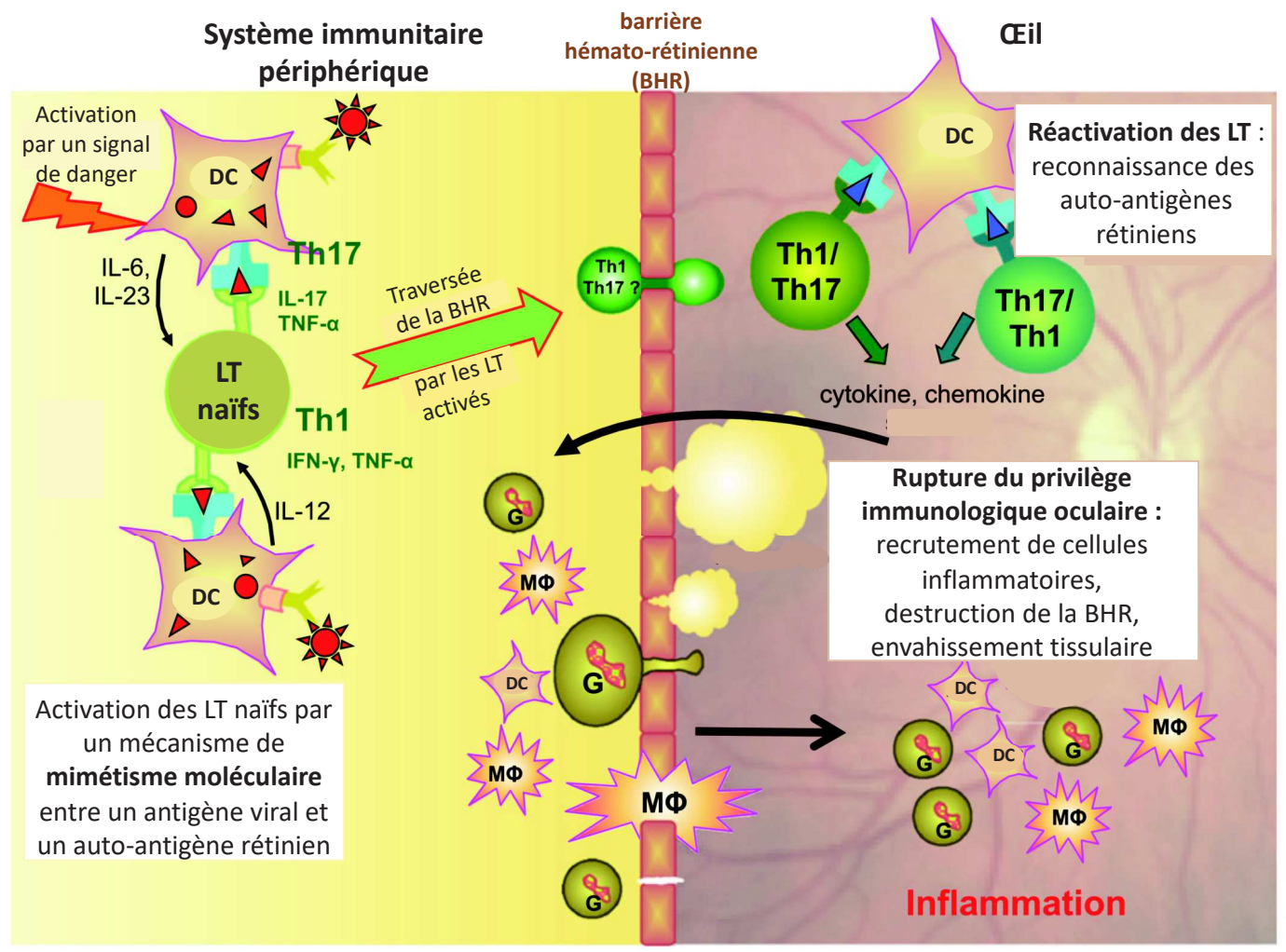

Figure 10 : Physiopathologie des uvéites non-infectieuses [d'après Bose et al. Autoimmun Rev, 2016 (38)]. DC : cellule dendritique. LT : lymphocyte T. G : granulocyte. MФ : macrophage. 
L'IL-6 est une cytokine également impliquée dans la physiopathologie des uvéites. Les modèles murins déficients en IL-6 vont développer une maladie moins sévère. L'IL-6 présente non seulement une action pro-inflammatoire, mais joue également un rôle dans les phénomènes d'exsudation vasculaire, en favorisant la sécrétion anormale de VEGF, responsable de la formation d'œdème maculaire (41). Enfin, l'IL-10 apparaît comme une cytokine régulatrice majeure, assurant notamment une polarisation des macrophages en phénotype régulateur M2. Dans les modèles murins d'EAU, un défaut de production de cytokines régulatrices, dont l'IL- 10, a été mis en évidence (41).

Chez l'homme, des taux élevés de TNF alpha, d'IL-17 et d'IL-6 ont été relevés dans l'humeur aqueuse de patients atteints de maladie de birdshot, de maladie de Vogt-KoyanagiHarada et de maladie de Behçet.

Enfin, un défaut de tolérance est actuellement souligné dans la pathogénicité des uvéites. Un défaut de tolérance centrale tout d'abord, devant un défaut de sélection négative des lymphocytes auto-réactifs vis-à-vis des antigènes rétiniens. Un défaut de tolérance périphérique est également impliqué. En effet, les souris déficientes en lymphocytes T régulateurs vont développer une maladie plus sévère. Dans l'EAU, les lymphocytes T régulateurs apparaissent comme ayant une fonctionnalité diminuée et comme étant dans l'incapacité de contrôler les lymphocytes auto-réactifs d'origine périphérique (42).

\subsection{Les uvéites non-infectieuses : des stratégies thérapeutiques en pleine évolution}

Les objectifs de la prise en charge thérapeutique des uvéites non-infectieuses sont multiples : contrôler rapidement et efficacement l'inflammation, afin de préserver la fonction visuelle et limiter les dommages structuraux irréversibles, prévenir le risque de rechute, être efficace sur les atteintes extra-ophtalmologiques en cas d'étiologie systémique et enfin, limiter les effets indésirables ophtalmologiques et généraux d'origine iatrogène (43).

Le traitement repose en première intention sur une corticothérapie per os à $1 \mathrm{mg} / \mathrm{kg}$, pouvant être précédée par des bolus intraveineux en fonction de la sévérité de l'uvéite. En cas d'intensification thérapeutique, un traitement par immunosuppresseur conventionnel ou biothérapie semble être préférable à un traitement par implant intra-vitréen de corticoïdes. En effet, l'étude randomisée MUST (Multicenter uveitis steroid treatment) a mis en évidence, avec un recul de 7 ans, une supériorité des traitements immunosuppresseurs conventionnels ou biothérapies versus un implant de fluocinolone, uni- ou bilatéral. Cette supériorité concernait 
l'amélioration de l'acuité visuelle. Dans le groupe de patients traités par implant, après 7 ans de suivi, il a été observé une augmentation de $8 \%$ du nombre de patients présentant une cécité (acuité visuelle $\leq 1 / 10$ ) par rapport à l'initiation du traitement. Dans le groupe traité par immunosuppresseur conventionnel, une diminution de $1 \%$ du nombre de patients aveugles était montrée (44). L'effet bénéfique du traitement immunosuppresseur portait également sur l'amélioration de l'œdème maculaire cystoïde initial et sur les effets secondaires oculaires (cataracte et glaucome).

La première interrogation est de déterminer les circonstances devant amener à intensifier le traitement, par immunosuppresseurs conventionnels ou biothérapies. Actuellement, un groupe de consensus international recommande d'introduire ces traitements pour contrôler une inflammation persistante ou sévère, dans l'objectif de limiter l'apparition de complications (45). Ces thérapeutiques pourront également être introduites à visée d'épargne cortisonique (uvéite nécessitant une forte dose de corticoïdes pour être inactive appelée cortico-dépendance) ou en cas de mauvaise tolérance de la corticothérapie. Sous le terme d'inflammation sévère, les auteurs incluaient la présence d'un risque pour le pronostic visuel, une atteinte bilatérale, un œdème maculaire, une vascularite ou encore une névrite optique. Ainsi, ces recommandations encouragent le prescripteur à introduire, dès le diagnostic de l'uvéite, un traitement immunomodulateur en cas de critères de sévérité (45).

1.6.1. Le choix actuel de première intention, en cas d'intensification thérapeutique : les immunosuppresseurs conventionnels

En cas d'indication à une intensification thérapeutique, le choix actuel se porte sur les immunosuppresseurs conventionnels. Le groupe de consensus international a inclus dans ces traitements la ciclosporine, le tacrolimus, le méthotrexate, le mycophénolate mofétil et l'azathioprine. Le cyclophosphamide n'était pas inclus devant le faible niveau de preuve permettant de retenir son indication (45).

Actuellement, le seul traitement immunosuppresseur conventionnel ayant obtenu l'Autorisation de mise sur le marché (AMM) dans la prise en charge des uvéites noninfectieuses est la ciclosporine. La ciclosporine, tout comme le tacrolimus, appartient à la famille des inhibiteurs de la calcineurine. Ils vont agir spécifiquement au niveau des lymphocytes T activés, en inhibant la libération de la calcineurine induite par l'activation du 
TCR (T-cell receptor) et ainsi, en inhibant la translocation du facteur de transcription NFAT (Nuclear factor of activated T-cells) (46). Au niveau oculaire, ils ont montré leur efficacité en termes d'amélioration de l'acuité visuelle et de contrôle de l'inflammation (45). Si leur utilisation lors des transplantations d'organes est toujours d'actualité, elle est devenue beaucoup plus rare au cours des pathologies inflammatoires oculaires, devant leurs effets indésirables (néphrotoxicité...) (1).

Les principales données de la littérature portant sur l'efficacité du méthotrexate, du mycophénolate mofétil et de l'azathioprine au cours des atteintes inflammatoires oculaires, sont issues de la cohorte SITE (Systemic immunosuppressive therapy for eye diseases), étude rétrospective américaine incluant 8562 patients (47). Le méthotrexate est un analogue de l'acide folique qui enraye la synthèse de l'ADN en inhibant la synthèse des bases puriques et pyrimidiques. Il va également avoir une action limitant le chimiotactisme des polynucléaires neutrophiles (1). Le méthotrexate a montré son efficacité en matière de contrôle de l'inflammation, d'amélioration de l'acuité visuelle et d'épargne cortisonique (45). Dans la cohorte SITE, pour l'ensemble des pathologies inflammatoires oculaires incluses (uvéites mais aussi sclérites et autres atteintes inflammatoires oculaires), soit pour 384 patients, après un an de traitement, on relevait une efficacité du méthotrexate dans $66 \%$ des cas, $13 \%$ des patients avaient arrêté le traitement pour inefficacité et $16 \%$ pour effets indésirables (48).

Le mycophénolate mofétil est un inhibiteur de l'inosine-5-monophosphate déshydrogénase, enzyme clef de la voie de synthèse de novo des bases puriques, voie privilégiée au niveau des lymphocytes $\mathrm{T}$ et $\mathrm{B}$ activés. Il s'agit ainsi d'un puissant inhibiteur de la prolifération lymphocytaire (46). Tout comme le méthotrexate, le mycophénolate mofétil présente une action notable tant au niveau du contrôle de l'inflammation oculaire, de l'amélioration de l'acuité visuelle ou comme traitement d'épargne cortisonique (45). Parmi les 236 patients inclus dans la cohorte SITE, toute étiologie confondue, le mycophénolate mofétil a montré une efficacité dans $73 \%$ des cas, $13 \%$ des patients ont arrêté le traitement pour inefficacité après un an et $12 \%$ pour effets indésirables (49).

L'azathioprine est un analogue des bases puriques et agit comme inhibiteur de la synthèse de l'ADN. Son incorporation, à la place de l'adénine ou de la guanine, va être responsable d'une apoptose cellulaire. Il agit lui aussi comme inhibiteur de la prolifération lymphocytaire (46). A l'inverse du méthotrexate et du mycophénolate mofétil, si son action sur 
la résolution de l'inflammation oculaire et son rôle dans l'épargne cortisonique ont été démontrés, les données portant sur l'amélioration de l'acuité visuelle sous azathioprine sont manquantes (45). Les résultats de la cohorte SITE nous informent que, pour les 209 patients ayant reçu de l'azathioprine pendant au moins un an pour une atteinte inflammatoire orbitaire ou oculaire, une efficacité était retrouvée dans $73 \%$ des cas. A la fin du suivi (230 jours), un arrêt pour inefficacité était observé pour $17 \%$ des patients, et un arrêt pour effet indésirable dans $24 \%$ des cas (50).

L'étude de cohorte rétrospective de Galor et al. portant sur 257 patients et comparant l'efficacité du méthotrexate, du mycophénolate mofétil et de l'azathioprine, a mis en exergue une supériorité du mycophénolate mofétil avec un taux de succès après 6 mois de traitement respectivement de $42 \%, 70 \%$ et $58 \%$ pour ces 3 traitements (51). En pratique clinique, ces 3 traitements sont utilisés de manière homogène dans la prise en charge des uvéites inflammatoires non-infectieuses.

\subsubsection{Les thérapeutiques de deuxième recours : les biothérapies}

Parmi les biothérapies, les anti-TNF alpha constituent la classe thérapeutique la plus étudiée dans la prise en charge des uvéites inflammatoires. L'adalimumab, anticorps monoclonal humain bloquant l'interaction entre le TNF alpha et ses récepteurs, a d'ailleurs obtenu l'AMM en 2017 pour la prise en charge des uvéites intermédiaires, postérieures et panuvéites non-infectieuses présentant des signes d'activité persistants malgré une corticothérapie, ou pour les uvéites inactives cortico-dépendantes. Cette AMM repose sur deux études : les études VISUAL I et II. L'étude VISUAL I était une étude randomisée, en double aveugle, avec deux bras de traitement : un bras traité par adalimumab et un bras traité par placebo (52). A noter que dans les deux groupes, les patients pouvaient poursuivre un traitement immunosuppresseur (en dehors de l'interféron alpha-2a). La corticothérapie devait être sevrée au bout de 15 semaines. Deux cent dix-sept patients ont été inclus. Le critère de jugement principal était le délai de rechute. Ce délai était significativement plus court dans le groupe placebo (13 semaines) que dans le groupe adalimumab (24 semaines, $\mathrm{p}<0,001$ ), avec une diminution de moitié du risque d'échec thérapeutique. Les patients traités par adalimumab avaient un risque de développer une nouvelle lésion inflammatoire, de majoration de l'inflammation antérieure ou vitréenne ou de dégradation de l'acuité visuelle, significativement diminué par rapport au groupe placebo. Concernant l'étude VISUAL II (53), étaient inclus les patients présentant une uvéite inactive mais nécessitant de fortes doses de corticoïdes (entre 10 
et $35 \mathrm{mg} /$ jour) pour être contrôlée. La corticothérapie devait être sevrée au bout de 19 semaines. Le critère de jugement principal était également le délai de rechute. Deux cent vingt-six patients ont été inclus dans cette étude. Une rechute est survenue chez $55 \%$ des patients traités par placebo, avec un délai médian de rechute de 8,3 mois. Le risque de rechute était significativement diminué pour les patients traités par adalimumab $(p=0,004)$ puisqu'une rechute est survenue chez $39 \%$ des patients, avec un délai médian de rechute supérieur à 18 mois. Les patients traités par adalimumab avaient un risque de rechute secondaire à une dégradation de l'acuité visuelle significativement diminué par rapport au groupe placebo. La significativité n'était pas atteinte pour les autres paramètres de rechute étudiés (apparition d'une nouvelle lésion inflammatoire, majoration de l'atteinte inflammatoire antérieure ou vitréenne).

L'infliximab a également montré son efficacité dans la prise en charge des uvéites noninfectieuses, à travers des études prospectives ouvertes, notamment au cours de la maladie de Behçet. Il s'agit d'un anticorps monoclonal chimérique directement dirigé contre le TNF alpha. Dans une étude portant sur 10 patients atteints d'une maladie de Behçet active, les auteurs ont montré une efficacité précoce et rapide sur la réduction de l'inflammation intraoculaire et l'amélioration de l'acuité visuelle (54). Une deuxième étude, visant à évaluer l'efficacité à long terme de l'infliximab dans la maladie de Behçet, a montré que l'ensemble des patients $(n=21)$ présentaient une rémission clinique après un an de traitement (55). Ces données ont amené à recommander l'infliximab en première intention dans la prise en charge des patients atteints d'une maladie de Behçet avec atteinte oculaire menaçant le pronostic visuel (19).

Le tocilizumab est un anticorps monoclonal humanisé capable de se fixer à la fois sur le récepteur à l'IL-6 soluble ou membranaire. Ce traitement a notamment montré son efficacité dans la prise en charge des uvéites réfractaires (56). Un contrôle de l'uvéite avec une réduction de la posologie de corticoïdes $\leq 5 \mathrm{mg}$ /jour était obtenu dans $54 \%$ des cas après 6 mois de traitement et dans $58 \%$ des cas après 9 mois (56). Dans une étude ouverte, portant sur 37 patients atteints d'uvéites intermédiaires, postérieures ou panuvéites, les auteurs ont montré une efficacité du tocilizumab, tant sur le plan du contrôle de l'inflammation oculaire, de l'amélioration de l'acuité visuelle ou de la réduction de l'œdème maculaire, après 6 mois de traitement (57).

L'interféron alpha-2a (Roferon $\left.{ }^{\circledR}\right)$ a démontré son efficacité dans la prise en charge des uvéites non-infectieuses, particulièrement pour les uvéites compliquées d'œdème maculaire, ou 
encore au cours de la maladie de Behçet. Il s'agit d'un traitement possédant les mêmes propriétés que les cytokines naturelles, à savoir une activité anti-virale, anti-tumorale, anti-proliférative et immunomodulatrice (1). Dans une revue de la littérature reprenant 32 articles (58), une efficacité complète de l'interféron sur les manifestations oculaires du Behçet était retrouvée dans $75 \%$ des cas, et une efficacité partielle dans $19 \%$. Au cours d'une autre étude rétrospective portant sur 25 patients (59), les auteurs ont montré de façon significative une amélioration de l'acuité visuelle à un an de traitement, persistante à 2 ans, ainsi qu'une diminution du taux de rechute et une épargne cortisonique. Enfin, dans un essai thérapeutique français randomisé (60), incluant des uvéites postérieures compliquées d'œdème maculaire, les auteurs ont mis en évidence une efficacité de l'interféron sur la réduction de l'épaisseur maculaire centrale après 4 mois de traitement, mais cette différence n'était pas significative, comparée aux patients traités par corticothérapie seule.

\subsubsection{Problématiques thérapeutiques actuelles}

Ainsi, les biothérapies ont montré leur efficacité en termes de contrôle des uvéites et de prévention des rechutes. Cependant, certaines interrogations persistent (61). La première est de déterminer la place des biothérapies par rapport aux traitements immunosuppresseurs conventionnels, dans l'objectif double de préserver la fonction visuelle, tout en limitant l'exposition aux patients à des traitements dont le profil de tolérance reste encore à établir. La deuxième question est de déterminer les patients éligibles à ces nouvelles thérapeutiques, en fonction de leur diagnostic étiologique et de leurs comorbidités.

La troisième interrogation est celle du choix de la molécule, en fonction des connaissances physiopathologiques, mais également, en fonction du coût du traitement.

La quatrième question est celle de la durée du traitement, devant le risque de perte d'efficacité dans le temps, et également de déterminer la nécessité ou non, d'un schéma de décroissance. Les biothérapies doivent-elles rester un choix de deuxième ligne, derrière immunosuppresseurs conventionnels, ou doit-on les positionner plus en amont dans la stratégie thérapeutique ? 


\section{Matériels et méthodes}

Il s'agit d'une étude rétrospective monocentrique menée au CHU de Rouen. L'identification des patients a été effectuée en parallèle dans le service de médecine interne et d'ophtalmologie. En médecine interne, les dossiers ont été extraits à partir des codages CIM10 (Classification internationale des maladies, $10^{\text {ème }}$ version) (Annexe 1). En ophtalmologie, les dossiers ont été extraits à partir du logiciel de consultation Softalmo ${ }^{\circledR}$. L'ensemble des consultations entre avril 2014 (début informatisation) et septembre 2018 portant le codage « uvéite » ont été extraites.

\subsection{Critères d'inclusion}

Les critères d'inclusion de notre étude étaient les suivants :

- Patients majeurs au moment de l'inclusion

- Diagnostic d'uvéite d'origine non-infectieuse

- Uvéite de localisation intermédiaire, postérieure ou panuvéite

- Prise en charge thérapeutique de l'uvéite, au moment de l'inclusion, par un traitement immunosuppresseur conventionnel ou une biothérapie. Les traitements immunosuppresseurs conventionnels comprenaient le méthotrexate, le mycophénolate mofétil, l'azathioprine. Les biothérapies incluaient les anti-TNF alpha, dont l'infliximab et l'adalimumab, et l'anti-IL-6 récepteur, le tocilizumab

\subsection{Critères d'exclusion}

Les critères d'exclusion de notre étude étaient les suivants :

- Patients mineurs

- Présence d'une uvéite infectieuse

- Uvéite de localisation antérieure exclusive

- Prise en charge thérapeutique de l'uvéite, au moment de l'inclusion, par corticothérapie seule, quelle que soit sa forme

- Prise en charge thérapeutique de l'uvéite, au moment de l'inclusion, par de l'interféron alpha-2a 


\subsection{Définition des groupes et objectifs de l'étude}

L'objectif principal de l'étude était de comparer l'efficacité sur la prévention des récidives et la tolérance thérapeutique des biothérapies versus immunosuppresseurs conventionnels dans le cadre des uvéites non-infectieuses non-antérieures actives et/ou corticodépendantes. Pour cela, deux groupes ont été constitués : le groupe dit « immunosuppresseur conventionnel » et le groupe dit « biothérapie ». Étaient inclus dans le groupe immunosuppresseur conventionnel les patients traités au moment de l'inclusion par méthotrexate ou mycophénolate mofétil ou azathioprine. Les patients ne devaient jamais avoir bénéficié d'un traitement par anti-TNF alpha ou tocilizumab. Étaient inclus dans le groupe biothérapie, les patients traités au moment de l'inclusion par anti-TNF alpha ou tocilizumab, indépendamment de leur histoire thérapeutique antérieure. Les patients inclus dans ce groupe pouvaient avoir reçu, avant le traitement par biothérapie, un traitement par immunosuppresseur conventionnel ou interféron alpha-2a.

Le critère principal de jugement était un critère composite défini par l'apparition de l'événement rechute sous traitement ou l'arrêt du traitement pour un effet indésirable grave. Le taux de survie sans progression a été défini comme le taux de patients n'ayant pas présenté le critère de jugement principal.

L'évènement rechute était un événement ophtalmologique défini d'après les données de la littérature (52) : l'aggravation de l'acuité visuelle d'au moins trois lignes selon l'échelle ETDRS (Early treatment diabetic retinopathy study chart), par rapport au meilleur résultat antérieur (en dehors de l'apparition d'une cataracte) et/ou l'aggravation de l'inflammation intraoculaire, mesurée par les échelles du SUN (Tyndall protéique et flou vitréen), de deux niveaux par rapport au meilleur résultat antérieur et/ou l'absence de correction ou l'apparition d'une vascularite active et/ou l'absence de correction ou l'apparition d'un œdème maculaire cystoïde (défini comme une épaisseur maculaire centrale supérieure strictement à $300 \mu \mathrm{m}$ mesurée en OCT, associée à la visualisation de logettes intra-rétiniennes). La mesure de l'acuité visuelle était réalisée selon l'échelle de Monoyer. Pour l'analyse des résultats, l'acuité visuelle mesurée était ensuite exprimée selon l'échelle ETDRS et l'échelle LogMAR. Les différentes échelles d'évaluation de l'acuité visuelle sont précisées en Annexe 2 (62,63). Nous avons défini le critère rechute selon des paramètres ophtalmologiques objectifs, indépendamment de l'arrêt du traitement étudié. En cas de rechute sous immunosuppresseur conventionnel, même en cas de relais par un autre traitement du groupe, le patient était considéré comme ayant atteint le critère de jugement principal. 
Les critères de jugement secondaires étaient :

- Épargne cortisonique à 1 mois, 2 mois, 6 mois, 12 mois et 24 mois de traitement

- Évolution de l'acuité visuelle à 1 mois, 6 mois et 12 mois de traitement. Une amélioration était définie comme une amélioration de l'acuité visuelle d'au moins trois lignes selon l'échelle ETDRS. Une stabilité correspondait à une amélioration ou une dégradation de l'acuité visuelle $\leq 2$ lignes

- Évolution des paramètres d'inflammation intraoculaire à 1 mois, 6 mois et 12 mois de traitement. Un état de quiescence était défini comme une inflammation intraoculaire antérieure et vitréenne $\leq 0,5+$

- Effets indésirables observés au cours du traitement

\subsection{Recueil des données}

La collecte des données a été effectuée par une seule personne de façon rétrospective depuis le diagnostic de l'uvéite jusqu'à l'avènement du critère de jugement principal ou jusqu'à la date de la dernière consultation disponible (date de point). Après la survenue du critère de jugement principal, les données de suivi n'étaient plus recueillies.

\subsubsection{Données démographiques}

Pour chaque patient était recueilli :

- $\quad$ Age, le sexe et le poids

- Antécédents particuliers, notamment les antécédents pouvant être responsables d'atteinte oculaire (HTA, diabète)

- Étiologie de l'uvéite et les critères diagnostiques retenus

- Caractère uni- ou bilatéral de l'uvéite

- Localisation de l'uvéite : intermédiaire, postérieure ou panuvéite

\subsubsection{Données cliniques ophtalmologiques}

Pour chaque patient était relevé au diagnostic, lors de l'introduction du traitement immunosuppresseur conventionnel ou de la biothérapie, lors de la rechute sous traitement ou à la fin du suivi, les paramètres ophtalmologiques suivants :

- Acuité visuelle 
- Présence d'une inflammation intraoculaire, antérieure et vitréenne, estimée à travers les échelles du SUN

- Caractéristiques sémiologiques de l'uvéite : granulomateuse, présence de synéchie, hypertension intraoculaire

- Présence d'une cataracte

- Présence d'une vascularite active à l'angiographie

- Présence d'un œdème maculaire cystoïde, selon la définition sus-précisée

\subsubsection{Données thérapeutiques}

Pour chaque patient était recueilli :

- Date du début de traitement ophtalmologique spécifique et historique des lignes thérapeutiques précédant le traitement en cours lors de l'inclusion

- Durée de l'exposition cortisonique avant l'introduction du traitement en cours lors de l'inclusion

- Nom du traitement en cours lors de l'inclusion, sa posologie, le motif d'introduction et la date d'introduction en distinguant chaque patient en fonction de son groupe d'attribution (immunosuppresseur conventionnel versus biothérapie)

- Pour les patients du groupe biothérapie : la présence d'un traitement concomitant par immunosuppresseur conventionnel, son nom et sa posologie

- Posologie de corticoïdes au cours du suivi, ainsi qu'un éventuel recours aux bolus intraveineux

- Présence d'un traitement concomitant par collyres de corticoïdes, par collyres hypotonisants, par injection sous-conjonctivale ou sous-ténonienne de corticoïdes

\subsubsection{Données de fin de suivi}

Pour chaque patient était recueilli en fin de suivi :

- Traitement en cours à la fin du suivi, sa posologie

- Posologie de corticoïdes à la fin du suivi

- Statut clinique à la fin du suivi : stabilité, rechute, effet indésirable grave ayant nécessité l'arrêt du traitement, perdu de vue

\subsubsection{Données de tolérance}

Pour chaque patient était recueilli les effets indésirables thérapeutiques : 
- Infectieux : en précisant leurs gravités (sévère ayant nécessité une prise en charge en réanimation, modérée nécessitant une hospitalisation en médecine conventionnelle ou non grave prise en charge en ambulatoire). Les infections tuberculeuses étaient distinguées des autres infections

- Réactions allergiques systémiques ou au point d'injection en distinguant leur degré de gravité

- Troubles digestifs

- Manifestations auto-immunes paradoxales

- Néoplasie en précisant l'entité étiologique et le délai diagnostique

- Lésions démyélinisantes

- Syndrome dépressif

- Autres effets indésirables tels l'asthénie, les céphalées et les troubles du sommeil

\subsection{Analyses statistiques}

Les valeurs quantitatives ont été exprimées par la médiane et l'IQR (Interquartile range). Elles ont été analysées par le test non paramétrique de Mann-Whitney. Les valeurs qualitatives ont été exprimées en valeur absolue et en pourcentages. Elles ont été analysées par le test du Chi 2 quand l'effectif le permettait ou par le test exact de Fisher.

L'analyse de survie sans progression a été réalisée par la méthode de Kaplan-Meier, où l'évènement étudié était la rechute ou l'arrêt du traitement pour effet indésirable sévère. Les comparaisons de survie en fonction des traitements ont été réalisées grâce au test du Log Rank.

L'évolution des paramètres quantitatifs au cours du temps (acuité visuelle, épaisseur maculaire centrale de la rétine et posologie de corticoïdes) a été comparée grâce au test Anova.

La comparaison de la posologie de corticoïdes lors de la rechute sous immunosuppresseurs conventionnels et sous biothérapies chez un même patient a été effectuée grâce au $t$ test de Student pour données appariées.

L'analyse univariée des facteurs associés à la prescription de biothérapie a été réalisée par le calcul des odd-ratios.

Le seuil de significativité pour l'ensemble des tests était fixé à un $\mathrm{p}<0,05$.

Les analyses statistiques ont été réalisées grâce aux logiciels R Studio Version 1.0.153 et GraphPad Prism Version 8.1.2. 


\section{Résultats}

\subsection{Caractéristiques générales de la cohorte}

D'avril 2014 à septembre 2018, 1053 dossiers ont été extraits (Figure 11). Parmi ces patients, seuls 279 correspondaient aux critères d'inclusions.

Les causes d'exclusion des 774 patients restant étaient hétérogènes. Après lecture des dossiers médicaux, 41 dossiers étaient incomplets. Soixante-sept patients correspondaient à une population pédiatrique. Le codage d'uvéite était incorrect pour 101 patients incluant 37 cas de sclérites, 6 cas de neuropathies optiques et enfin, 58 cas de diagnostics divers (conjonctivite, syndrome sec...). De plus, 11 patients présentaient en réalité une pseudo-uvéite ou masquerade syndrome d'origine tumorale. L'uvéite était uniquement antérieure pour 330 patients, dont 90 patients présentant une uvéite antérieure associée à l'haplotype HLA B27. Deux-cent-un patients présentaient une uvéite d'étiologie infectieuse : 57 uvéites liées à une toxoplasmose, 52 uvéites tuberculeuses, 64 atteintes d'origine virale (38 uvéites à HSV et 26 uvéites à VZV), 9 uvéites syphilitiques et 19 uvéites d'autres origines infectieuses. Enfin, 23 patients souffraient d'une uvéite d'origine inflammatoire mais ne nécessitant pas de traitement spécifique (syndrome d'Irvin Gass, multiple evanescent white dot syndrome...).

Au sein des 279 patients recensés, seuls 75 patients ont été définitivement inclus. La raison principale d'exclusion des dossiers était thérapeutique. Cent-quatre-vingt-un patients recevaient uniquement des corticoïdes, sans autre traitement immunosuppresseur associé. Sept patients étaient traités par interféron alpha-2a. Enfin, 3 patients bénéficiaient d'un traitement immunosuppresseur conventionnel mais pour une indication extra-ophtalmologique et 13 dossiers étaient incomplets.

Un total de 75 patients a donc été inclus dans notre travail. Trente-six patients étaient traités par biothérapies et 39 par immunosuppresseurs conventionnels. 


\begin{tabular}{|c|}
\hline 1053 patients \\
extraits
\end{tabular}

Exclus

- 101 patients avec un diagnostic différentiel ophtalmologique (sclérite, conjonctivite...)

- 67 patients pédiatriques

- 330 uvéites antérieures

- 201 uvéites infectieuses

- 11 lymphomes oculaires

- 23 autres étiologies d'uvéites ne nécessitant pas de traitement anti inflammatoire (uvéite d'Irvin Gass, MEWDS...)

- 41 dossiers incomplets

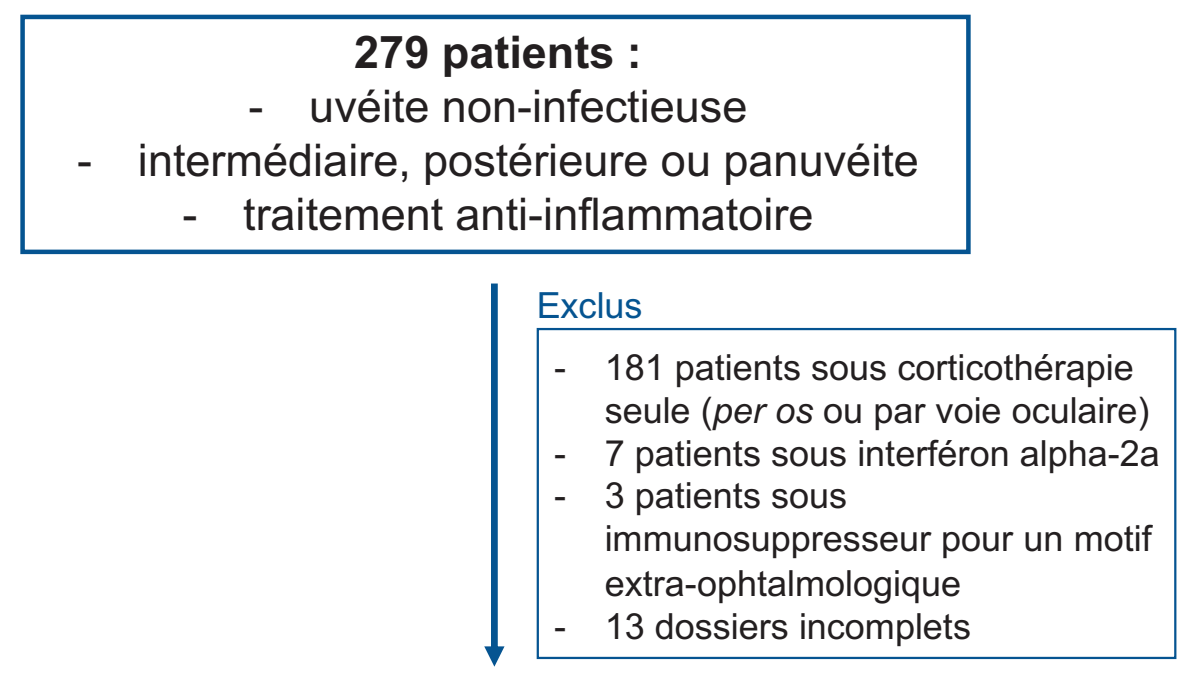

\section{5 patients inclus}
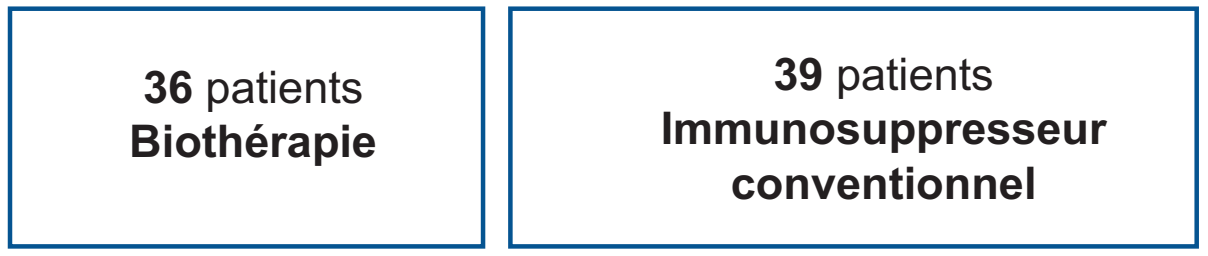

Figure 11 : Diagramme de flux. MEDWS : Multiple evanescent white dot syndrome. 


\subsection{Description du groupe biothérapie}

3.2.1. Caractéristiques générales et ophtalmologiques lors de l'introduction du $\underline{\text { traitement }}$

Trente-six patients ont pu être inclus dans le groupe biothérapie. L'âge médian (IQR) à l'introduction du traitement était de 43 ans [32 - 62]. Les patients étaient de sexe masculin dans $61 \%$ des cas. Ils présentaient peu de comorbidités influençant la prise en charge thérapeutique et responsables d'atteintes oculaires (6 patients avaient une HTA et aucun patient n'était diabétique).

Ces uvéites étaient rattachées à un large panel étiologique, la majorité d'origine idiopathique (33\%). L'étiologie la plus fréquemment retrouvée était la maladie de birdshot (9 patients, soit $25 \%$ ), suivie de la maladie de Behçet (7 patients, soit $20 \%$ ). Deux autres étiologies étaient remarquables : 3 cas de sarcoïdose $(8 \%)$ et 3 cas de choroïdite multifocale $(8 \%)$. Un cas associé à une maladie de Crohn et un cas associé à un rhumatisme psoriasique ont été inclus.

Il s'agissait presque exclusivement d'atteinte bilatérale (97\%). Pour la moitié des patients, l'uvéite était localisée au segment postérieur, suivie des panuvéites pour 16 malades (44\%) et enfin seuls 2 patients (6\%) avaient une uvéite intermédiaire. Lors de l'introduction de la biothérapie, l'acuité visuelle médiane était de 0,35 LogMAR [0 - 0,5] pour l'œil droit et $0,15[0,025-0,50]$ pour l'œil gauche. Les uvéites étaient sévères puisque plus de la moitié des patients à l'inclusion présentaient un œdème maculaire, confirmé à l'OCT (20 patients, soit $56 \%$ ). Chez 13 patients (36\%) l'angiographie retrouvait des signes de vascularite active. La moitié des patients présentaient une inflammation du segment antérieur et $66 \%$ une atteinte inflammatoire du vitré. Cette inflammation restait modérée avec un Tyndall à 0,5 et $1+$ chez respectivement $20 \%$ des patients. De même, l'atteinte inflammatoire du vitré s'avérait elle aussi modérée, évaluée à $0,5+(17 \%)$ ou $1+(40 \%)$.

\subsubsection{Prise en charge thérapeutique}

La durée médiane de suivi était de 9,5 mois [4 - 22]. Par ailleurs, la durée médiane d'évolution de l'uvéite était de 16 mois lors de l'initiation du traitement [9-66].

L'ensemble des patients étaient, selon la définition du groupe, traités par biothérapies. La majorité des patients (94\%) étaient traités par anti-TNF alpha. L'adalimumab (Humira®), 
seul anti-TNF alpha ayant reçu l'AMM, représentait le traitement le plus fréquemment utilisé (19 patients, soit $53 \%$ ). Quinze patients, soit $41 \%$, bénéficiaient d'infliximab (Inflectra ${ }^{\circledR}$ pour un patient et Remicade ${ }^{\circledR}$ pour les autres malades). Seuls 2 patients $(6 \%)$ ont été traités par tocilizumab (Roactemra $\left.{ }^{\circledR}\right)$. Le schéma d'administration de ces molécules était variable. L'ensemble des patients traités par infliximab recevait une posologie de $5 \mathrm{mg} / \mathrm{kg}$ à $\mathrm{J} 0$ puis $\mathrm{J} 15$. Puis, un tiers des patients recevaient l'injection suivante à 4 semaines, un tiers à 6 semaines et un tiers à 8 semaines. Seul un patient traité par adalimumab a reçu une dose de charge de $80 \mathrm{mg}$. L'adalimumab était administré en une injection sous-cutanée de 40 mg toutes les deux semaines. Enfin, les deux patients traités par tocilizumab ont reçu le traitement par voie sous-cutanée à la posologie de $162 \mathrm{mg}$ hebdomadaire. Cinq patients (14\%) recevaient de manière concomitante un traitement par méthotrexate et 2 patients $(6 \%)$ un traitement par azathioprine.

Trente-et-un pour cent des patients n'avaient pas reçu de traitement immunosuppresseur conventionnel avant l'introduction de la biothérapie, $41 \%$ avaient reçu une ligne thérapeutique antérieure et enfin $28 \%$ avaient précédemment bénéficié de deux lignes thérapeutiques n'ayant pas permis de contrôler efficacement l'inflammation oculaire. Lorsqu'ils avaient bénéficié d'un traitement immunosuppresseur conventionnel avant la biothérapie, le traitement choisi était l'azathioprine dans $31 \%$ des cas, suivi du mycophénolate mofétil (25\%), du méthotrexate $(22 \%)$ et enfin de l'interféron alpha-2a (19\%).

La durée médiane d'exposition à la corticothérapie avant l'introduction de la biothérapie était de 13 mois [7 - 62]. La moitié des patients présentait une cortico-dépendance de plus de $20 \mathrm{mg} /$ jour de prednisone et $19 \%$ une cortico-dépendance supérieure à $35 \mathrm{mg} /$ jour. Lors de l'initiation de la biothérapie, la posologie médiane (IQR) quotidienne de corticoïdes (prednisone, Cortancyl® dans $100 \%$ des cas) était de 30 mg [20 - 49] (Figure 12). Après un mois de traitement, celle-ci diminuait à $28 \mathrm{mg}$ [16 - 40], puis à $20 \mathrm{mg}$ [13 - 30] après deux mois d'administration. A 6 mois $(n=25)$, la posologie médiane journalière de corticoïdes était de $11 \mathrm{mg}$ [7 - 17]. A un an de l'introduction de la biothérapie $(\mathrm{n}=17)$, la posologie médiane de corticoïdes était de $7 \mathrm{mg}$ [5 - 10], soit aux objectifs d'épargne cortisonique ( $\leq 10 \mathrm{mg} / \mathrm{jour}$ ). Après deux ans de traitement $(\mathrm{n}=7)$, la posologie médiane de corticoïdes était inférieure à $5 \mathrm{mg}$ (2,5 mg [0 - 9]). La décroissance de la corticothérapie au cours du temps, passant d'une posologie initiale médiane de $30 \mathrm{mg}$ à $2,5 \mathrm{mg}$, était statistiquement significative $(\mathrm{p}<0,0001)$. A 12 mois de traitement, $83 \%$ des patients recevaient une dose quotidienne de 
corticoïdes $\leq 10 \mathrm{mg}$ et $44 \%$ une dose $\leq 5 \mathrm{mg}$. Huit patients $(22 \%)$ ont pu être sevrés en corticoïdes sur l'ensemble du suivi.

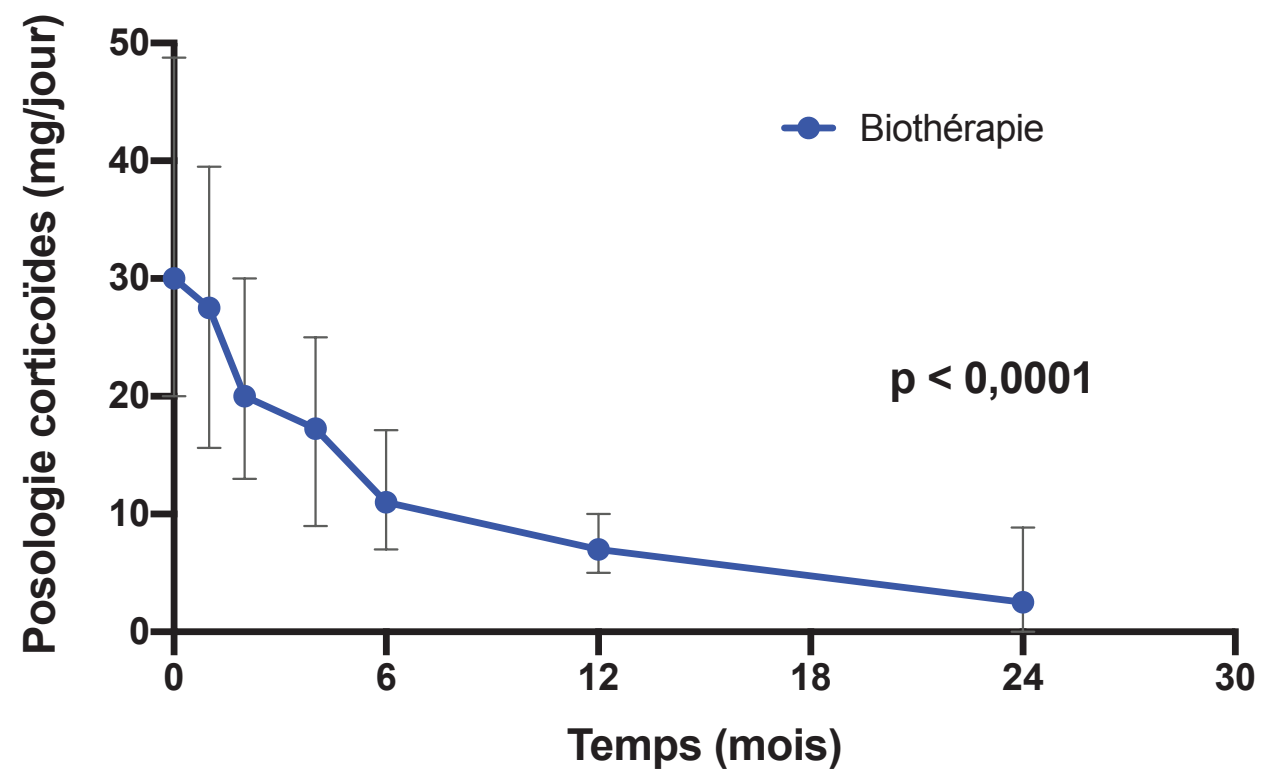

Figure 12 : Évolution de la posologie médiane quotidienne de prednisone (mg/jour) au cours du temps chez les patients traités par biothérapies. Représentation graphique des médianes et IQR (interquartile range). 


\subsubsection{Analyse du critère de jugement principal}

Vingt patients, soit $56 \%$ des patients traités par biothérapies, ont atteint le critère de jugement principal : 15 patients (41\%) ont présenté une rechute et 5 patients (14\%) ont subi un effet indésirable sévère motivant l'arrêt du traitement. Un seul malade a été perdu de vue.

Le délai médian de survenue du critère de jugement principal (Figure 13) était de 16 mois. Après 6 mois de traitement, 70,4 \% des patients étaient indemnes de rechute ou d'effet indésirable nécessitant l'arrêt du traitement (taux de survie sans progression). Après un an de traitement, 60,4 \% des patients n'avaient pas présenté le critère de jugement principal. A deux ans de traitement, ce taux de survie sans progression s'élevait à 43,7\% des patients.

L'analyse isolée du taux de rechute a montré que 15 patients ont présenté une rechute (41\%) et le délai médian de rechute était de 29 mois (Figure 14). La posologie médiane de corticoïdes lors de la rechute était de 9,5 mg [5,75-20]. Lors de la rechute, les patients ont été pris en charge de la manière suivante : un rapprochement des cures d'infliximab (2 patients) ou des injections d'adalimumab (2 patients), un changement de traitement pour l'infliximab pour 3 patients traités par adalimumab, un changement de traitement pour le tocilizumab (2 patients sous adalimumab et un patient traité par infliximab), un relais du tocilizumab sous-cutané au tocilizumab intra-veineux, un changement de traitement pour l'interféron alpha-2a (2 patients sous adalimumab), une majoration de la posologie de corticoïdes per os isolée sans changement de biothérapie (adalimumab) et la réalisation d'une injection sous-conjonctivale de corticoïdes sans changement de biothérapie (adalimumab). Deux patients ont rechuté lors de l'espacement des cures d'infliximab.

\subsection{4. Évolution des paramètres ophtalmologiques sous traitement}

Après un an de traitement $(\mathrm{n}=17)$, l'acuité visuelle (LogMAR) médiane (IQR) était de $0,075[0-0,50]$ pour l'œil droit et de 0,1 [0,025-0,26] pour l'œil gauche (Figure 15.A). Cette progression de l'acuité visuelle sous traitement était à la limite de la significativité ( $p=0,07$ et $\mathrm{p}=0,05$ pour l'œil droit et l'œil gauche respectivement). Après un mois de traitement $(\mathrm{n}=33)$, une amélioration (gain $\geq 3$ lignes) de l'acuité visuelle de l'œil droit était constatée chez $18 \%$ des patients et une stabilité chez $82 \%$. A 6 mois de traitement $(n=25), 12 \%$ des patients présentaient une amélioration de leur acuité visuelle, $76 \%$ une stabilité et $12 \%$ une dégradation de celle-ci (Figure 15.C). 


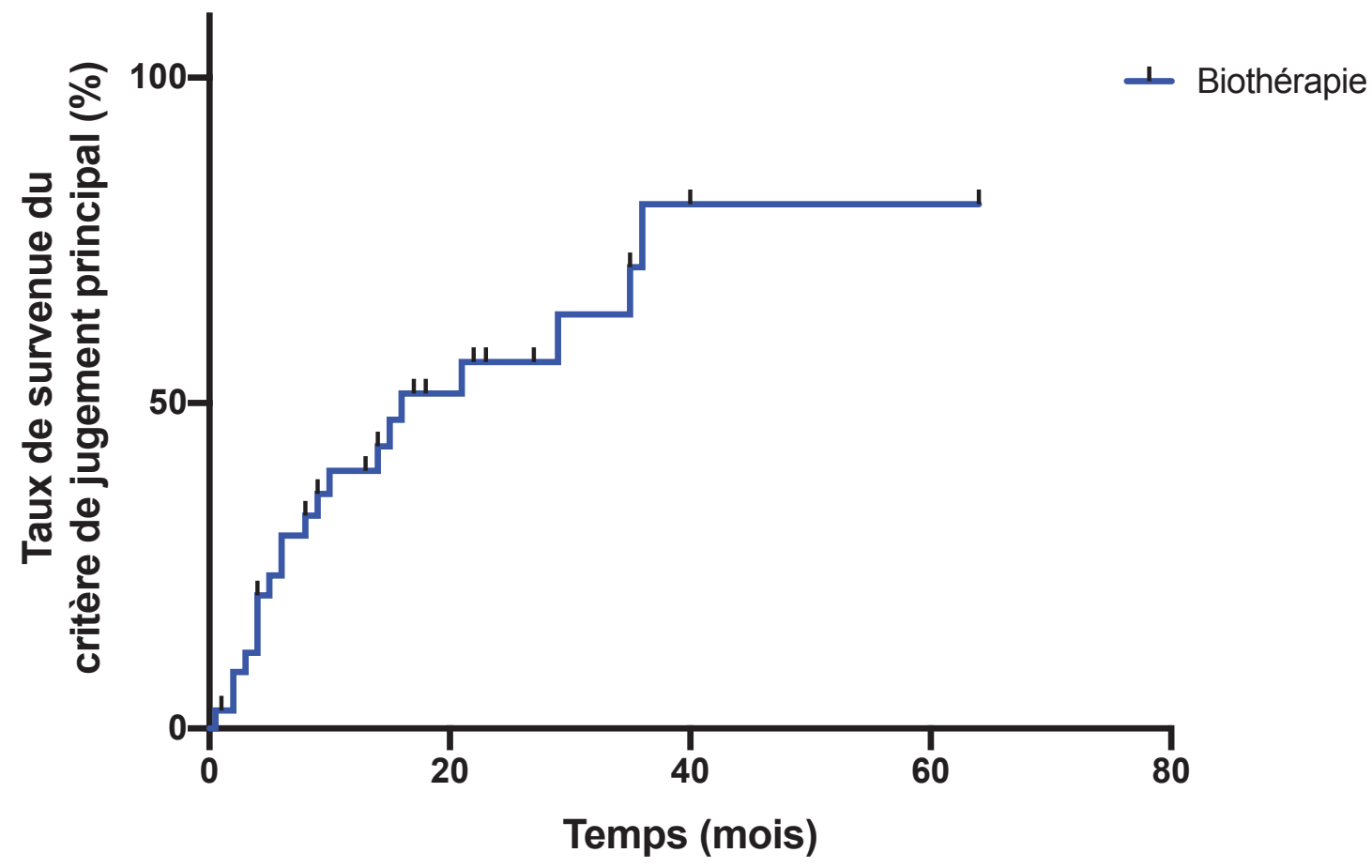

Figure 13 : Courbe de Kaplan-Meier représentant la survenue du critère de jugement principal lors du traitement par biothérapies. Les tirets verticaux représentent les données censurées.

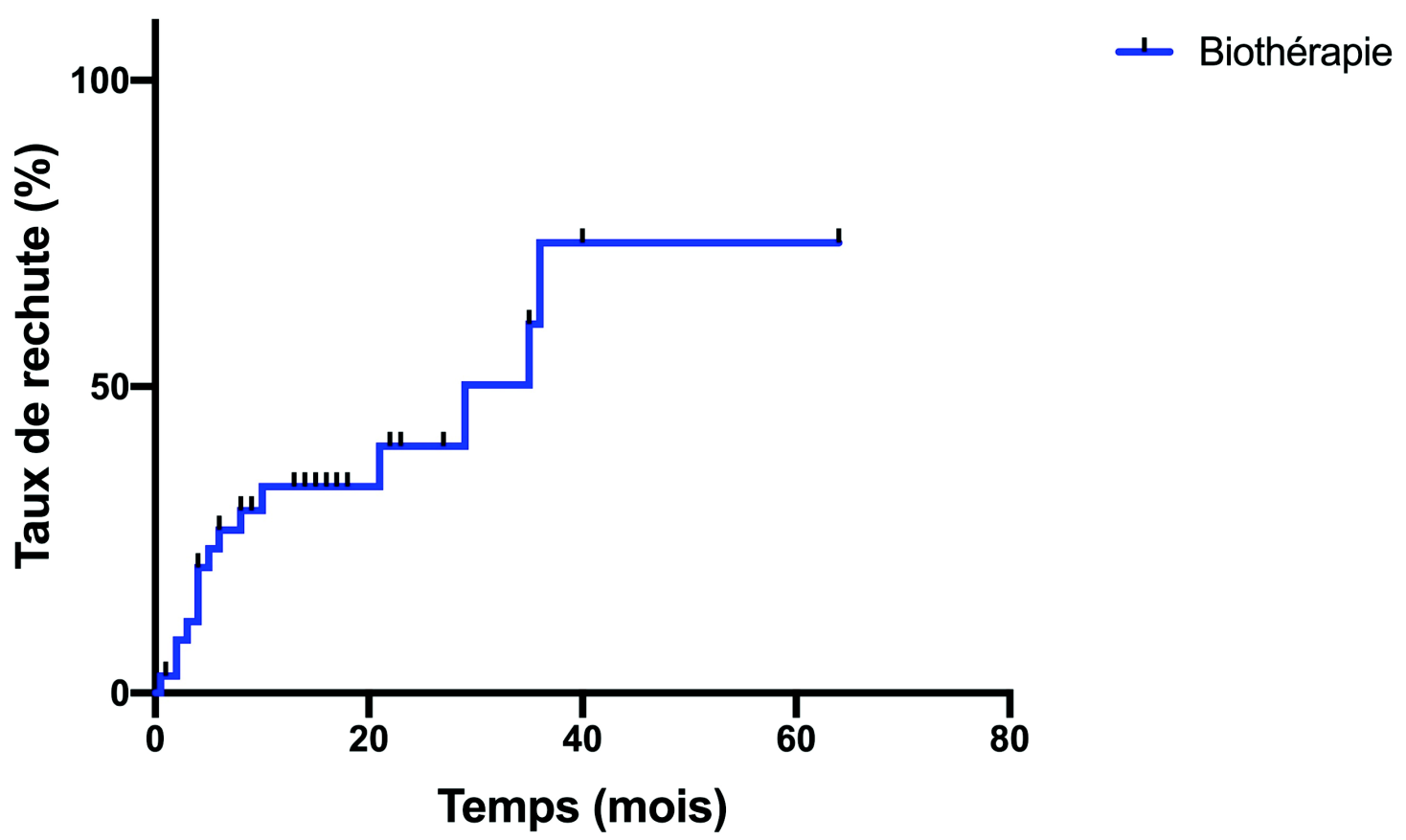

Figure 14 : Courbe de Kaplan-Meier représentant la survenue d'une rechute lors du traitement par biothérapies. Les tirets verticaux représentent les données censurées. 
A 12 mois de traitement $(n=17)$, une amélioration de l'acuité visuelle était relevée dans $25 \%$ des cas, une stabilité dans $69 \%$ et une dégradation dans $6 \%$. Concernant l'œil gauche (Figure 15.D), une amélioration était visible chez $13 \%$ des patients et une stabilité chez $87 \%$ après un mois de traitement. A 6 mois de traitement, une amélioration a été notée chez $7 \%$ des patients, une stabilité pour $81 \%$, une dégradation pour $12 \%$ des patients. A 12 mois de traitement, une amélioration de l'acuité visuelle sous traitement était observée chez $19 \%$ des patients, une stabilité pour $75 \%$ et une dégradation pour $6 \%$ des malades.

Concernant l'efficacité du traitement sur l'inflammation intraoculaire, à l'introduction de la biothérapie, $41 \%$ des patients présentaient un état de quiescence oculaire. Ce taux s'élevait à $76 \%$ à un mois, $92 \%$ à 6 mois et $71 \%$ à 12 mois.

Concernant l'évolution de l'œdème maculaire cystoïde, la médiane d'épaisseur maculaire centrale (IQR), mesurée en OCT, à l'introduction du traitement était de $433 \mu \mathrm{m}$ [323 -555] pour l'œil droit et $342 \mu \mathrm{m}$ [317 - 404] pour l'œil gauche. Après un mois de traitement, ces chiffres étaient respectivement de $324 \mu \mathrm{m}$ [293 - 525] pour l'œil droit et $315 \mu \mathrm{m}$ [294 - 392] pour l'œil gauche (Figure 15.B). Après 6 mois de traitement, on observait une normalisation de l'épaisseur maculaire avec des valeurs de $290 \mu \mathrm{m}$ [274 - 302] pour l'œil droit et de $254 \mu \mathrm{m}$ [229 - 304] pour l'œil gauche. Après un an de traitement, ces chiffres étaient de $286 \mu \mathrm{m}$ [252 - 315] pour l'œil droit et $285 \mu \mathrm{m}$ [228 - 335] pour l'œil gauche. Cette évolution au cours du temps était à la limite de la significativité ( $\mathrm{p}=0,05$ pour les deux yeux).

Vingt patients (56 \%) présentaient un œdème maculaire cystoïde au diagnostic. La moitié des patients $(\mathrm{n}=10)$ ont présenté une correction de l'œdème maculaire sous traitement. Parmi ces 10 patients, 2 ont été considérés comme ayant présenté une rechute sur d'autres paramètres que l'œdème maculaire. Neuf des 10 patients pour lesquels le traitement n'a pas permis une amélioration de l'œdème maculaire ont été considérés comme ayant souffert d'une rechute. Seul un patient a poursuivi le traitement à l'identique, malgré la présence de l'œdème maculaire, devant une franche amélioration de l'épaisseur maculaire centrale en OCT et de l'acuité visuelle.

Enfin, 13 patients présentaient une vascularite active (36\%) lors de l'initiation du traitement. Sous biothérapies, une résolution de la vascularite a été obtenue chez $69 \%$ des patients ( 9 patients). Parmi ces 9 patients, un seul a été considéré comme ayant présenté une rechute sur d'autres paramètres que la vascularite. Parmi les 4 patients n'ayant pas eu d'amélioration de la vascularite sous traitement, seuls deux ont été considérés comme présentant une rechute devant une amélioration insuffisante des lésions vasculaires. 

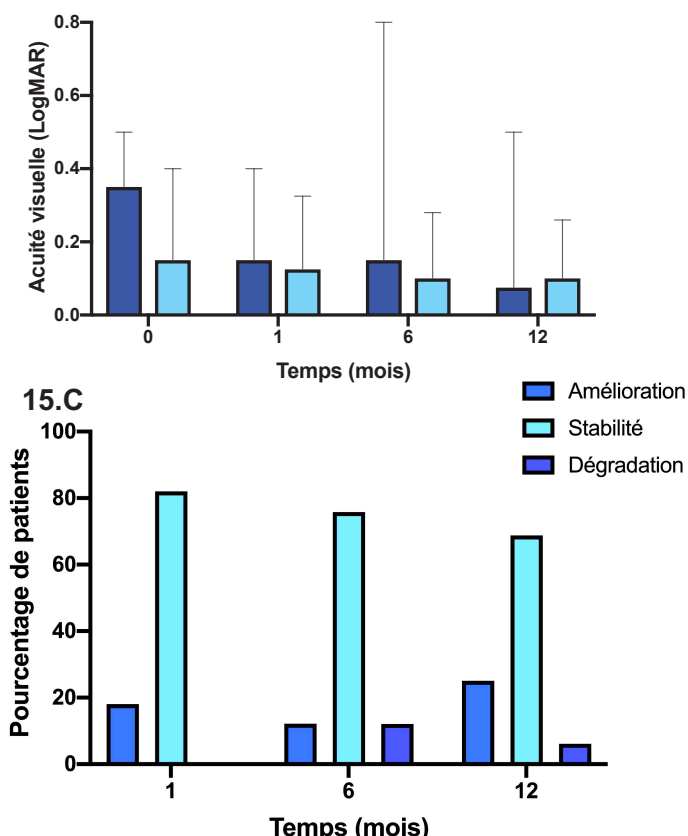

15.B

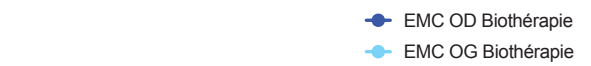

15.D
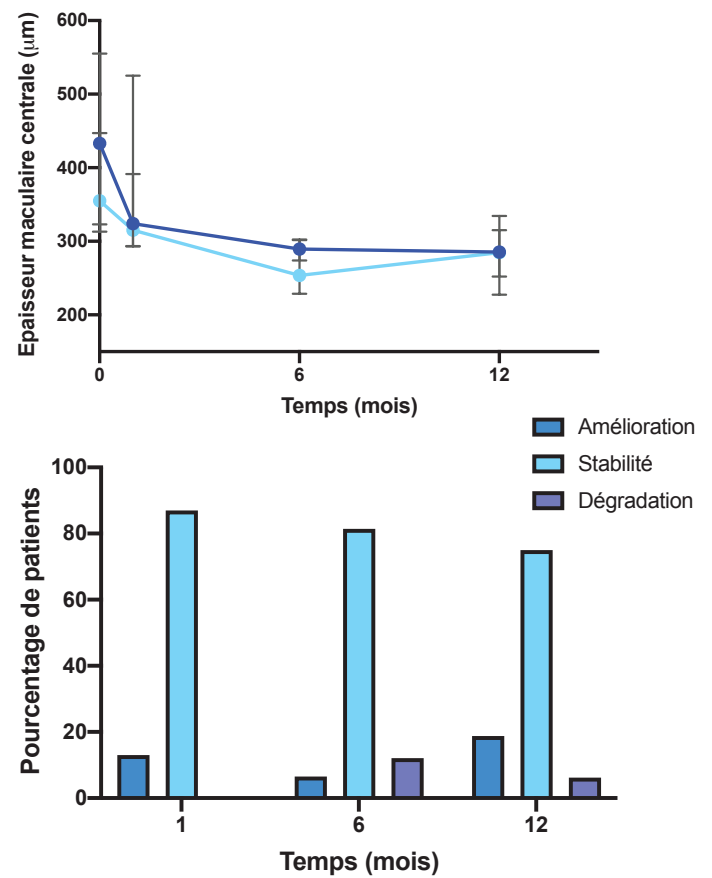

Figure 15 : Évolution de l'acuité visuelle (AV) (15.A) et de l'épaisseur maculaire centrale (EMC) (15.B) au cours des 12 premiers mois de traitement par biothérapies. Évolution de l'acuité visuelle au cours des 12 premiers mois selon les critères d'amélioration, de stabilité ou de dégradation pour l'œil droit (15.C) et l'œil gauche (15.D). OD : œil droit. OG : œil gauche. Représentation graphique des médianes et IQR (interquartile range). 


\subsubsection{Caractéristiques à la fin du suivi}

Parmi les 36 patients du groupe biothérapie, 16 patients (44\%) n'ont pas présenté de rechute ou d'effet indésirable grave au cours du suivi. La durée médiane du suivi était de 16 mois [8 -26]. A la fin de cette période, on observait une franche amélioration de la fonction visuelle, avec une acuité visuelle respectivement de 0,13 LogMAR [0 - 0,28] pour l'œil droit et $0[0-0,10]$ pour l'œil gauche. Une vascularite active et une cataracte étaient observées dans $13 \%$ des cas. Enfin, seul un patient présentait un œdème maculaire. La posologie médiane quotidienne de prednisone à la fin du suivi pour ces 16 malades était de $5 \mathrm{mg} /$ jour $[0-9,8]$.

Parmi ces 16 malades n'ayant pas présenté le critère de jugement principal, 6 patients $(38 \%)$ ont pu arrêter le traitement par biothérapies. Ces 6 patients étaient tous traités par infliximab. Quatre patients avaient une maladie de Behçet, un patient une uvéite idiopathique et le dernier une uvéite associée à un rhumatisme psoriasique. Un seul a présenté une rechute 12 mois après l'arrêt du traitement.

\subsubsection{Tolérance et effets indésirables}

La moitié des patients ont présenté au moins un effet indésirable au cours du suivi. Un total de 31 effets indésirables a été recensé. Devant la sévérité de cinq de ces réactions, le traitement a été arrêté. Ces réactions étaient : rectorragies importantes, apparition d'un psoriasis bulleux sévère, développement d'un syndrome dépressif, réaction anaphylactique sévère (sous infliximab) et développement d'un syndrome dépressif sous adalimumab.

Onze patients $(31 \%)$ ont développé au moins une infection pendant la période de traitement. Aucune de ces infections n'a nécessité une hospitalisation. Il s'agissait d'infections ORL ou bronchique pour 7 patients, d'infections uro-génitales pour 2 patients et d'infections digestives virales pour 2 patients.

Parmi les autres effets indésirables notables, 2 patients $(6 \%)$ ont développé une néoplasie au cours du suivi. La première patiente a développé une néoplasie cervicale utérine de grade I, d'évolution favorable après prise en charge chirurgicale, survenu deux ans après l'arrêt de l'infliximab. Le deuxième patient a développé un cancer pulmonaire d'emblée métastatique, 6 mois après le début du traitement par tocilizumab, dans un contexte d'exposition antérieure à l'azathioprine pendant 9 mois. Ce traitement par tocilizumab a été arrêté dès le diagnostic. Le patient est décédé 5 mois après le diagnostic. Un patient a également développé un rhumatisme psoriasique et cutané, lors de l'espacement des cures d'infliximab. Le psoriasis a nécessité l'ajout de méthotrexate. 


\subsubsection{Analyse de sous-groupes selon le type de biothérapie reçu}

Les étiologies les plus représentées chez les patients traités par infliximab étaient la maladie de Behçet (33\%) et les uvéites idiopathiques (33\%). Sept patients (37\%) sous adalimumab souffraient d'une maladie de birdshot et 6 (32\%) d'une uvéite idiopathique (Tableau 7).

Les patients sous tocilizumab semblaient présenter une uvéite plus sévère avec une vascularite active et un œdème maculaire, même si l'acuité visuelle était préservée. L'acuité visuelle était plus altérée chez les patients sous infliximab $(0,50[0,050-1,1])$.

Le critère de jugement principal a été atteint chez $63 \%$ des patients sous adalimumab, $47 \%$ des patients sous infliximab, et pour un des deux patients sous tocilizumab $(p=0,49)$. De plus, $58 \%$ des patients sous adalimumab ont présenté une rechute contre seulement $20 \%$ des patients sous infliximab $(\mathrm{p}=0,04)$. Par ailleurs, $27 \%$ des patients sous infliximab ont souffert d'un effet indésirable sévère contre $5 \%$ des patients recevant de l'adalimumab $(p=0,15)$. 


\begin{tabular}{|c|c|c|c|c|}
\hline & Adalimumab & Infliximab & Tocilizumab & $\mathbf{p}$ \\
\hline Effectif (valeur absolue et \%) & $19(53)$ & $15(41)$ & $2(6)$ & \\
\hline \multicolumn{5}{|l|}{ Caractéristiques générales de la population } \\
\hline Age median [IQR] & $46[33-62]$ & $41[28-60]$ & $51[32-69]$ & \\
\hline Sexe : homme & $11(58)$ & $9(60)$ & $2(100)$ & \\
\hline \multicolumn{5}{|l|}{ Etiologie de l'uvéite } \\
\hline Idiopathique & $6(32)$ & $5(33)$ & $1(50)$ & \\
\hline Maladie de birdshot & $7(37)$ & $1(8)$ & $1(50)$ & \\
\hline Maladie de Behçet & $2(10)$ & $5(33)$ & 0 & \\
\hline Choroïdite multi-focale & $3(16)$ & 0 & 0 & \\
\hline Sarcoïdose & $1(5)$ & $2(13)$ & 0 & \\
\hline Vogt-Koyanagi-Harada & 0 & 0 & 0 & \\
\hline Autres & 0 & $2(13)$ & 0 & \\
\hline \multicolumn{5}{|l|}{ Caractéristiques ophtalmologiques initiales } \\
\hline Bilatéralité & $18(95)$ & $15(100)$ & $2(100)$ & \\
\hline \multicolumn{5}{|l|}{ Localisation uvéite } \\
\hline intermédiaire & 0 & $1(7)$ & $1(50)$ & \\
\hline postérieure & $14(74)$ & $3(20)$ & $1(50)$ & \\
\hline panuvéite & $5(26)$ & $11(73)$ & 0 & \\
\hline $\begin{array}{l}\text { Acuité visuelle médiane (LogMAR) de l'œil } \\
\text { droit (OD) [IQR] }\end{array}$ & $\begin{array}{c}0,20 \\
{[0-0,50]}\end{array}$ & $\begin{array}{c}0,50 \\
{[0,050-1,1]}\end{array}$ & $\begin{array}{c}0,13 \\
{[0,10-0,15]}\end{array}$ & \\
\hline $\begin{array}{l}\text { Acuité visuelle médiane (LogMAR) de l'œil } \\
\text { gauche (OG) [IQR] }\end{array}$ & $\begin{array}{c}0,19 \\
{[0-0,48]}\end{array}$ & $\begin{array}{c}0,19 \\
{[0,075-0,80]}\end{array}$ & $\begin{array}{c}0,075 \\
{[0,050-0,1]}\end{array}$ & \\
\hline Uvéite granulomateuse & $1(5)$ & $1(8)$ & 0 & \\
\hline Vascularite active & $7(37)$ & $4(27)$ & $2(100)$ & \\
\hline OEdème maculaire cystoïde & $12(63)$ & $6(40)$ & $2(100)$ & \\
\hline $\begin{array}{l}\text { épaisseur maculaire centrale médiane }(\mu \mathrm{m} \\
\text { en OCT) OD [IQR] }\end{array}$ & $\begin{array}{c}446 \\
{[343-542]}\end{array}$ & $\begin{array}{c}331 \\
{[280-624]}\end{array}$ & $\begin{array}{c}313 \\
{[313-313]}\end{array}$ & \\
\hline $\begin{array}{l}\text { épaisseur maculaire centrale médiane ( } \mu \mathrm{m} \\
\text { en OCT) OG [IQR] }\end{array}$ & $\begin{array}{c}355 \\
{[317-495]}\end{array}$ & $\begin{array}{c}380 \\
{[322-438]}\end{array}$ & $\begin{array}{c}314 \\
{[303-325]}\end{array}$ & \\
\hline \multicolumn{5}{|l|}{ Prise en charge thérapeutique } \\
\hline $\begin{array}{l}\text { Durée médiane (mois) d'évolution de l'uvéite } \\
\text { [IQR] }\end{array}$ & $\begin{array}{c}13 \\
{[9-39]}\end{array}$ & $\begin{array}{c}54 \\
{[5-107]}\end{array}$ & $\begin{array}{c}66 \\
{[34-97]}\end{array}$ & \\
\hline $\begin{array}{l}\text { Durée médiane (mois) d'exposition antérieure } \\
\text { au traitement à la corticothérapie [IQR] }\end{array}$ & $\begin{array}{c}12 \\
{[5-26]}\end{array}$ & $\begin{array}{c}31 \\
{[5-100]}\end{array}$ & $\begin{array}{c}65 \\
{[32-97]}\end{array}$ & \\
\hline \multicolumn{5}{|l|}{ Critère de jugement principal } \\
\hline $\begin{array}{l}\text { Nombre de patients ayant atteint le critère de } \\
\text { jugement principal }\end{array}$ & $12(63)$ & $7(47)$ & $1(50)$ & 0,49 \\
\hline rechute & $11(58)$ & $3(20)$ & $1(50)$ & 0,04 \\
\hline effet indésirable sévère & $1(5)$ & $4(27)$ & 0 & 0,15 \\
\hline
\end{tabular}

Tableau 7 : Analyse des caractéristiques ophtalmologiques, de la prise en charge thérapeutique et du critère de jugement principal selon le type de biothérapie reçu. Les données qualitatives sont exprimées en valeur absolue et en pourcentages. Les données quantitatives sont exprimées en médiane et IQR. IQR : interquartile range. OD : œil droit. OG : œil gauche. OCT : tomographie par cohérence optique. 


\subsection{Description du groupe immunosuppresseur conventionnel}

3.3.1. Caractéristiques générales et ophtalmologiques lors de l'introduction du $\underline{\text { traitement }}$

Trente-neuf patients ont été inclus dans le groupe immunosuppresseur conventionnel. Trente-huit pour cent des patients était des hommes. L'âge médian (IQR) à l'introduction du traitement était de 49 ans [34 - 61]. Certains patients présentaient des comorbidités pouvant être responsables d'atteinte ophtalmologique : 8 avaient une HTA et 3 souffraient d'un diabète.

Sur le plan étiologique, les deux diagnostics principaux, représentant respectivement $28 \%$ et $26 \%$ des étiologies, étaient les uvéites idiopathiques et la maladie de birdshot. Le troisième diagnostic en termes de fréquence était la sarcoïdose, touchant 9 malades $(23 \%)$. On notait également 4 cas de maladies de Vogt-Koyanagi-Harada et 3 cas de maladie de Behçet. Enfin, on a pu relever une atteinte oculaire en lien avec un lupus érythémateux systémique et un cas d'ophtalmie sympathique.

Sur le plan ophtalmologique, $92 \%$ des patients présentaient une atteinte bilatérale. L'atteinte inflammatoire concernait l'ensemble des segments oculaires dans $56 \%$ des cas, le segment postérieur pour $39 \%$ des malades et le segment intermédiaire dans $5 \%$. L'acuité visuelle (LogMAR) médiane (IQR) à l'introduction du traitement immunosuppresseur était de $0,15[0,1-0,45]$ pour l'œil droit et de 0,22 [0,10-0,50] pour l'œil gauche. Il s'agissait d'uvéites sévères puisque $36 \%$ des patients avaient des stigmates d'inflammation vasculaire rétinienne et $59 \%$ présentaient un œdème maculaire cystoïde en OCT. Enfin, 10 patients $(27 \%)$ présentaient un Tyndall protéique $\geq 1+$ et 13 (35\%) une inflammation vitréenne $\geq 1+$.

\subsubsection{Prise en charge thérapeutique}

La durée médiane de suivi était de 13 mois [4 - 37] et la durée médiane d'évolution de l'uvéite antérieurement au début du traitement était de 11 mois [4 - 27,5].

Le nombre de patients traités par chaque immunosuppresseur était homogène puisque 15 patients (38\%) étaient traités par azathioprine, 14 (36\%) par méthotrexate et enfin, 10 (26\%) par mycophénolate mofétil. 


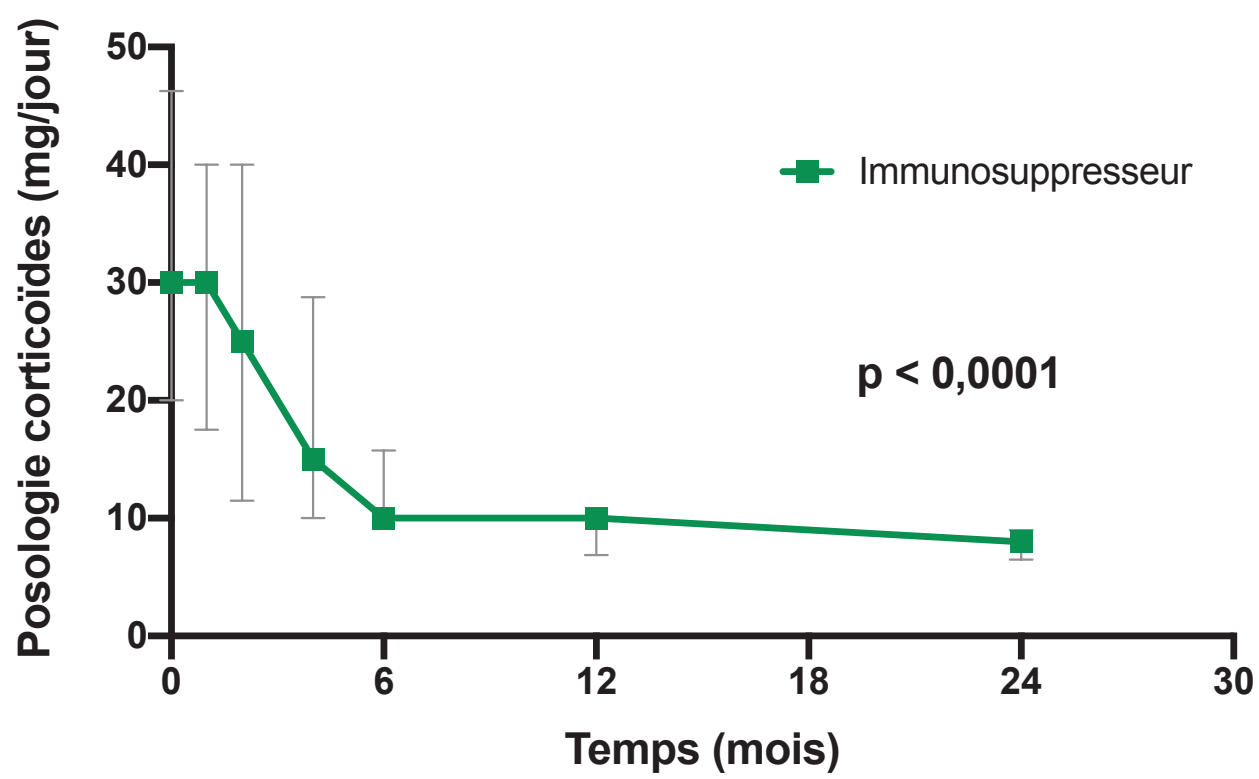

Figure 16 : Évolution de la posologie médiane quotidienne de prednisone (mg/jour) au cours du temps chez les patients traités par immunosuppresseurs conventionnels. Représentation graphique des médianes et IQR (interquartile range). 
Les posologies d'azathioprine étaient progressivement augmentées jusqu'à la dose de 100 à $150 \mathrm{mg} / \mathrm{jour}$, en fonction du poids des malades. De même, la posologie de méthotrexate variait entre $7,5 \mathrm{mg} / \mathrm{semaine}$ et $20 \mathrm{mg} / \mathrm{semaine}$, adaptée au poids du patient sur la base d'une prescription de $0,3 \mathrm{mg} / \mathrm{kg} / \mathrm{semaine}$. Enfin, les 10 patients traités par mycophénolate mofétil recevaient une dose de 1 gramme, deux fois par jour. En cohérence avec la définition du groupe, aucun patient n'avait reçu de traitement immunosuppresseur conventionnel ou de biothérapie antérieurement.

La durée médiane d'exposition antérieure à la corticothérapie était de 8 mois [3 - 22,5]. Trente-et-un pour cent des patients présentaient une cortico-dépendance de plus de $20 \mathrm{mg} / \mathrm{jour}$ de prednisone et $15 \%$ une cortico-dépendance supérieure à $35 \mathrm{mg} /$ jour. L'ensemble des patients recevaient un traitement concomitant par prednisone $\left(\right.$ Cortancyl $\left.{ }^{\circledR}\right)$. A l'introduction du traitement, la posologie médiane (IQR) de prednisone quotidienne était de $30 \mathrm{mg}$ [20 - 46]. Une décroissance de la corticothérapie a pu être observée au cours du temps (Figure 16). En effet, après deux mois de traitement, la posologie médiane était de $25 \mathrm{mg} /$ jour [12 - 40] puis de $10 \mathrm{mg} /$ jour $[10$ - 16] à 6 mois $(\mathrm{n}=29)$. On observait une stagnation de la posologie médiane de corticoïdes à $10 \mathrm{mg}[7-11]$ après un an de traitement $(\mathrm{n}=21)$. Enfin, après 2 ans de suivi $(\mathrm{n}=12)$, la posologie médiane de prednisone atteignait $8 \mathrm{mg}$ [7 - 9]. La décroissance de la corticothérapie au cours du temps, passant d'une posologie initiale médiane de $30 \mathrm{mg}$ à $8 \mathrm{mg}$, était statistiquement significative $(\mathrm{p}<0,0001)$ (Figure 16). A 12 mois de traitement, la posologie de corticoïdes était $\leq 10 \mathrm{mg} /$ jour pour $78 \%$ des patients et $\leq 5 \mathrm{mg} /$ jour pour $22 \%$. Sur l'ensemble du suivi, 5 patients (13\%) ont pu être sevrés en corticoïdes.

\subsubsection{Analyse du critère de jugement principal}

La survenue du critère de jugement principal a été observée chez 23 patients (59\%) : 19 patients ont présenté une rechute $(49 \%)$ et 4 patients $(10 \%)$ ont développé des effets indésirables sévères ayant nécessité d'interrompre le traitement. Durant l'ensemble du suivi, deux patients ont été perdus de vue.

Le délai médian de survenue du critère de jugement principal était de 21 mois (Figure 17). Après un mois de traitement, 89,7\% des patients étaient indemnes de toute rechute ou d'effet indésirable grave. Ce taux de survie sans progression diminuait à 71,7\% après 6 mois, $60,3 \%$ après un an et $46,5 \%$ après deux ans de traitement. 


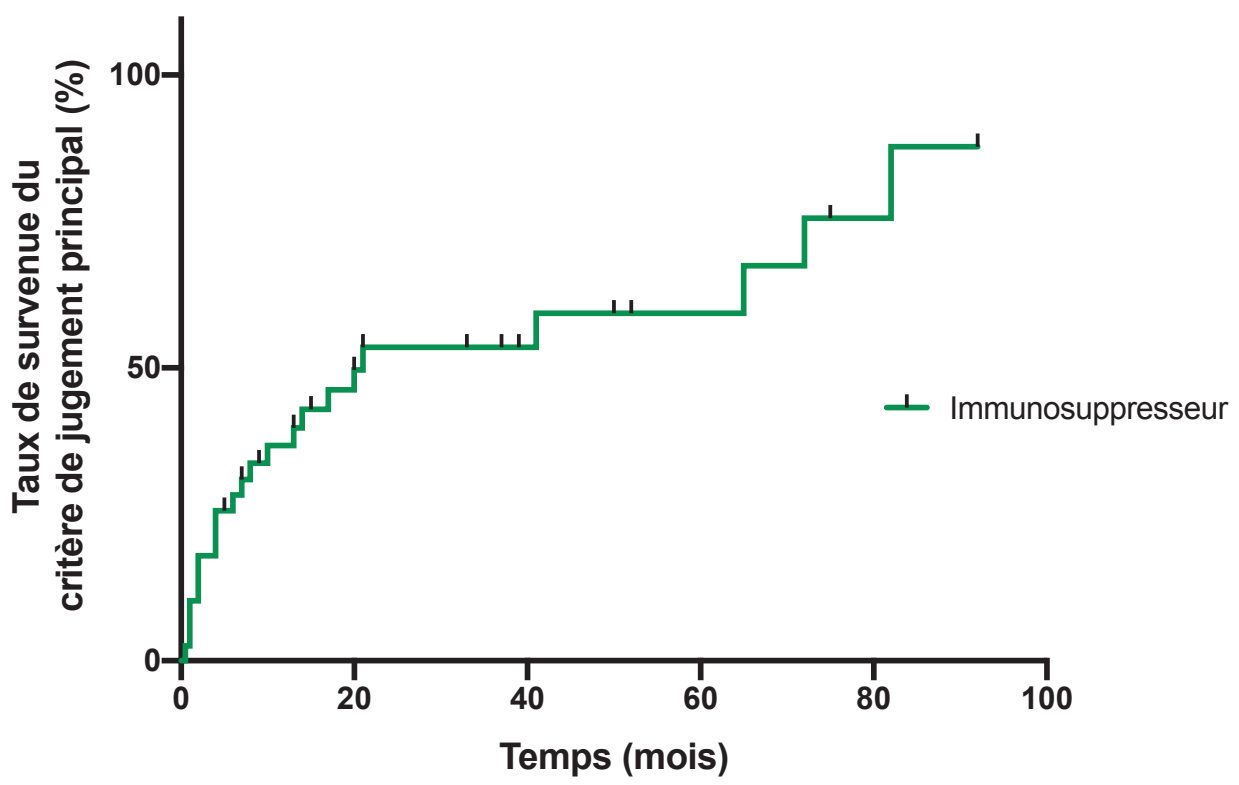

Figure 17 : Courbe de Kaplan-Meier représentant la survenue du critère de jugement principal lors du traitement par immunosuppresseurs conventionnels. Les tirets verticaux représentent les données censurées.

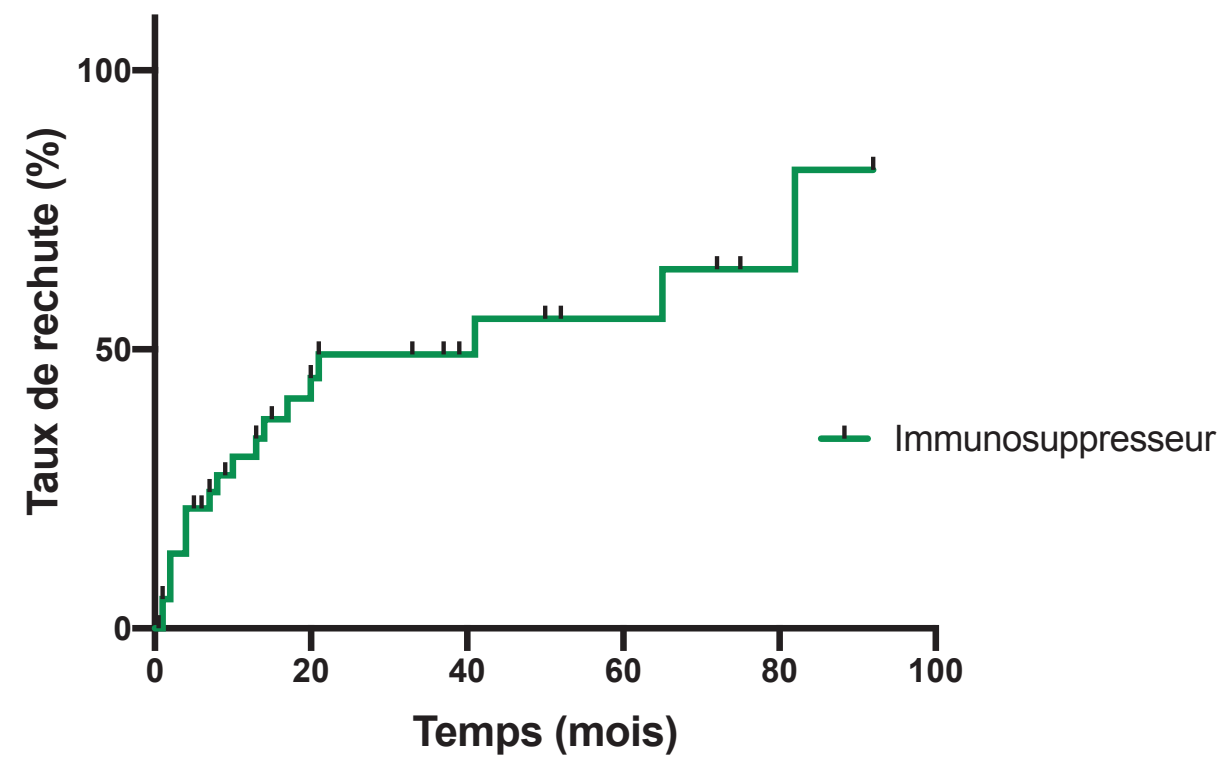

Figure 18 : Courbe de Kaplan-Meier représentant la survenue d'une rechute lors du traitement par immunosuppresseurs conventionnels. Les tirets verticaux représentent les données censurées. 
Si on s'attache à l'analyse du taux de rechute, 19 patients ont présenté une rechute avec un délai médian de rechute de 41 mois (Figure 18). La posologie médiane de corticoïdes lors de la rechute était de $10 \mathrm{mg}$ [6,5 - 21]. Une seule rechute est survenue lors de la décroissance de la posologie du traitement immunosuppresseur (mycophénolate mofétil). Lors de la rechute, les patients ont été pris en charge de la manière suivante : un changement de traitement pour l'azathioprine pour 3 patients traités par méthotrexate, un changement de traitement pour l'interféron alpha-2a pour 4 patients (deux traités par méthotrexate, un recevant du mycophénolate mofétil et le dernier bénéficiant d'azathioprine), un relais par adalimumab pour 2 patients recevant du mycophénolate mofétil (non débuté à la fin de l'étude), une reprise du mycophénolate mofétil à $2 \mathrm{~g} /$ jour (rechute survenue lors de la décroissance de la posologie), une majoration de la corticothérapie per os pour 5 patients (2 sous méthotrexate, 2 sous mycophénolate mofétil et un sous azathioprine), une injection sous-conjonctivale de corticoïdes pour 2 patients (méthotrexate et azathioprine), la mise en place d'un implant intra-vitréen de corticoïdes (méthotrexate) et la reprise de collyres de corticoïdes (méthotrexate).

\subsection{4. Évolution des paramètres ophtalmologiques sous traitement}

Après un an de traitement $(\mathrm{n}=21)$, l'acuité visuelle (LogMAR) médiane (IQR) était de $0,05[0-0,15]$ pour l'œil droit et de $0[0-0,22]$ pour l'œil gauche (Figure 19.A). Cette variation temporelle de l'acuité visuelle sous traitement était statistiquement significative pour l'œil gauche uniquement (œil droit : $p=0,13$; œil gauche : $p=0,02$ ). Au niveau de l'œil droit, après un mois de traitement (Figure 19.C), $3 \%$ des patients présentaient une amélioration de l'acuité visuelle et $97 \%$, une stabilité de celle-ci. A 6 mois de traitement $(\mathrm{n}=29), 7 \%$ des patients présentaient une amélioration de leur acuité, $86 \%$ une stabilité et $7 \%$ une dégradation. Enfin, à 12 mois $(\mathrm{n}=21)$, le taux d'amélioration s'élevait à $5 \%, 90 \%$ des patients observaient une stabilité de leur acuité visuelle et $5 \%$ une dégradation de celle-ci. Concernant l'œil gauche (Figure 19.D), $13 \%$ et $87 \%$ des patients présentaient respectivement une amélioration ou une stabilité de leur acuité visuelle à un mois de traitement. A 6 mois de traitement, $7 \%$ des cas montraient une amélioration, $86 \%$ une stabilité et $7 \%$ une dégradation de leur acuité visuelle. Enfin, à 12 mois de traitement, on observait une amélioration, une stabilité et une dégradation de l'acuité dans respectivement $11 \%, 84 \%$ et $5 \%$ des cas.

Concernant l'efficacité du traitement sur l'inflammation intraoculaire, à l'introduction du traitement immunosuppresseur, $51 \%$ des patients présentaient un état de quiescence oculaire. Ce taux s'élevait à $62 \%$ à un mois, $68 \%$ à 6 mois et $76 \%$ à 12 mois. 
19.A

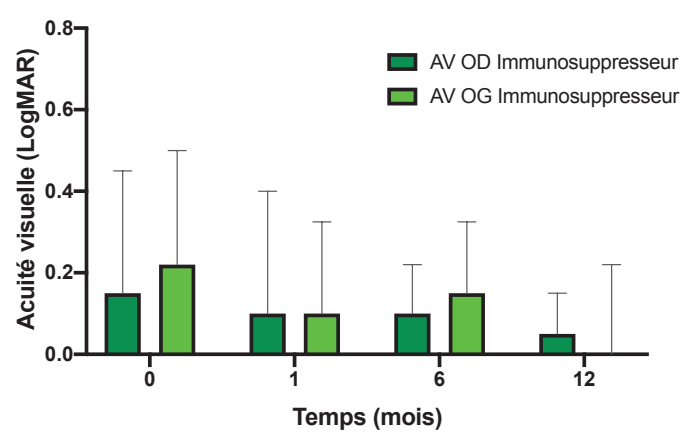

19.C

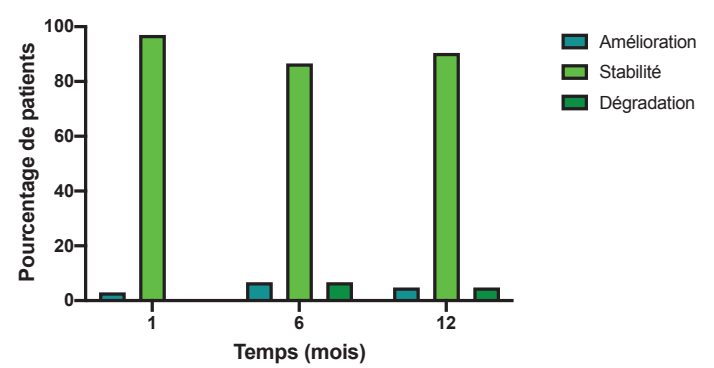

19.B

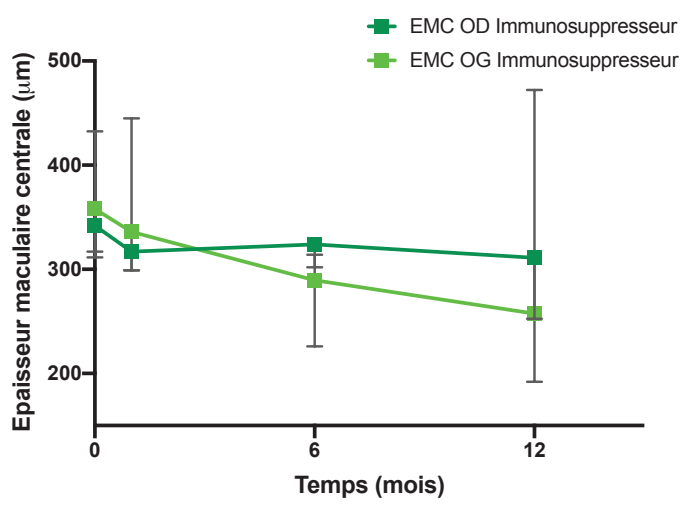

19.D

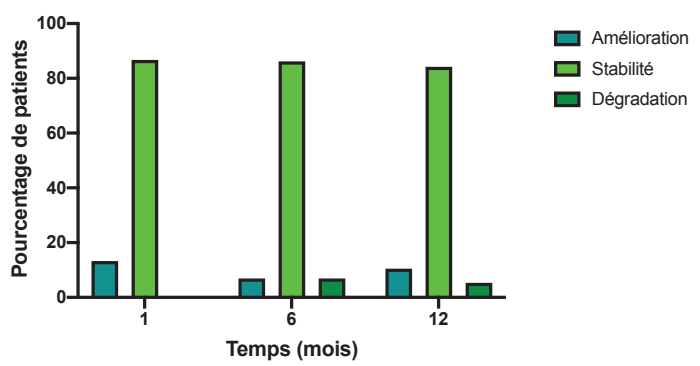

Figure 19 : Évolution de l'acuité visuelle (AV) (19.A) et de l'épaisseur maculaire centrale (EMC) (19.B) au cours des 12 premiers mois de traitement par immunosuppresseurs conventionnels. Évolution de l'acuité visuelle au cours des 12 premiers mois selon les critères d'amélioration, de stabilité ou de dégradation pour l'œil droit (19.C) et l'œil gauche (19.D). OD : œil droit. OG : œil gauche. Représentation graphique des médianes et IQR (interquartile range). 
Le dernier paramètre étudié était la variation de l'épaisseur maculaire centrale en OCT. L'épaisseur maculaire centrale médiane (IQR) était initialement de $342 \mu \mathrm{m}$ [317 - 404] pour l'œil droit et $358 \mu \mathrm{m}$ [312 - 433] pour l'œil gauche (Figure 19.B). Après 6 mois de traitement, on observait une stagnation de l'épaisseur maculaire de l'œil droit (324 $\mu \mathrm{m}$ [302 - 395]) et une normalisation de l'épaisseur pour l'œil gauche (290 $\mu \mathrm{m}$ [226 - 314]). Enfin, après un an de traitement, les chiffres restaient similaires (311 $\mu \mathrm{m}$ [253 - 417] pour l'œil droit et $258 \mu \mathrm{m}$ [192 - 472] pour l'œil gauche). Cette diminution de l'épaisseur maculaire au cours du temps n'était pas statistiquement significative ( $\mathrm{p}=0,60$ pour l'œil droit et $\mathrm{p}=0,27$ pour l'œil gauche). Une correction de l'œdème maculaire a pu être observée chez $48 \%$ des patients (11 patients). Parmi ces patients, 3 ont été considérés comme ayant présenté une rechute sur d'autres paramètres que l'œdème maculaire. Parmi les 12 patients pour lesquels le traitement n'a pas permis une amélioration de l'œdème maculaire, 11 ont nécessité une intensification thérapeutique.

Enfin, la cicatrisation des lésions de vascularite, caractère de sévérité des uvéites, a été observée chez $71 \%$ des patients (10 patients). Parmi les 4 patients n'ayant pas eu d'amélioration de la vascularite sous traitement, 2 ont été considérés comme présentant une rechute devant une amélioration insuffisante des lésions vasculaires.

\subsubsection{Caractéristiques à la fin du suivi}

Seize patients (41\%) n'ont pas présenté de rechute. La durée médiane de suivi pour ces patients était de 21 mois $[10$ - 47]. Cinq patients ont pu arrêter le traitement immunosuppresseur : 3 patients recevaient de l'azathioprine et 2 patients étaient traités par méthotrexate. A noter que parmi ces 5 patients, 3 patients ont présenté une rechute plus de 6 mois après l'arrêt du traitement.

L'acuité visuelle médiane (IQR) à la fin du suivi était conservée avec des chiffres de $0,025[0-0,25]$ pour l'œil droit et $0[0-0,15]$ pour l'œil gauche. Seul un patient présentait un œdème maculaire. Une cataracte était apparue chez 2 patients. La posologie médiane quotidienne (IQR) de prednisone à la fin du suivi était de $6 \mathrm{mg} /$ jour [4,3 - 10]. 


\subsubsection{Tolérance et effets indésirables}

Quinze patients (38\%) ont présenté au moins un effet indésirable dont 4 patients (10\%) un effet sévère ayant nécessité l'arrêt du traitement : diarrhées fébriles sous azathioprine (2 patients), pancréatite aiguë sous azathioprine et pneumocystose sous méthotrexate.

Parmi les 18 effets indésirables totaux recensés, 5 épisodes infectieux ont pu être relevés (13\%). Outre l'épisode de pneumocystose, 4 épisodes infectieux non graves, pris en charge en ambulatoire, sont survenus : infections respiratoires (un cas sous méthotrexate et un cas sous mycophénolate mofétil), verrues florides (azathioprine) et abcès dentaire (méthotrexate).

Les effets indésirables notables étaient majoritairement de type digestifs, touchant $15 \%$ des patients $(n=6)$. Ces anomalies digestives étaient majoritairement survenues sous azathioprine (5 patients). Lors de ce traitement, deux patients ont présenté des diarrhées fébriles, un patient une pancréatite et deux patients une perturbation du bilan hépatique. Un patient a présenté des nausées sous méthotrexate.

Deux patients ont développé une néoplasie au cours du suivi. Une patiente traitée par azathioprine a développé un cancer mammaire 5 ans après le début du traitement, pris en charge de manière chirurgicale. Un patient a développé un adénocarcinome pulmonaire 9 ans après le début du traitement par méthotrexate, pris en charge par lobectomie. Pour ces deux patients, la néoplasie est survenue après l'arrêt du traitement. Aucun des deux patients n'est décédé.

\subsubsection{Analyse de sous-groupes selon le type d'immunosuppresseur conventionnel reçu}

Les patients traités par azathioprine étaient plus jeunes, avec un âge médian (IQR) au début du traitement de 40 ans [29 - 51]. La répartition étiologique était hétérogène entre les différents traitements immunosuppresseurs. En effet, seuls les patients atteints d'une maladie de birdshot ont reçu du mycophénolate mofétil. Le méthotrexate était indiqué dans $50 \%$ des cas pour la prise en charge d'une uvéite sarcoïdosique et dans $43 \%$ pour une uvéite idiopathique. Les indications de l'azathioprine étaient plus disparates : $33 \%$ pour une uvéite idiopathique, $27 \%$ pour une maladie de Vogt-Koyanagi-Harada et $20 \%$ pour une maladie de Behçet. Seul l'azathioprine a été utilisé dans la prise en charge de la maladie de Behçet et la maladie de Vogt-Koyanagi-Harada (Tableau 8).

Sur le plan ophtalmologique, respectivement $50 \%$ et $47 \%$ des patients traités par mycophénolate mofétil et azathioprine présentaient une vascularite active à l'angiographie. Un œdème maculaire initial était relevé chez $79 \%$ des patients sous méthotrexate et $70 \%$ des 
patients sous mycophénolate mofétil. La durée médiane (IQR) d'évolution de l'uvéite et donc, d'exposition antérieure à la corticothérapie, était plus courte chez les patients traités par mycophénolate mofétil $(3,5$ mois $[2-11])$.

Le critère de jugement principal a été atteint chez $79 \%$ des patients traités par méthotrexate, $40 \%$ des patients sous azathioprine et $60 \%$ des patients recevant du mycophénolate mofétil $(\mathrm{p}=0,11)$. Une rechute a été observée chez $71 \%$ des patients sous méthotrexate, $60 \%$ des cas prenant du mycophénolate mofétil et $20 \%$ pour le groupe azathioprine $(\mathrm{p}=0,02)$. Enfin, un effet indésirable a été observé chez $20 \%$ des patients sous azathioprine, $7 \%$ des patients prenant du méthotrexate et aucun patient sous mycophénolate mofétil $(\mathrm{p}=0,24)$. 


\begin{tabular}{|c|c|c|c|c|}
\hline & Azathioprine & Méthotrexate & $\begin{array}{l}\text { Mycophénolate } \\
\text { mofétil }\end{array}$ & $\mathbf{p}$ \\
\hline Effectif (valeur absolue et \%) & $15(38)$ & $14(36)$ & $10(26)$ & \\
\hline Caractéristiques générales de la population & & & & \\
\hline Age median (IQR) & $40[29-51]$ & 59 [33 - 82] & $52[47-61]$ & \\
\hline Sexe (homme) & $5(33)$ & $5(36)$ & $5(50)$ & \\
\hline \multicolumn{5}{|l|}{ Etiologie de l'uvéite } \\
\hline Idiopathique & $5(33)$ & $6(43)$ & 0 & \\
\hline Maladie de birdshot & 0 & 0 & $10(100)$ & \\
\hline Maladie de Behçet & $3(20)$ & 0 & 0 & \\
\hline Choroïdite multi-focale & 0 & 0 & 0 & \\
\hline Sarcoïdose & $2(13)$ & $7(50)$ & 0 & \\
\hline Vogt-Koyanagi-Harada & $4(27)$ & 0 & 0 & \\
\hline Autres & $1(7)$ & $1(7)$ & 0 & \\
\hline \multicolumn{5}{|l|}{ Caractéristiques ophtalmologiques initiales } \\
\hline Bilatéralité & $12(80)$ & $14(100)$ & $10(100)$ & \\
\hline \multicolumn{5}{|l|}{ Localisation uvéite } \\
\hline intermédiaire & $1(7)$ & $1(7)$ & 0 & \\
\hline postérieure & $5(33)$ & 0 & $10(100)$ & \\
\hline panuvéite & $9(60)$ & $13(93)$ & 0 & \\
\hline $\begin{array}{l}\text { Acuité visuelle médiane (LogMAR) de l'œil droit } \\
\text { (OD) [IQR] }\end{array}$ & $\begin{array}{c}0,1 \\
{[0-0,40]}\end{array}$ & $\begin{array}{c}0,19 \\
{[0,11-0,48]}\end{array}$ & $\begin{array}{c}0,2 \\
{[0,10-0,55]}\end{array}$ & \\
\hline $\begin{array}{l}\text { Acuité visuelle médiane (LogMAR) de l'œil gauche } \\
\text { (OG) [IQR] }\end{array}$ & $\begin{array}{c}0,15 \\
{[0-0,45]}\end{array}$ & $\begin{array}{c}0,33 \\
{[0,10-0,70]}\end{array}$ & $\begin{array}{c}0,3 \\
{[0,09-0,33]}\end{array}$ & \\
\hline Uvéite granulomateuse & $2(13)$ & $2(14)$ & 0 & \\
\hline Vascularite active & $7(47)$ & $2(14)$ & $5(50)$ & \\
\hline OEdème maculaire cystoïde & $5(33)$ & $11(79)$ & $7(70)$ & \\
\hline $\begin{array}{l}\text { épaisseur maculaire centrale médiane ( } \mu \mathrm{m} \text { en } \\
\text { OCT) OD [IQR] }\end{array}$ & $\begin{array}{c}316 \\
{[300-412]}\end{array}$ & $\begin{array}{c}396 \\
{[339-415]}\end{array}$ & $\begin{array}{c}330 \\
{[323-342]}\end{array}$ & \\
\hline $\begin{array}{l}\text { épaisseur maculaire centrale médiane ( } \mu \mathrm{m} \text { en } \\
\text { OCT) OG [IQR] }\end{array}$ & $\begin{array}{c}328 \\
{[303-352]}\end{array}$ & $\begin{array}{c}397 \\
{[323-460]}\end{array}$ & $\begin{array}{c}336 \\
{[301-603]}\end{array}$ & \\
\hline \multicolumn{5}{|l|}{ Prise en charge thérapeutique } \\
\hline Durée médiane (mois) d'évolution de l'uvéite [IQR] & $\begin{array}{c}13 \\
{[5-31]}\end{array}$ & $\begin{array}{c}14 \\
{[6-29]}\end{array}$ & $\begin{array}{c}3,5 \\
{[2-11]}\end{array}$ & \\
\hline $\begin{array}{l}\text { Durée médiane (mois) d'exposition antérieure au } \\
\text { traitement à la corticothérapie [IQR] }\end{array}$ & $\begin{array}{c}12 \\
{[4-31]}\end{array}$ & $\begin{array}{c}9,5 \\
{[3-27]}\end{array}$ & $\begin{array}{c}3 \\
{[0-9]}\end{array}$ & \\
\hline \multicolumn{5}{|l|}{ Critère de jugement principal } \\
\hline $\begin{array}{l}\text { Nombre de patients ayant atteint le critère de } \\
\text { jugement principal }\end{array}$ & $6(40)$ & $11(79)$ & $6(60)$ & 0,11 \\
\hline rechute & $3(20)$ & $10(71)$ & $6(60)$ & 0,02 \\
\hline effet indésirable sévère & $3(20)$ & $1(7)$ & 0 & 0,24 \\
\hline
\end{tabular}

Tableau 8 : Analyse des caractéristiques ophtalmologiques, de la prise en charge thérapeutique et du critère de jugement principal selon l'immunosuppresseur reçu. Les données qualitatives sont exprimées en valeur absolue et en pourcentages. Les données quantitatives sont exprimées en médiane et IQR. IQR : interquartile range. OD : œil droit. OG : œil gauche. OCT : tomographie par cohérence optique. 


\subsection{Comparaison des deux stratégies thérapeutiques}

3.4.1. Caractéristiques générales et ophtalmologiques lors de l'introduction du $\underline{\text { traitement }}$

A l'introduction du traitement immunosuppresseur conventionnel ou de la biothérapie, il n'y avait pas de différence significative entre les deux groupes, tant au niveau des caractéristiques générales de la population que de l'étiologie de l'uvéite (Tableau 9). La répartition étiologique était homogène concernant les deux diagnostics principaux. Cependant, la maladie de Behçet était plus représentée dans le groupe biothérapie de façon non significative (19\% versus $8 \%, p=0,18)$, de même, la sarcoïdose était plus représentée dans le groupe immunosuppresseur conventionnel (23\% versus $8 \%, p=0,12)$. Les choroïdites multi-focales, quant à elles, étaient uniquement retrouvées dans le groupe biothérapie, de même que les maladies de Vogt-Koyanagi-Harada étaient traitées uniquement par immunosuppresseurs conventionnels.

La médiane de l'acuité visuelle (LogMAR) à l'introduction du traitement n'était pas statistiquement différente entre les deux groupes. De plus, il n'y avait pas de différence significative entre les deux groupes concernant la sévérité de l'uvéite (présence d'une vascularite active et/ou d'un œdème maculaire cystoïde) lors de l'introduction du traitement.

\subsubsection{Prise en charge thérapeutique}

La durée d'évolution de l'uvéite était significativement plus longue dans le groupe biothérapie (16 mois versus 11 mois ; $p=0,03$ ), et de même, les patients étaient exposés depuis plus longtemps à une corticothérapie (13 mois versus 8 mois ; $p=0,03)$. Enfin, les patients du groupe biothérapie avaient plus fréquemment rechuté avant l'introduction de ce traitement, comparés aux patients traités par immunosuppresseurs conventionnels $(p=0,01)$. En revanche, il n'y avait pas de différence entre les deux groupes en termes de niveau de cortico-dépendance initial $(\mathrm{p}=0,1)$ lors de l'introduction du traitement (Tableau 10).

A 6 mois de traitement, la posologie médiane journalière de corticoïdes était de $11 \mathrm{mg}$ [7 - 17] chez les patients traités par biothérapies versus $10 \mathrm{mg}$ [10 - 16] chez les patients recevant un immunosuppresseur conventionnel. 


\begin{tabular}{|c|c|c|c|}
\hline & Biothérapie & $\begin{array}{c}\text { Immuno- } \\
\text {-suppresseur }\end{array}$ & p \\
\hline $\begin{array}{l}\text { Effectif (valeur absolue et \%) } \\
\text { Caractéristiques qénérales }\end{array}$ & $36(48)$ & $39(52)$ & \\
\hline Age median $[\mathrm{IQR}]$ & $43[32-62]$ & $49[34-61]$ & 0,48 \\
\hline Sexe (homme) & $22(61)$ & $15(38)$ & 0,08 \\
\hline Antécédents & & & \\
\hline HTA & $6(17)$ & $8(21)$ & 0,9 \\
\hline diabète & 0 & $3(8)$ & 0,24 \\
\hline $\begin{array}{l}\text { Etiologie de l'uvéite } \\
\text { Idionathique }\end{array}$ & & & \\
\hline Idiopathique & $12(33)$ & $11(28)$ & 0,80 \\
\hline Maladie de birdshot & $9(25)$ & $10(26)$ & $>0,99$ \\
\hline Maladie de Behçet & $7(20)$ & $3(8)$ & 0,18 \\
\hline Sarcoïdose & $3(8)$ & $9(23)$ & 0,12 \\
\hline Choroïdite multifocale & $3(8)$ & 0 & 0,1 \\
\hline Vogt-Koyanagi-Harada & 0 & $4(10)$ & 0,11 \\
\hline Autres & $2(6)$ & $2(5)$ & \\
\hline lupus érythémateux systémique & 0 & 1 & \\
\hline ophtalmie sympathique & 0 & 1 & \\
\hline rhumatisme psoriasique & 1 & 0 & \\
\hline maladie de Crohn & 1 & 0 & \\
\hline Caractéristiques ophtalmologiques à & & & \\
\hline l'introduction du traitement & 35 & & $>0.99$ \\
\hline Localisation uvéite & $30(91)$ & $36(92)$ & \\
\hline intermédiaire & $2(6)$ & $2(5)$ & \\
\hline postérieure & $18(50)$ & $15(39)$ & \\
\hline panuvéite & $16(44)$ & $22(56)$ & 0,57 \\
\hline $\begin{array}{l}\text { Acuité visuelle médiane (LogMAR) de l'œil } \\
\text { droit (OD) [IQR] }\end{array}$ & $0,35[0-0,5]$ & $0,15[0,1-0,45]$ & 0,65 \\
\hline $\begin{array}{l}\text { Acuité visuelle médiane (LogMAR) de l'œil } \\
\text { gauche (OG) [IQR] }\end{array}$ & $0,15[0,025-0,50]$ & $0,22[0,10-0,50]$ & 0,79 \\
\hline Tyndall protéique (SUN) & $\mathrm{n}=35$ & $\mathrm{n}=37$ & \\
\hline 0 & $18(51)$ & $18(49)$ & 0,95 \\
\hline $0,5+$ & $7(20)$ & $9(24)$ & \\
\hline $1+$ & $7(20)$ & $6(16)$ & \\
\hline $2+$ & $2(6)$ & $3(8)$ & \\
\hline $3+$ & $1(3)$ & 0 & \\
\hline $4+$ & 0 & $1(3)$ & \\
\hline Flou vitréen (Nussenblatt) & $\mathrm{n}=35$ & $\mathrm{n}=37$ & \\
\hline 0 & $12(34)$ & $15(41)$ & 0,73 \\
\hline $0,5+$ & $6(17)$ & $9(24)$ & \\
\hline $1+$ & $14(40)$ & $12(32)$ & \\
\hline $2+$ & $2(6)$ & $1(3)$ & \\
\hline $3+$ & $1(3)$ & 0 & \\
\hline Uvéite granulomateuse & $2(6)$ & $4(10)$ & 0,67 \\
\hline Vascularite active & $13(36)$ & $14(36)$ & 0,85 \\
\hline OEdème maculaire cystoïde & $20(56)$ & 23 (59) & 0,85 \\
\hline $\begin{array}{l}\text { médiane de l'épaisseur maculaire } \\
\text { centrale (um en OCT) OD [IQR] }\end{array}$ & $433[323-555]$ & $342[317-404]$ & 0,07 \\
\hline $\begin{array}{l}\text { médiane de l'épaisseur maculaire } \\
\text { centrale }(\mu \mathrm{m} \text { en OCT) OG [IQR] }\end{array}$ & $355[313-447]$ & 358 [312 - 433] & 0,92 \\
\hline
\end{tabular}

Tableau 9 : Caractéristiques générales et ophtalmologiques lors de l'introduction du traitement d'étude. Les données qualitatives sont exprimées en valeur absolue et en pourcentages. Les données quantitatives sont exprimées en médiane et IQR. IQR : interquartile range. OD : œil droit. OG : œil gauche. SUN : Standardization of uveitis nomenclature. OCT : tomographie par cohérence optique. 


\begin{tabular}{|c|c|c|c|}
\hline & Biothérapie & $\begin{array}{l}\text { Immuno- } \\
\text { suppresseur }\end{array}$ & p \\
\hline Durée médiane (mois) de suivi [IQR] & $9,5[4-22]$ & $13[4-37]$ & 0,41 \\
\hline $\begin{array}{l}\text { Durée médiane (mois) d'évolution de l'uvéite } \\
\text { [IQR] }\end{array}$ & $16[9-66]$ & $11[4-27,5]$ & 0,03 \\
\hline $\begin{array}{l}\text { Durée médiane (mois) d'exposition } \\
\text { antérieure à la corticothérapie [IQR] }\end{array}$ & $13[7-62]$ & $8[3-22,5]$ & 0,03 \\
\hline Seuil de cortico-dépendance $\geq 20$ mg/jour & $18(50)$ & $12(31)$ & 0,1 \\
\hline Seuil de cortico-dépendance $\geq 35 \mathrm{mg} / \mathrm{jour}$ & $7(19)$ & $6(15)$ & 0,76 \\
\hline $\begin{array}{l}\text { Nombre médian de rechutes antérieures } \\
\text { [IQR] }\end{array}$ & $1,5[0-3]$ & $0[0-1]$ & 0,01 \\
\hline \multicolumn{4}{|l|}{ Caractéristiques du traitement en cours } \\
\hline \multicolumn{4}{|l|}{ Traitement par biothérapie } \\
\hline adalimumab & $19(53)$ & 0 & \\
\hline infliximab & $15(41)$ & 0 & \\
\hline tocilizumab & $2(6)$ & 0 & \\
\hline \multicolumn{4}{|l|}{$\begin{array}{l}\text { Traitement par immunosuppresseur } \\
\text { conventionnel }\end{array}$} \\
\hline azathioprine & 0 & $15(38)$ & \\
\hline méthotrexate & 0 & $14(36)$ & \\
\hline mycophénolate mofétil & 0 & $10(26)$ & \\
\hline \multicolumn{4}{|l|}{$\begin{array}{l}\text { Caractéristiques de la prise en charge } \\
\text { thérapeutique antérieure }\end{array}$} \\
\hline \multicolumn{4}{|l|}{ Nombre de ligne thérapeutique antérieure } \\
\hline aucun & $11(31)$ & $39(100)$ & $10^{-11}$ \\
\hline une ligne & $15(41)$ & 0 & \\
\hline deux lignes & $10(28)$ & 0 & \\
\hline \multicolumn{4}{|l|}{$\begin{array}{l}\text { Traitement antérieur par immunosuppresseur } \\
\text { conventionnel }\end{array}$} \\
\hline méthotrexate & $8(22)$ & 0 & \\
\hline azathioprine & $11(31)$ & 0 & \\
\hline mycophénolate mofétil & $9(25)$ & 0 & \\
\hline autre & $1(3)$ & 0 & \\
\hline Traitement antérieur par interféron alpha-2a & $7(19)$ & 0 & \\
\hline Sevrage en corticoïdes & $8(22)$ & $5(13)$ & 0,37 \\
\hline
\end{tabular}

Tableau 10 : Caractéristiques de la prise en charge thérapeutique. Les données qualitatives sont exprimées en valeur absolue et en pourcentages. Les données quantitatives sont exprimées en médiane et IQR. IQR : interquartile range. 
A 12 mois de traitement, alors que la posologie stagnait dans le groupe immunosuppresseur conventionnel, elle était de $7 \mathrm{mg} /$ jour dans le groupe biothérapie [5 - 10] (Figure 20). Cependant, il n'a pas été observé de différence significative en termes d'épargne cortisonique entre les deux groupes $(\mathrm{p}=0,26)$. A 12 mois de traitement, $83 \%$ des patients traités par biothérapies et $78 \%$ des patients recevant un traitement immunosuppresseur conventionnel bénéficiaient d'une posologie de corticoïdes $\leq 10 \mathrm{mg} /$ jour $(\mathrm{p}>0,99)$. Ces taux s'élevaient à $44 \%$ des patients sous biothérapies et $22 \%$ des patients sous immunosuppresseurs conventionnels si on s'intéressait aux patients recevant une dose $\leq 5 \mathrm{mg} /$ jour $(\mathrm{p}=0,28)$. Vingtdeux pour cent des patients sous biothérapies ont été sevré en corticoïdes contre $13 \%$ des patients traités par immunosuppresseurs conventionnels $(\mathrm{p}=0,37)$.

Nous avons étudié l'impact individuel en termes d'épargne cortisonique des deux stratégies thérapeutiques. Parmi les patients ayant présenté une rechute sous biothérapies $(\mathrm{n}=15), 10$ patients avaient déjà rechuté antérieurement lors du traitement par immunosuppresseurs conventionnels. La posologie médiane de corticoïdes lors de la rechute sous biothérapies était de $11 \mathrm{mg}$ [6 - 19]. En revanche, chez un même patient, la posologie de corticoïdes lors de la rechute sous immunosuppresseurs conventionnels était de $33 \mathrm{mg}$ [16-40]. Cette différence de posologie lors de la rechute était statistiquement significative $(p=0,001)$. 


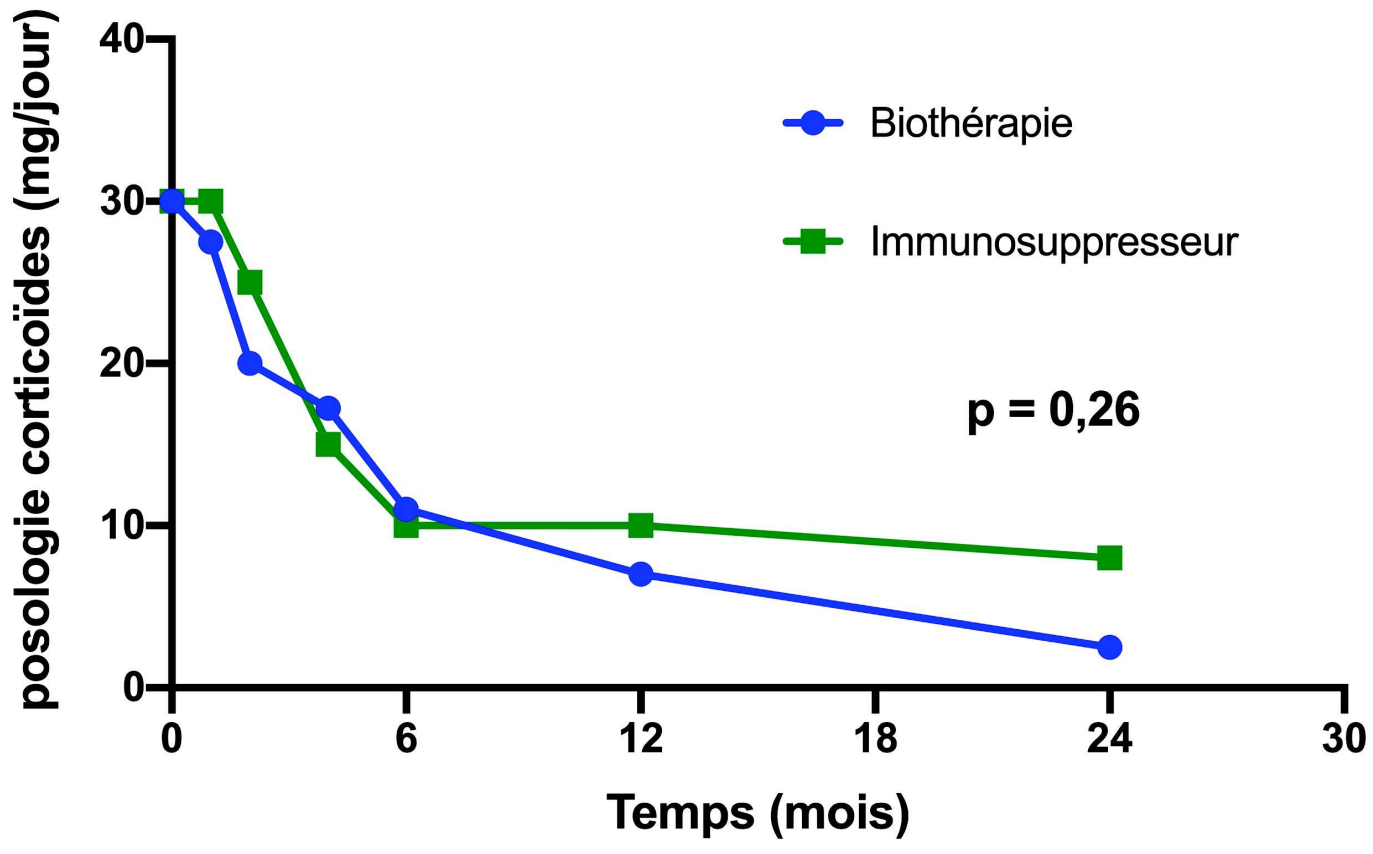

Figure 20 : Évolution de la posologie médiane quotidienne de prednisone (mg/jour) au cours du temps, en fonction du traitement étudié.

\begin{tabular}{|c|c|c|c|}
\hline & Biothérapie & $\begin{array}{l}\text { Immuno- } \\
\text { suppresseur }\end{array}$ & $\mathbf{p}$ \\
\hline \multicolumn{4}{|l|}{ Critère de jugement principal } \\
\hline $\begin{array}{l}\text { Nombre de patients ayant atteint le critère de jugement } \\
\text { principal }\end{array}$ & $20(56)$ & $23(59)$ & 0,95 \\
\hline rechute & $15(41)$ & $19(49)$ & 0,7 \\
\hline effet indésirable sévère & $5(14)$ & $4(10)$ & 0,73 \\
\hline Nombre de perdus de vue & $1(3)$ & $2(5)$ & $>0,99$ \\
\hline Rechute lors de la décroissance du traitement & $2(6)$ & $1(3)$ & 0,6 \\
\hline
\end{tabular}

Tableau 11 : Survenue du critère de jugement principal. Les données qualitatives sont exprimées en valeur absolue et en pourcentages. 


\subsubsection{Analyse du critère de jugement principal}

Vingt patients dans le groupe biothérapie (56\%) et 23 patients dans le groupe immunosuppresseur conventionnel (59\%) ont atteint le critère de jugement principal $(\mathrm{p}=0,95)$ (Tableau 11). Quinze patients (41\%) dans le groupe biothérapie et 19 patients (49\%) dans le groupe immunosuppresseur conventionnel ont présenté une rechute $(p=0,7)$. Cinq patients (14\%) dans le groupe biothérapie et 4 patients $(10 \%)$ dans le groupe immunosuppresseur conventionnel ont présenté un effet indésirable grave ayant nécessité d'interrompre le traitement $(p=0,73)$. Ainsi, il n'y avait pas de différence entre les deux groupes en termes de taux de rechute ou d'effets indésirables graves.

Le délai médian de survenue du critère de jugement principal était plus long dans le groupe immunosuppresseur conventionnel (21 mois versus 16 mois), sans que ce résultat soit statistiquement significatif $(p=0,47)$ (Figure 21.A). La posologie médiane de corticoïdes lors de la survenue du critère de jugement principal n'était pas différente entre les deux groupes : 9,5 $\mathrm{mg} /$ jour pour les patients traités par biothérapies versus $10 \mathrm{mg} / \mathrm{jour}$ chez les patients recevant des immunosuppresseurs conventionnels $(\mathrm{p}=0,43)$. Un mois après l'initiation $\mathrm{du}$ traitement, seuls $2,8 \%$ des patients du groupe biothérapie avaient atteint le critère de jugement principal contre 10,3\% des patients sous immunosuppresseurs conventionnels. Cet avantage s'effaçait rapidement puisque ce taux atteignait respectivement $29,6 \%$ et $28,3 \%$ des patients à 6 mois de traitement. A un an de traitement, 39,6\% des patients du groupe biothérapie avaient atteints le critère de jugement principal versus $36,7 \%$ des patients du groupe immunosuppresseur conventionnel. Le délai médian de rechute était de 29 mois pour les patients sous biothérapies et de 41 mois chez les patients traités par immunosuppresseurs conventionnels. Ce délai était plus long, dans les deux groupes, que le délai de survenue du critère de jugement principal, sans différence significative $(p=0,77)$ (Figure 21.B). L'analyse de sous-groupe portant sur les patients traités en première intention par biothérapies $(n=11)$, n'ayant donc jamais reçu d'immunosuppresseur conventionnel, comparés aux patients traités en première intention par du méthotrexate ou de l'azathioprine ou du mycophénolate moféil $(\mathrm{n}=39)$, retrouvait un délai médian de survenue du critère de jugement principal plus long (19,5 mois), mais restant non significatif comparé au groupe traité par immunosuppresseurs conventionnels (19,5 mois versus 21 mois, $p=0,67)$ (Figure 21.C). 
21.A

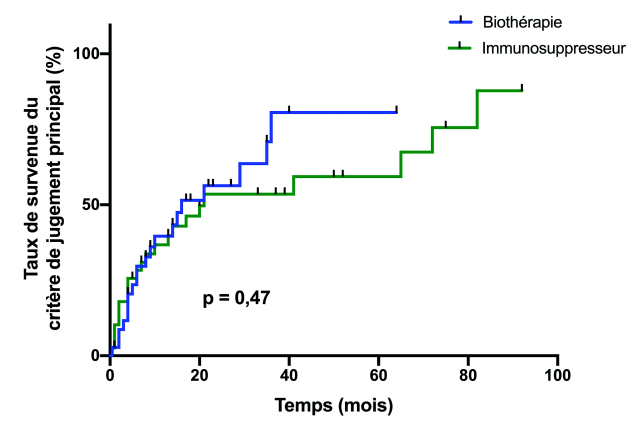

21.C

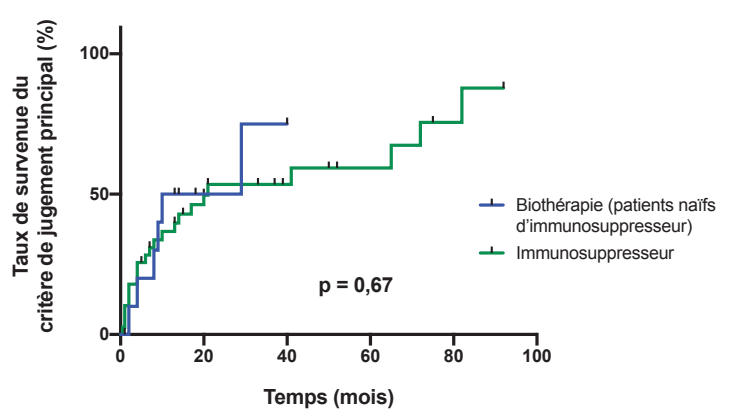

21.B

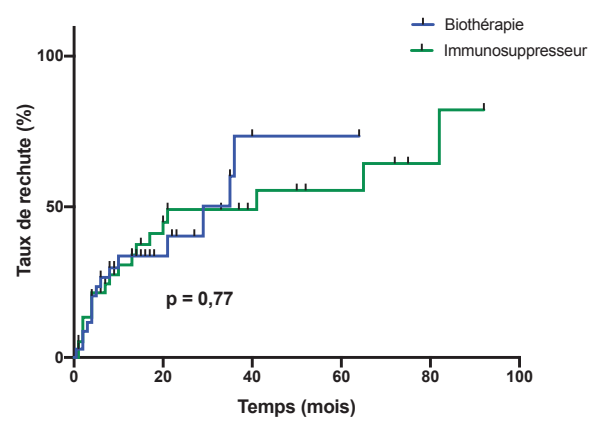

21.D

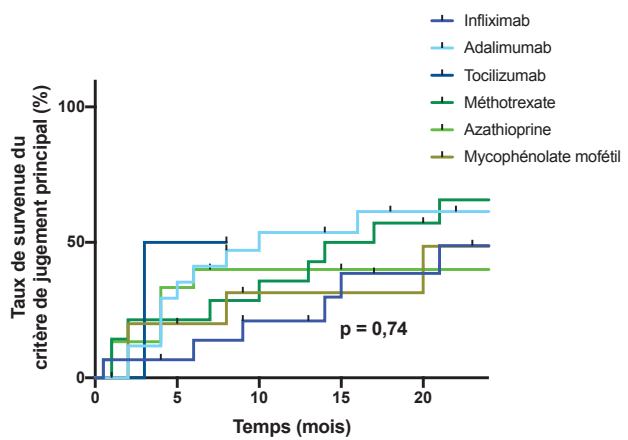

Figure 21 : Courbe de Kaplan-Meier représentant la survenue du critère de jugement principal ou d'une rechute : survenue du critère de jugement principal pour la population entière (21.A), survenue d'une rechute pour la population entière (21.B), survenue du critère de jugement principal chez les patients traités en première intention par immunosuppresseur conventionnel ou biothérapie (i.e. naïfs de traitement immunosuppresseur conventionnel antérieur) (21.C), survenue du critère de jugement principal selon le sous-groupe de traitement reçu (pour des raisons de lisibilités, données exprimées sur 24 mois) (21.D). Les tirets verticaux représentent les données censurées. 
L'analyse de sous-groupes selon le type de biothérapie et d'immunosuppresseur conventionnel reçu n'a pas montré de différence significative entre les groupes $(\mathrm{p}=0,74)$ (Figure 21.D). Le délai médian de rechute était de 5,5 mois sous tocilizumab (résultat à nuancer devant le faible effectif), de 10 mois sous adalimumab, de 15,5 mois sous méthotrexate, de 29 mois sous infliximab, de 41 mois sous mycophénolate mofétil et non calculable pour l'azathioprine.

La principale manifestation ophtalmologique signant le diagnostic de rechute dans les deux groupes était la majoration ou l'apparition d'un œdème maculaire sous traitement $(60 \%$ dans le groupe biothérapie et $53 \%$ dans le groupe immunosuppresseur ; $p=0,48$ ) (Tableau 12). Le paramètre ophtalmologique le moins fréquemment impliqué dans la rechute était l'apparition d'une inflammation au niveau du segment antérieur, pour les deux groupes. Il n'y avait pas de différence significative entre les deux groupes en termes de paramètres ophtalmologiques attestant de la rechute. De même, le délai de survenue des différents paramètres ophtalmologiques révélant la rechute n'était pas différent entre les deux groupes (Figure 22). La survenue d'une inflammation intraoculaire, tant au niveau du segment antérieur que du vitré, constituait le paramètre apparu de façon la plus précoce.

\begin{tabular}{|c|c|c|c|}
\hline $\begin{array}{c}\text { Expression ophtalmologique de la } \\
\text { rechute }\end{array}$ & Biothérapie & Immunosuppresseur & $\mathbf{p}$ \\
\hline $\begin{array}{c}\text { Apparition inflammation segment } \\
\text { antérieur }\end{array}$ & $2(13)$ & $3(16)$ & $>0,99$ \\
Apparition inflammation intra- \\
vitréenne & $4(27)$ & $5(26)$ & $>0,99$ \\
$\begin{array}{c}\text { Baisse d'acuité visuelle significative } \\
\text { Majoration/apparition d'un œdème } \\
\text { maculaire cystoïde }\end{array}$ & $6(40)$ & $6(32)$ & 0,48 \\
Absence correction/apparition d'une \\
vascularite
\end{tabular}

Tableau 12 : Paramètres ophtalmologiques signant la rechute selon le traitement étudié. Les données qualitatives sont exprimées en valeur absolue et en pourcentages. 
22.A

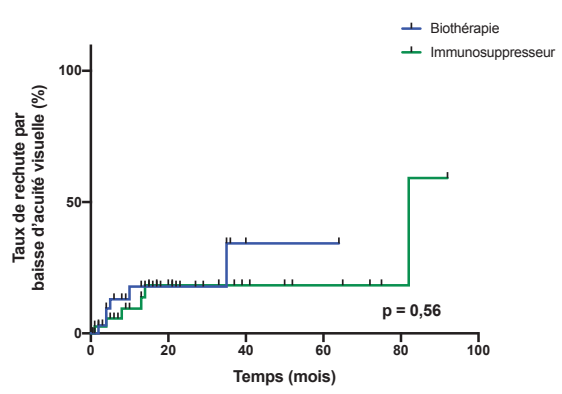

22.D

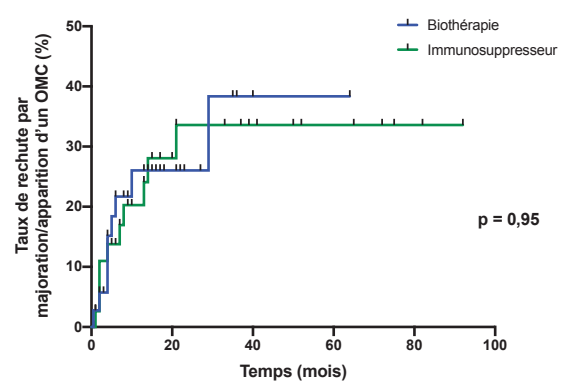

22.C

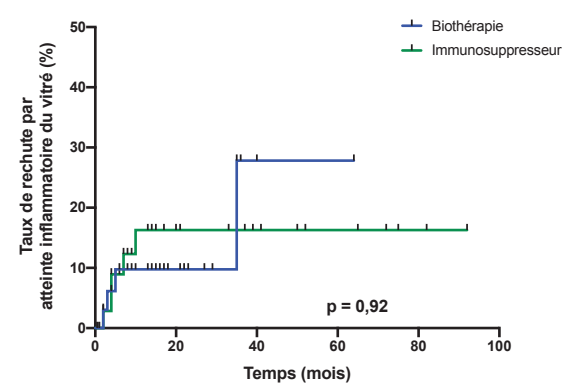

22.E

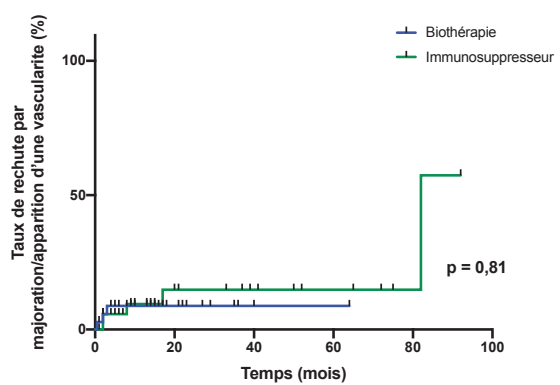

Figure 22 : Courbe de Kaplan-Meier représentant les différents paramètres ophtalmologiques signant la rechute : baisse d'acuité visuelle (22.A), inflammation du segment antérieur (22.B), inflammation vitréenne (22.C), majoration/apparition d'un œdème maculaire cystoïde (OMC) (22.D), majoration/apparition d'une vascularite (22.E). Les tirets verticaux représentent les données censurées. Pour plus de lisibilité, variation de l'échelle en ordonnées. 
La gestion de la rechute était similaire dans les deux groupes. Sept patients (60\%) dans le groupe biothérapie et 9 patients $(47 \%)$ du groupe immunosuppresseur conventionnel ont bénéficié d'un changement de traitement suite à la survenue de la rechute $(p=0,47)$. Une majoration de la corticothérapie a été observée chez uniquement 2 patients $(13 \%)$ sous biothérapies contre 9 patients $(47 \%)$ dans le groupe immunosuppresseur conventionnel $(\mathrm{p}=0,06)$.

\subsection{4. Évolution des paramètres ophtalmologiques sous traitement}

L'étude de la variation de l'acuité visuelle et de l'épaisseur maculaire centrale a montré une évolution favorable de ces paramètres ophtalmologiques sous traitement, sans différence significative entre les deux groupes (Figures 23 et 24). De plus, il n'y avait pas de différence significative entre les deux groupes concernant la résolution de l'œdème maculaire ou d'une vascularite sous traitement $(p>0,99)$.

Afin de permettre la comparaison, l'étude des critères d'amélioration et de stabilité de l'acuité visuelle a été réalisée sur l'œil le plus sévère. A chaque période d'étude, nous avons pu observer un nombre de patients présentant une amélioration de l'acuité visuelle plus important dans le groupe traité par biothérapies, mais cette différence n'était pas statistiquement significative (Tableau 13).

L'état de quiescence oculaire inflammatoire était plus fréquemment obtenu chez les patients sous biothérapies (Tableau 14), et ce résultat était statistiquement significatif à 6 mois de traitement $(\mathrm{p}=0,046)$, mais ne persistait pas à 12 mois $(\mathrm{p}=0,73)$.

Ainsi, il n'a pas été mis en évidence de différence en termes d'efficacité thérapeutique sur l'amélioration de l'acuité visuelle et la variation de l'épaisseur maculaire centrale entre les deux groupes. En revanche, l'inflammation intraoculaire était contrôlée de manière plus précoce dans le groupe traité par biothérapies, de manière significative $(\mathrm{p}=0,046)$. 
$\square$ AV OD Biothérapie

$\square$ AV OG Biothérapie

$\square$ AV OD Immunosuppresseur

$\square$ AV OG Immunosuppresseur

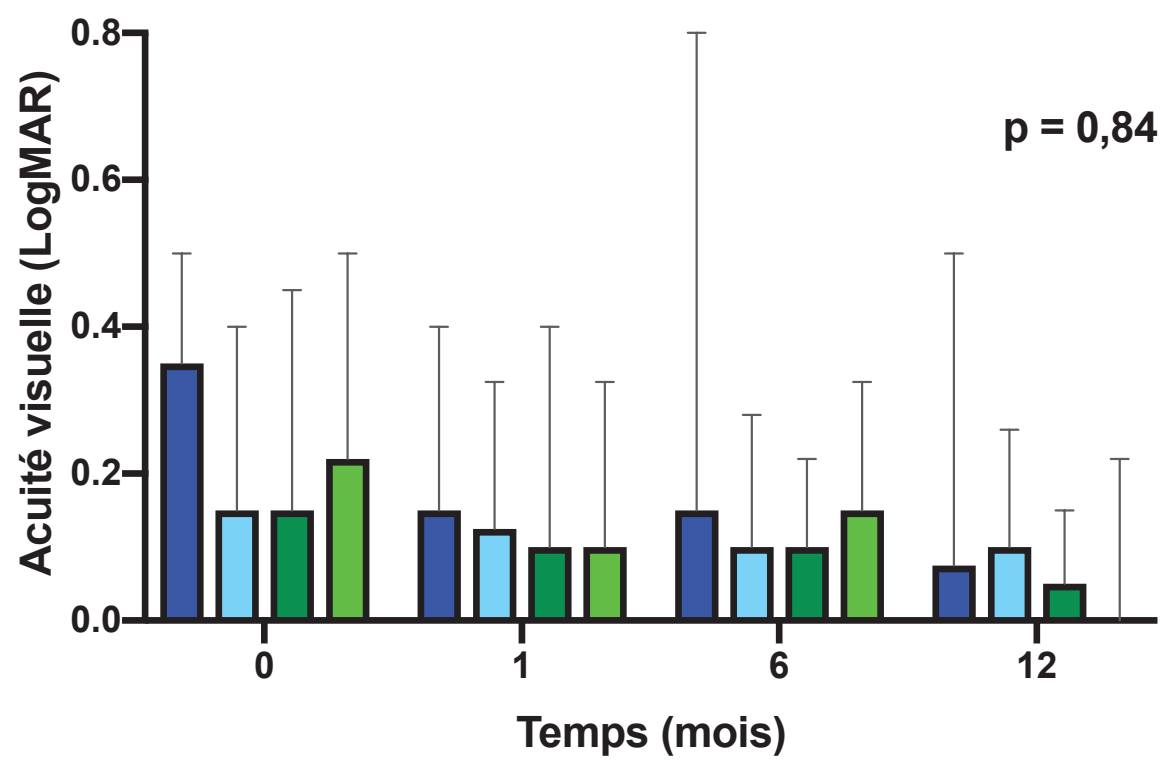

Figure 23 : Évolution de l'acuité visuelle (AV) au cours des 12 premiers mois de suivi, en fonction du traitement d'étude. OD : œil droit. OG : œil gauche.

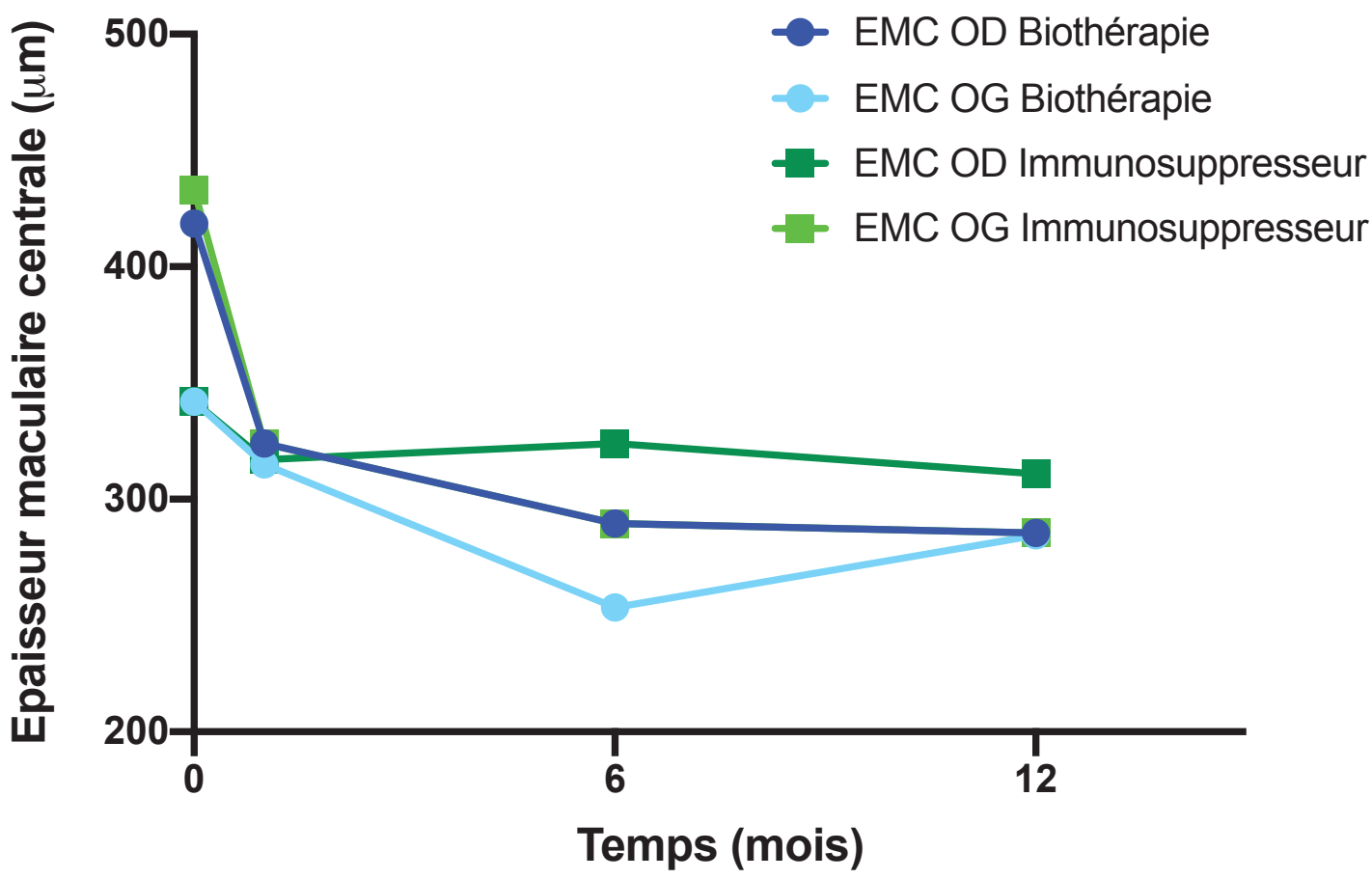

Figure 24 : Évolution de l'épaisseur maculaire centrale (EMC) au cours des 12 premiers mois de suivi, en fonction du traitement d'étude. OD : œil droit. OG : œil gauche. 


\begin{tabular}{|c|c|c|c|}
\hline & Biothérapie & Immunosuppresseur & $\mathbf{p}$ \\
\hline $\begin{array}{c}\text { Amélioration acuité visuelle (AV) à } \\
\text { un mois (M1) }\end{array}$ & $7(21)$ & $5(16)$ & 0,75 \\
Stabilité AV à M1 & $26(79)$ & $26(84)$ & 0,75 \\
Amélioration AV à M6 & $5(15)$ & $3(10)$ & \\
Stabilité AV à M6 & $24(73)$ & $24(83)$ & 0,74 \\
Dégradation AV à M6 & $4(12)$ & $2(7)$ & \\
Amélioration AV à M12 & $5(31)$ & $2(12)$ & 0,37 \\
Stabilité AV à M12 & $10(63)$ & $14(82)$ & \\
Dégradation AV à M12 & $1(6)$ & $1(6)$ & \\
\hline
\end{tabular}

Tableau 13 : Évolution de l'acuité visuelle (AV) selon les critères d'amélioration et de stabilité jusqu'à 12 mois (M) de traitement. Les données qualitatives sont exprimées en valeur absolue et en pourcentages.

\begin{tabular}{|c|c|c|c|}
\hline & Biothérapie & Immunosuppresseur & $\mathbf{p}$ \\
\hline $\begin{array}{c}\text { Etat de quiescence à l'initiation du } \\
\text { traitement }\end{array}$ & $15(41)$ & $20(51)$ & 0,49 \\
Etat de quiescence à un mois (M1) & $25(76)$ & $24(62)$ & 0,22 \\
Etat de quiescence à M6 & $23(92)$ & $20(68)$ & 0,046 \\
Etat de quiescence à M12 & $12(71)$ & $16(76)$ & 0,73 \\
\hline
\end{tabular}

Tableau 14 : Évolution de l'état de quiescence oculaire jusqu'à 12 mois (M) de traitement. Les données qualitatives sont exprimées en valeur absolue et en pourcentages. 


\subsubsection{Caractéristiques à la fin du suivi}

A la fin du suivi, 16 patients dans chaque groupe n'avaient pas présenté le critère de jugement principal. Il n'y avait pas de différence entre les deux groupes concernant les éléments de sévérité de l'uvéite (œdème maculaire, vascularite) ou les complications de l'uvéite telle l'apparition d'une cataracte. De plus, le pronostic visuel, chez ces patients n'ayant pas présenté de rechute et donc répondeurs au traitement, était similaire entre les deux groupes avec une acuité visuelle préservée (Tableau 15).

Cinq patients sous immunosuppresseurs conventionnels et 6 patients traités par biothérapies ont pu arrêter leur traitement $(\mathrm{p}>0,99)$. L'épargne cortisonique était satisfaisante dans les deux groupes, puisque la posologie médiane quotidienne de corticoïdes était $<10 \mathrm{mg}$ /jour $(5 \mathrm{mg} /$ jour pour les patients recevant des biothérapies et $6 \mathrm{mg} /$ jour pour les patients sous immunosuppresseurs conventionnels).

\begin{tabular}{|l|c|c|c|}
\hline & Biothérapie & $\begin{array}{c}\text { Immuno- } \\
\text { suppresseur }\end{array}$ & $\mathbf{p}$ \\
\hline Nombre de patients & $16(44)$ & $16(41)$ & \\
Durée médiane [IQR] de suivi (mois) & 16 & 21 & 0,17 \\
Caractéristiques ophtalmologiques à la fin du suivi & {$[8-26]$} & {$[10-47]$} & \\
Acuité visuelle (LogMAR) médiane de l'œil droit [IQR] & 0,13 & 0,025 & 0,4 \\
& {$[0-0,28]$} & {$[0-0,25]$} & \\
Acuité visuelle (LogMAR) médiane de l'œil gauche [IQR] & 0 & 0 & 0,78 \\
& {$[0-0,10]$} & {$[0-0,15]$} & \\
Vascularite active & $2(13)$ & $1(6)$ & 0,6 \\
Cataracte & $2(13)$ & $2(13)$ & $>0,99$ \\
CEdème maculaire & $1(6)$ & $1(7)$ & $>0,99$ \\
Posologie médiane [IQR] de corticoïdes à la fin du & {$[0-9,8]$} & {$[4,3-10]$} & 0,28 \\
suivi (mg/jour) & $6(38)$ & $5(31)$ & $>0,99$ \\
Arrêt du traitement & $1(3)$ & $3(8)$ & 0,62 \\
Rechute après arrêt du traitement & & & \\
\hline
\end{tabular}

Tableau 15 : Caractéristiques à la fin du suivi des patients n'ayant pas présenté le critère de jugement principal. Les données qualitatives sont exprimées en valeur absolue et en pourcentages. Les données quantitatives sont exprimées en médiane et IQR. IQR : interquartile range. 


\subsubsection{Tolérance et effets indésirables}

Dix-huit patients du groupe biothérapie (50\%) et 15 patients du groupe immunosuppresseur conventionnel (38\%) ont présenté au moins un effet indésirable au cours du suivi $(\mathrm{p}=0,36)$. Il y avait plus d'effets indésirables infectieux chez les patients sous biothérapies (31\%), comparés aux patients traités par immunosuppresseurs conventionnels $(13 \%)$, sans que cette différence ne soit significative $(p=0,09)$ (Tableau 16). Deux patients dans chaque groupe ont développé une néoplasie $(p>0,99)$. Une réaction allergique grave et la survenue de manifestations auto-immunes paradoxales ont uniquement été recensées dans le groupe biothérapie.

Ainsi, malgré une tendance à davantage d'infections chez les patients traités par biothérapies, il n'y avait pas de différence entre les deux groupes en termes de tolérance thérapeutique, notamment concernant les effets indésirables sévères.

\begin{tabular}{|c|c|c|c|}
\hline & Biothérapie & $\begin{array}{l}\text { Immuno- } \\
\text { suppresseur }\end{array}$ & $\mathbf{p}$ \\
\hline $\begin{array}{l}\text { Nombre de patients ayant présenté au moins un } \\
\text { effet indésirable }\end{array}$ & $18(50)$ & $15(38)$ & 0,36 \\
\hline Nombre d'effets indésirables totaux & 31 & 18 & \\
\hline $\begin{array}{l}\text { Effet indésirable grave ayant nécessité l'arrêt du } \\
\text { traitement }\end{array}$ & $5(14)$ & $4(10)$ & 0,73 \\
\hline \multicolumn{4}{|l|}{ Effets indésirables infectieux } \\
\hline Infections sévères & 0 & 0 & \\
\hline Infections non graves & $11(31)$ & $5(13)$ & 0,09 \\
\hline Tuberculose & 0 & 0 & \\
\hline \multicolumn{4}{|l|}{ Effets indésirables majeurs } \\
\hline Troubles digestifs & $4(11)$ & $6(15)$ & \\
\hline réaction hépatique & $1(3)$ & $2(5)$ & \\
\hline Néoplasie & $2(6)$ & $2(5)$ & \\
\hline Syndrome dépressif & $3(8)$ & 0 & \\
\hline Manifestations auto-immunes paradoxales & $2(6)$ & 0 & \\
\hline Réaction allergique grave & $1(3)$ & 0 & \\
\hline Cytopénie & 0 & $1(3)$ & \\
\hline \multicolumn{4}{|l|}{ Effets indésirables mineurs } \\
\hline Céphalées & $3(8)$ & $1(3)$ & \\
\hline Asthénie & 0 & $2(5)$ & \\
\hline Troubles du sommeil & $2(6)$ & $1(3)$ & \\
\hline Manifestations psychiatriques (hallucinations) & $1(3)$ & 0 & \\
\hline
\end{tabular}

Tableau 16 : Effets indésirables selon le traitement étudié. Les données qualitatives sont exprimées en valeur absolue et en pourcentages. 


\subsection{Analyse de sous-groupes selon le type d'étiologie}

Quatre principaux diagnostics étiologiques ont été identifiés : 23 patients (31\%) présentaient une uvéite idiopathique, 19 (25\%) souffraient d'une maladie de birdshot, 12 (16\%) avaient pour diagnostic une sarcoïdose et enfin, 10 patients (13\%) étaient atteints d'une maladie de Behçet (Tableau 17). Les patients atteints d'une maladie de Behçet étaient les plus jeunes (33 ans [29 - 44]) et les patients atteints d'une sarcoïdose les plus âgés (56 ans [32 - 67]).

La majorité des patients présentaient une panuvéite, exceptés les patients souffrant d'une maladie de birdshot ayant, par définition, une atteinte postérieure. L'acuité visuelle initiale était la plus altérée pour les patients présentant une uvéite idiopathique. A l'inverse, l'acuité visuelle était la plus préservée chez les patients souffrant de sarcoïdose. Concernant les autres éléments de sévérité de l'uvéite, $80 \%$ des patients ayant une maladie de Behçet présentaient une vascularite active, respectivement $78 \%$ et $68 \%$ des patients souffrant d'une uvéite idiopathique ou d'une maladie de birdshot avaient un œdème maculaire à l'introduction du traitement.

Sur le plan thérapeutique, les stratégies de prise en charge différaient selon le diagnostic (Tableau 17). Les patients atteints d'une maladie de birdshot ou d'une uvéite idiopathique étaient traités dans la moitié des cas par biothérapies. La biothérapie de choix était l'adalimumab pour les patients présentant une maladie birdshot et l'adalimumab et l'infliximab pour les uvéites idiopathiques. Tous les patients ayant une uvéite de birdshot traités par immunosuppresseurs conventionnels recevaient du mycophénolate mofétil. Les uvéites idiopathiques prise en charge par immunosuppresseurs conventionnels recevaient soit du méthotrexate, soit de l'azathioprine. Soixante-dix pour cent des patients atteints d'une maladie de Behçet étaient traités par biothérapies alors que $75 \%$ des patients présentant une sarcoïdose recevaient un traitement immunosuppresseur conventionnel, en premier choix le méthotrexate.

Le critère de jugement principal a été atteint pour respectivement $74 \%$ des patients présentant une uvéite idiopathique ou une maladie de birdshot, $58 \%$ des patients atteints de sarcoïdose et $20 \%$ des patients souffrant d'une maladie de Behçet $(\mathrm{p}=0,68)$. Le délai médian de survenue du critère de jugement principal était significativement différent entre les groupes $(\mathrm{p}=0,03)$. Il était de 10 mois pour les uvéites idiopathiques, de 16 mois pour les maladies de birdshot, de 21 mois pour les uvéites sarcoïdosiques et n'était pas atteint pour les patients souffrant de maladie de Behçet (Figure 25). Une rechute, quel que soit le traitement reçu, a été observée chez $68 \%$ des patients souffrant d'une maladie de birdshot, $56 \%$ des uvéites idiopathiques, $33 \%$ des sarcoïdoses et enfin, $20 \%$ des maladies de Behçet $(p=0,05)$. Enfin, 
$25 \%$ des patients atteints de sarcoïdose ont présenté un effet indésirable sévère contre $17 \%$ des uvéites idiopathiques, $5 \%$ des patients atteints de maladie de birdshot et aucun patient souffrant de maladie de Behçet $(p=0,23)$.

La fréquence de survenue du critère de jugement principal sous immunosuppresseurs conventionnels était plus élevée chez les patients atteints d'uvéite idiopathique (82\%), suivies des maladies de birdshot (60\%), des uvéites sarcoïdosiques (56\%) et enfin des maladies de Behçet (33\%), sans que ces différences ne soient significatives $(p=0,43)$. De même, le critère de jugement principal sous biothérapies est survenu pour $89 \%$ des patients atteints de maladie de birdshot, $67 \%$ des patients présentant une uvéite idiopathique ou sarcoïdosique et enfin $33 \%$ des Behçet, à nouveau, sans différence significative $(\mathrm{p}=0,22)$. 


\begin{tabular}{|c|c|c|c|c|c|}
\hline & Idiopathique & $\begin{array}{l}\text { Maladie de } \\
\text { birdshot }\end{array}$ & Sarcoïdose & $\begin{array}{l}\text { Maladie } \\
\text { de Behçet }\end{array}$ & p \\
\hline Effectif & $23(31)$ & $19(25)$ & $12(16)$ & $10(13)$ & \\
\hline Age médian (IQR) & $48[32-69]$ & $52[41-63]$ & $56[32-67]$ & $33[29-44]$ & \\
\hline Sexe (homme) & $11(48)$ & $10(53)$ & $5(42)$ & $6(50)$ & \\
\hline \multicolumn{6}{|l|}{ Caractéristiques ophtalmologiques } \\
\hline Bilatéralité & $23(100)$ & $19(100)$ & $11(92)$ & $8(80)$ & \\
\hline \multicolumn{6}{|l|}{ Localisation uvéite } \\
\hline intermédiaire & $3(13)$ & 0 & $1(8)$ & 0 & \\
\hline postérieure & $5(22)$ & $19(100)$ & 0 & $4(40)$ & \\
\hline panuvéite & $15(65)$ & 0 & $11(92)$ & $6(60)$ & \\
\hline $\begin{array}{l}\text { Acuité visuelle (LogMAR) médiane de } \\
\text { l'œil droit (OD) [IQR] }\end{array}$ & $\begin{array}{c}0,4 \\
{[0,09-1]}\end{array}$ & $\begin{array}{c}0,3 \\
{\left[0,10^{-0,50]}\right.}\end{array}$ & $\begin{array}{c}0,15 \\
{[0,09-0,30]}\end{array}$ & $\begin{array}{c}0,3 \\
{[0-0,88]}\end{array}$ & \\
\hline $\begin{array}{l}\text { Acuité visuelle (LogMAR) médiane de } \\
\text { l'œil gauche (OG) [IQR] }\end{array}$ & $\begin{array}{c}0,22 \\
{[0,10-0,70]}\end{array}$ & $\begin{array}{c}0,3 \\
{[0,05-0,40]}\end{array}$ & $\begin{array}{c}0,1 \\
{[0,09-0,24]}\end{array}$ & $\begin{array}{c}0,1 \\
{[0-0,70]}\end{array}$ & \\
\hline Vascularite active & $8(35)$ & $6(32)$ & $1(8)$ & $8(80)$ & \\
\hline OEdème maculaire cystoïde & $18(78)$ & $13(68)$ & $7(58)$ & $4(40)$ & \\
\hline $\begin{array}{c}\text { épaisseur maculaire centrale } \\
\text { médiane }(\mu \mathrm{m} \text { en OCT) OD [IQR] }\end{array}$ & $\begin{array}{c}396 \\
{[322-454]}\end{array}$ & $\begin{array}{c}342 \\
{[323-538]}\end{array}$ & $\begin{array}{c}317 \\
{[312-420]}\end{array}$ & $\begin{array}{c}341 \\
{[323-555]}\end{array}$ & \\
\hline $\begin{array}{l}\text { épaisseur maculaire centrale } \\
\text { médiane }(\mu \mathrm{m} \text { en OCT) OG [IQR] }\end{array}$ & $\begin{array}{c}388 \\
{[322-419]}\end{array}$ & $\begin{array}{c}360 \\
{[302-547]}\end{array}$ & $\begin{array}{c}313 \\
{[303-350]}\end{array}$ & $\begin{array}{c}342 \\
{[329-355]}\end{array}$ & \\
\hline \multicolumn{6}{|l|}{ Prise en charge thérapeutique } \\
\hline Traitement par IS conventionnels & $11(48)$ & $10(53)$ & $9(75)$ & $3(30)$ & \\
\hline méthotrexate & 6 & 0 & 7 & 0 & \\
\hline azathioprine & 5 & 0 & 2 & 3 & \\
\hline mycophénolate mofétil & 0 & 10 & 0 & 0 & \\
\hline Traitement par biothérapies & $12(52)$ & $9(47)$ & $3(25)$ & $7(70)$ & \\
\hline infliximab & 5 & 1 & 2 & 5 & \\
\hline adalimumab & 6 & 7 & 1 & 2 & \\
\hline tocilizumab & 1 & 1 & 0 & 0 & \\
\hline $\begin{array}{l}\text { Durée médiane (mois) d'évolution de } \\
\text { l'uvéite [IQR] }\end{array}$ & {$[9-74]$} & {$\left[\begin{array}{c}6 \\
{[3-26]}\end{array}\right.$} & $\begin{array}{c}29 \\
{[13-53]}\end{array}$ & $\begin{array}{c}9,5 \\
{[0-25]}\end{array}$ & \\
\hline $\begin{array}{l}\text { Durée médiane (mois) d'exposition à } \\
\text { la corticothérapie }[\mathrm{IQR}]\end{array}$ & {$\left[\begin{array}{l}25 \\
{[4}\end{array}\right.$} & {$\left[\begin{array}{c}5 \\
{[3-25]}\end{array}\right.$} & {$[7-37]$} & $\begin{array}{c}9,5 \\
{[0-25]}\end{array}$ & \\
\hline Patients ayant atteint le CJP & $17(74)$ & $14(74)$ & $7(58)$ & $2(20)$ & 0,68 \\
\hline rechute & $13(56)$ & $13(68)$ & $4(33)$ & $2(20)$ & 0,05 \\
\hline effet indésirable sévère & $4(17)$ & $1(5)$ & $3(25)$ & 0 & 0,23 \\
\hline Survenue CJP sous IS conventionnels & $9(82)$ & $6(60)$ & $5(56)$ & $1(33)$ & 0,43 \\
\hline méthotrexate & 6 & 0 & 4 & 0 & \\
\hline azathioprine & 3 & 0 & 1 & 1 & \\
\hline mycophénolate mofétil & 0 & 6 & 0 & 0 & \\
\hline Survenue CJP sous biothérapies & $8(67)$ & $8(89)$ & $2(67)$ & $1(33)$ & 0,22 \\
\hline infliximab & 4 & 1 & 1 & 0 & \\
\hline adalimumab & 4 & 6 & 1 & 1 & \\
\hline tocilizumab & 0 & 1 & 0 & 0 & \\
\hline
\end{tabular}

Tableau 17 : Analyse de la prise en charge thérapeutique et du critère de jugement principal (CJP) selon l'étiologie de l'uvéite. Les données qualitatives sont exprimées en valeur absolue et en pourcentages. Les données quantitatives sont exprimées en médiane et IQR. OD : œil droit. OG : œil gauche. OCT : tomographie par cohérence optique. IQR : interquartile range. IS : immunosuppresseur. 


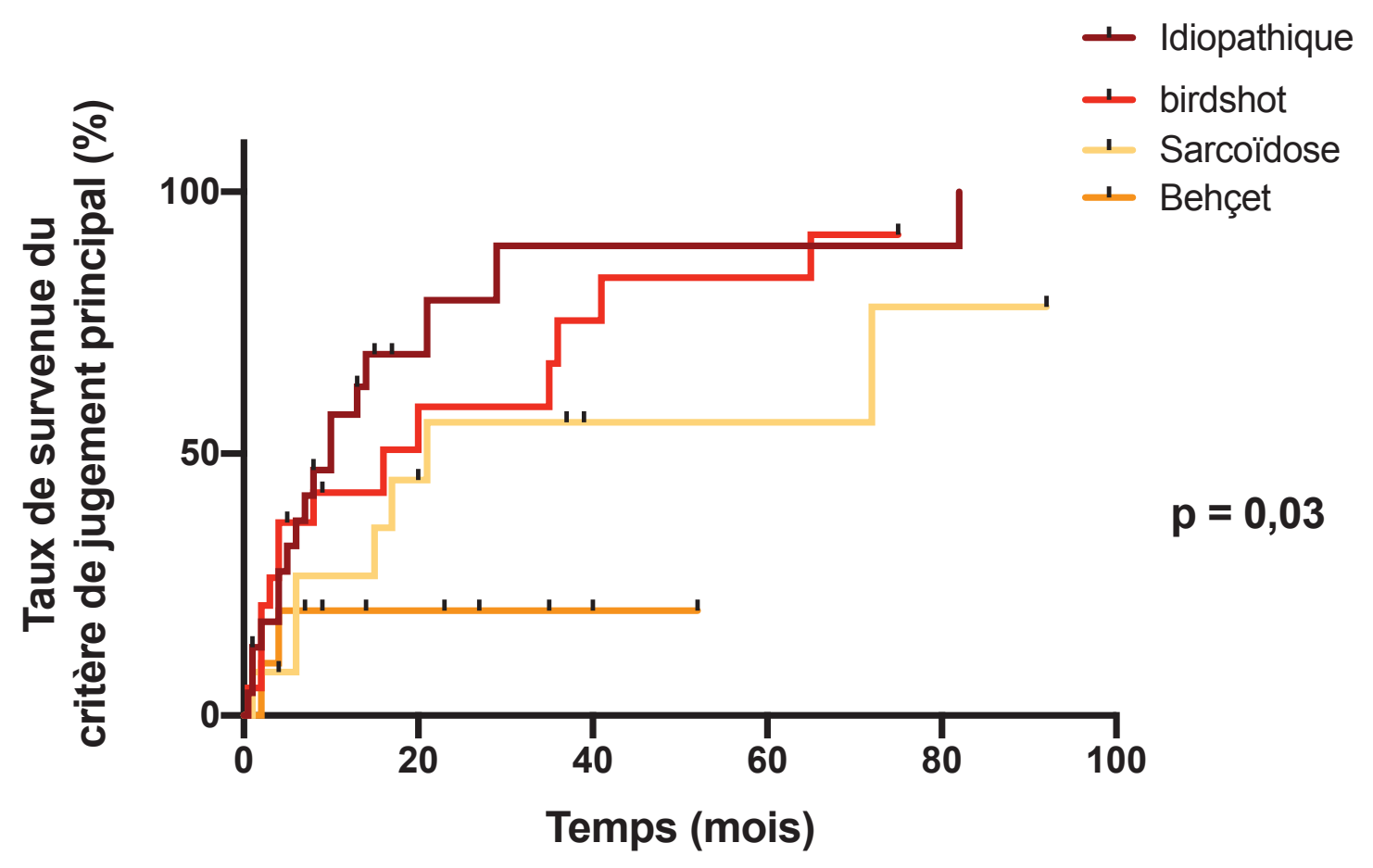

Figure 25 : Courbe de Kaplan-Meier représentant la survenue du critère de jugement principal selon le sous-groupe étiologique. Pour des raisons de lisibilités, données exprimées sur 24 mois. Les tirets verticaux représentent les données censurées.

\subsection{Facteurs associés à la prescription de biothérapie}

Afin d'argumenter et de mieux dessiner la stratégie thérapeutique actuelle, nous avons cherché à identifier des facteurs associés à la prescription de biothérapie. L'analyse univariée de différents paramètres n'a pas retrouvé de facteurs associés au recours aux biothérapies pour la prise en charge des uvéites inflammatoires (Tableau 18). 


\begin{tabular}{|c|c|c|c|c|}
\hline & Biothérapie & $\begin{array}{c}\text { Immuno- } \\
\text { suppresseur }\end{array}$ & $\begin{array}{l}\text { OR (intervalle de } \\
\text { confiance } 95 \% \text { ) }\end{array}$ & $\mathbf{p}$ \\
\hline \multicolumn{5}{|l|}{$\begin{array}{l}\text { Caractéristiques générales de la } \\
\text { population }\end{array}$} \\
\hline Sexe (homme) & $22(61)$ & $15(38)$ & $\begin{array}{c}2,51 \\
(0,99-6,37)\end{array}$ & 0,08 \\
\hline Age au diagnostic & & & & 0,31 \\
\hline$<30$ ans & $8(22)$ & $8(21)$ & référence & \\
\hline 30 ans $\leq$ âge $\leq 50$ ans & $19(53)$ & $15(38)$ & $\begin{array}{c}1,27 \\
(0,38-4,23)\end{array}$ & \\
\hline$>50$ ans & $9(25)$ & $16(41)$ & $\begin{array}{c}0,56 \\
(0,15-2,01)\end{array}$ & \\
\hline \multicolumn{5}{|l|}{ Etiologie de l'uvéite } \\
\hline Idiopathique & $12(33)$ & $11(28)$ & $\begin{array}{c}1,27 \\
\left(0,48^{-}-3,40\right)\end{array}$ & 0,8 \\
\hline Maladie de birdshot & $9(25)$ & $10(26)$ & $\begin{array}{c}0,97 \\
(0,34-2,74)\end{array}$ & $>0,99$ \\
\hline Maladie de Behçet & $7(19)$ & $3(8)$ & $\begin{array}{c}2,90 \\
(0,69-12,20)\end{array}$ & 0,18 \\
\hline Sarcoïdose & $3(8)$ & $9(23)$ & $\begin{array}{c}0,30 \\
(0,07-1,23)\end{array}$ & 0,12 \\
\hline \multicolumn{5}{|l|}{$\begin{array}{l}\text { Caractéristiques ophtalmologiques au } \\
\text { diagnostic }\end{array}$} \\
\hline Localisation uvéite & & & $\begin{array}{c}0,83 \\
(0,10-6,65)\end{array}$ & $>0,99$ \\
\hline intermédiaire & $2(6)$ & $2(5)$ & & \\
\hline postérieure & $18(50)$ & $15(38)$ & & \\
\hline panuvéite & $16(44)$ & $22(56)$ & & \\
\hline $\begin{array}{l}\text { Acuité visuelle bilatérale au diagnostic } \geq 0,4 \\
\text { LogMAR }\end{array}$ & $8(22)$ & $16(41)$ & $\begin{array}{c}0,44 \\
(0,16-1,23)\end{array}$ & 0,13 \\
\hline $\begin{array}{l}\text { Acuité visuelle bilatérale au diagnostic } \geq 1 \\
\text { LogMAR }\end{array}$ & $2(6)$ & $6(15)$ & $\begin{array}{c}0,34 \\
(0,06-1,84)\end{array}$ & 0,27 \\
\hline $\begin{array}{l}\text { Acuité visuelle unilatérale au diagnostic } \geq 0,4 \\
\text { LogMAR }\end{array}$ & $19(53)$ & $26(67)$ & $\begin{array}{c}0,62 \\
(0,23-1,68)\end{array}$ & 0,45 \\
\hline $\begin{array}{l}\text { Acuité visuelle unilatérale au diagnostic } \geq 1 \\
\text { LogMAR }\end{array}$ & $6(17)$ & $13(33)$ & $\begin{array}{c}0,43 \\
(0,14-1,30)\end{array}$ & 0,18 \\
\hline Inflammation intraoculaire active & $24(67)$ & $28(72)$ & $\begin{array}{c}0,86 \\
\left(0,27^{-}-2,63\right)\end{array}$ & $>0,99$ \\
\hline Vascularite active & $18(50)$ & $21(54)$ & $\begin{array}{c}0,43 \\
(0,12-1,49)\end{array}$ & 0,23 \\
\hline CEdème papillaire & $14(39)$ & $11(28)$ & $\begin{array}{c}1,75 \\
(0,63-4,85)\end{array}$ & 0,31 \\
\hline $\begin{array}{l}\text { CEdème maculaire cystoïde } \\
\text { Caractéristiques thérapeutiques lors de la } \\
\text { rechute }\end{array}$ & $17(47)$ & $24(62)$ & $\begin{array}{c}0,71 \\
(0,26-1,90)\end{array}$ & 0,62 \\
\hline $\begin{array}{l}\text { Posologie de prednisone lors de la première } \\
\text { rechute } \geq 10 \mathrm{mg}\end{array}$ & $18(50)$ & $24(62)$ & $\begin{array}{c}0,58 \\
(0,23-1,48)\end{array}$ & 0,35 \\
\hline $\begin{array}{l}\text { Posologie de prednisone lors de la première } \\
\text { rechute } \geq 25 \mathrm{mg}\end{array}$ & $8(22)$ & $9(23)$ & $\left(0,31^{0,92}-2,72\right)$ & $>0,99$ \\
\hline
\end{tabular}

Tableau 18 : Facteurs associés à la prescription de biothérapies (analyse univariée). Les données qualitatives sont exprimées en valeur absolue et en pourcentages. Les résultats sont exprimés en oddratios avec intervalle de confiance à $95 \%$. 


\section{Discussion}

Actuellement, le traitement des uvéites non-infectieuses non-antérieures reste un traitement suspensif et non curatif. Les différentes options thérapeutiques en cas de contrôle insuffisant sous corticothérapie ou de cortico-dépendance sont bien identifiées : immunosuppresseurs conventionnels ou biothérapies (64). La question persistante est celle de leur hiérarchisation au sein de la stratégie thérapeutique.

A notre connaissance, il s'agit de la première étude rétrospective comparant le risque de récidive et les effets indésirables graves sous immunosuppresseurs conventionnels versus biothérapies dans les uvéites non-infectieuses non-antérieures.

Nous avons fait le choix d'utiliser un critère de jugement composite. Par ce critère, nous avons cherché à évaluer à la fois l'efficacité du traitement et sa tolérance, nous permettant ainsi de nous approcher des conditions de «vie réelle " de prescription, à travers les questions d'intensification thérapeutique ou d'arrêt du traitement.

Les critères permettant de diagnostiquer la rechute, ou encore les critères d'amélioration de l'acuité visuelle n'ont pas été définis lors du travail de nomenclature de l'équipe du SUN (3). L'évaluation de ces paramètres est hétérogène dans la littérature, limitant les possibilités de comparaison entre les différentes études. La deuxième limite dans cette comparaison repose sur l'hétérogénéité des patients au sein des différentes cohortes d'uvéites, devant des diagnostics étiologiques variés. Ce choix non restrictif d'inclusion, utilisé pour les études prospectives VISUAL I (52) et II (53), est communément admis, en lien avec la faible prévalence des différents diagnostics et donc du risque d'effectif réduit.

\subsection{Analyses des résultats : groupe biothérapie}

Vingt patients ont atteint le critère de jugement principal. Il s'agit d'un taux élevé, correspondant à $56 \%$ des patients. Le délai médian de survenue de ce critère était relativement court (16 mois). Quinze patients (41\%) ont présenté une rechute, pour une durée médiane de suivi de 9,5 mois. Ce résultat est en cohérence avec les données de la littérature. En effet, le taux de rechute sous adalimumab dans l'étude VISUAL II (53) était de $39 \%$, pour une durée médiane de suivi de 245 jours, soit environ 8 mois. Dans une étude prospective incluant 131 patients traités par adalimumab dont 44 uvéites antérieures, Díaz-Llopis et al. (65) ont retrouvé des taux de rechutes similaires aux nôtres : 38,2 \% à 6 mois et $42 \%$ à 1 an. A l'inverse, 
Fabiani et al. (66) ont retrouvé un taux de rechute de $21 \%$ sous anti-TNF alpha, soit bien inférieur au nôtre, mais la définition de la rechute dans leur travail était laissée au libre choix du praticien, exposant au risque de minorer le nombre de rechutes.

Le premier paramètre signant la rechute dans notre étude était l'absence d'amélioration ou l'apparition d'un œdème maculaire cystoïde. Dans l'étude VISUAL I (52), le premier paramètre signant la rechute était la majoration de l'inflammation au niveau du segment antérieur, critère le moins fréquemment retrouvé dans notre étude. Une des explications possibles est que notre étude incluait à la fois des uvéites actives et des uvéites non actives cortico-dépendantes. On peut alors émettre l'hypothèse que les uvéites actives présentent un risque de récidive d'inflammation antérieure plus élevé que les uvéites cortico-dépendantes.

Le taux de survie sans progression dans notre étude était plus faible que celui de Vallet et al. (67) : $90 \%$ à 6 mois dans leur travail versus 70,4 \% dans notre étude, $70 \%$ à 12 mois versus $60,4 \%$ et $59 \%$ à 24 mois dans leur travail versus $43,7 \%$ dans notre étude. Certes, l'étude de Vallet et $a l$. a inclus un nombre de patients plus élevé que la nôtre et apparait donc comme plus puissante. Cependant, notre définition de la rechute était plus précise puisque dans leur travail, la rechute était définie comme l'apparition d'une nouvelle lésion oculaire et/ou l'aggravation d'une lésion préexistante, nécessitant une intensification thérapeutique. La dégradation de l'acuité visuelle ou l'apparition d'un œdème maculaire ne faisaient pas partie des paramètres inclus dans leur définition. Nous avons choisi une définition précise de la rechute, reposant sur des critères objectifs de l'examen ophtalmologique, basée sur la définition utilisée dans les études VISUAL I (52) et II (53). De plus, nous avons inclus dans le critère de rechute l'absence d'amélioration ou l'apparition d'un œdème maculaire sous traitement. Ainsi, notre critère était plus « sévère » et a donc probablement augmenté notre taux de rechute. Nous avons fait ce choix pour nous approcher des conditions de vie réelle de prescription et d'intensification thérapeutique. En pratique clinique, malgré le manque de recommandation, l'absence d'amélioration de l'œdème maculaire sous traitement va engendrer une sanction thérapeutique. Ce paramètre pourrait être inclus dans la définition de la rechute dans de futures études prospectives.

Enfin, la population d'étude de Vallet et al. et la nôtre n'étaient pas similaires : $36 \%$ de leurs patients souffraient d'une maladie de Behçet, seuls $14 \%$ des uvéites étaient idiopathiques et la proportion d'uvéite de birdshot n'était pas précisée. Ces variations étiologiques peuvent influencer le taux de rechute. Dans notre étude de sous-groupes, nous avons relevé que les patients atteints d'uvéites idiopathiques avaient plus fréquemment présenté le critère de 
jugement principal et que leur délai de survenue du critère de jugement principal était statistiquement plus court. De plus, d'un point de vue physiopathologique, les lymphocytes Th1 apparaissent comme jouant un rôle indéniable au cours de la maladie de Behçet (68), alors que les lymphocytes Th17 sont les premiers acteurs de la maladie de birdshot (69).

Cependant, qu'il s'agisse de notre étude ou de celle de Vallet et al., le taux de survie sans progression reste relativement faible après deux ans de traitement, entre 45 et $60 \%$, signant ainsi la sévérité des uvéites et leur risque élevé de récidive.

Une amélioration de l'acuité visuelle a été observée chez $15 \%$ des patients après 6 mois de traitement et $31 \%$ des patients après 12 mois de traitement. On peut d'abord légitimement s'interroger sur l'interprétation de ce pourcentage : doit-il être considéré comme élevé ou, à l'inverse, comme décevant? Il faut alors rappeler que $70 \%$ des patients n'ont pas répondu à un traitement immunosuppresseur conventionnel antérieurement. Il s'agit donc de patients sévères, pour lesquels le pronostic visuel, en l'absence de biothérapie, aurait probablement était très péjoratif.

Dans une étude rétrospective française portant sur 21 patients (70), une amélioration de l'acuité visuelle était identifiée dans $45,1 \%$ des cas à 6 mois et 52,9\% des cas à 12 mois. Ce taux était plus élevé que dans notre étude mais l'amélioration était définie différemment : amélioration de 2 lignes sur l'échelle ETDRS (versus 3 dans notre travail). Les auteurs ont considéré des patients en amélioration là où nous avons conclu à une stabilité. Nous avons choisi ce chiffre de 3 lignes car il s'agissait de la définition retenue dans les études VISUAL. Enfin, une méta-analyse de 2018 a montré une amélioration de l'acuité visuelle d'au moins 3 lignes dans $41,3 \%$ des cas, et une amélioration de 2 lignes pour 51,5\% des cas (71). Toutefois, cette méta-analyse comportait des uvéites antérieures, des uvéites pédiatriques et ne portait que sur l'adalimumab.

Notre étude a mis en exergue l'efficacité remarquable des biothérapies dans le contrôle rapide et prolongé de l'inflammation oculaire. L'état de quiescence était observé dans $76 \%$ des cas après 1 mois de traitement, $92 \%$ des cas à 6 mois puis $71 \%$ des cas à un an. Ces résultats ont déjà été montrés dans la littérature. Martel et al. (72), ont constaté un état de quiescence pour $82,1 \%$ des malades sous infliximab à 6 mois de traitement, $69,6 \%$ à 12 mois et pour $75 \%$ des malades sous adalimumab à 6 mois de traitement et 51,2\% à 12 mois. La définition de l'état de quiescence était identique à la nôtre. L'étude VISUAL III (73) est une étude prospective où ont été inclus l'ensemble des patients des études VISUAL I et II (371 patients), du groupe 
placebo ou traité, ayant présenté ou non une rechute. Au début de l'étude, $34 \%$ des patients avaient un état oculaire quiescent. Ce taux a été augmenté à $86 \%$ après 78 semaines de traitement. Notre étude confirme les données de la littérature quant à l'efficacité rapide des biothérapies sur le contrôle de l'inflammation oculaire.

Notre étude a montré une régression de l'œdème maculaire initial chez $50 \%$ des patients. Il s'agit donc d'une efficacité qui reste partielle. Les données de la littérature sont divergentes concernant l'efficacité des anti-TNF alpha dans le traitement de l'œdème maculaire. Dans une étude rétrospective française portant sur 25 patients (74), une résolution de l'œdème maculaire était observée dans $50 \%$ des cas sous adalimumab et $56 \%$ des cas sous infliximab, après 24 mois de traitement. Díaz-Llopis et al. (65) retrouvaient une résolution complète de l'œdème maculaire dans $70 \%$ des cas après 6 mois de traitement. En revanche, dans l'étude VISUAL III, une stabilité de l'épaisseur maculaire centrale était notée au cours du suivi.

De façon très intéressante, l'évolution de l'œdème maculaire sous traitement n'a pas été étudiée dans les études VISUAL I (52) et II (53). Dans ces deux études, le risque de développer un œdème maculaire sous adalimumab était significativement diminué par rapport au groupe placebo. En revanche, il n'était absolument pas précisé l'évolution de l'œdème maculaire sous traitement. Il s'agit pourtant d'un élément pronostic majeur. De futures études prospectives devront s'attacher à l'évolution de l'œdème maculaire cystoïde sous anti-TNF alpha.

Notre étude a mis en évidence une décroissance statistiquement significative de la corticothérapie sous biothérapies. A 6 mois de traitement, la posologie médiane quotidienne de corticoïdes était de $11 \mathrm{mg}$ et à 12 mois elle était de $7 \mathrm{mg}$. Ces résultats sont en cohérence avec les données de la littérature. L'équipe de Vallet et al. (67) retrouvait une décroissance similaire de la corticothérapie : posologie médiane initiale de 20 mg, 10 mg à 6 mois et 7 mg à 12 mois. Trente-huit pour cent de nos patients bénéficiaient d'une posologie de corticoïdes $<10 \mathrm{mg}$ à 6 mois de traitement, similaires aux résultats retrouvés par Maleki et al. (75). En revanche, notre taux de sevrage en corticoïdes était très inférieur (22\%) au résultat de la méta-analyse de Ming et $a l$. (71) où $48,8 \%$ des patients avaient été sevrés en corticoïdes. Ce pourcentage a été obtenu à partir des données de 6 études, dont 4 portaient sur la prise en charge des uvéites au cours de l'arthrite juvénile idiopathique, exclues de notre travail, limitant ainsi les possibilités de comparaison.

Ainsi, les biothérapies présentent une efficacité significative en termes d'épargne cortisonique. Cependant, qu'il s'agisse de notre étude ou de celle de Vallet et al., les posologies 
de corticoïdes sont restées élevées, de l'ordre de $10 \mathrm{mg}$ à 6 mois de traitement. Comment expliquer ce résultat? Suite à l'étude VISUAL I (52), portant sur les uvéites actives, il est apparu qu'une décroissance trop rapide de la corticothérapie semblait exposer à un risque élevé de rechute et donc, qu'une décroissance lente était nécessaire. Dans le cadre de l'étude VISUAL II portant sur les uvéites inactives cortico-dépendantes, le schéma de décroissance de la corticothérapie était accéléré par rapport à la pratique clinique (sevrage en 19 semaines) (53). Or, le taux de rechute dans cette étude était similaire au nôtre. Ainsi, malgré un sevrage en corticoïdes plus précoce ( $<6$ mois), les patients souffrant d'une uvéite inactive corticodépendante issus de l'étude VISUAL II ont présenté un taux de rechute identique à celui de notre étude. Une décroissance accélérée des corticoïdes pour ces patients particuliers, lors d'un traitement par adalimumab, n'exposerait pas à un risque de rechute majoré.

Cinq patients $(14 \%)$ sous biothérapies ont présenté un effet indésirable grave ayant nécessité d'interrompre le traitement. Ces chiffres sont en cohérence avec les trois études VISUAL : $11,7 \%(52), 9,6 \%$ (53) et $19 \%$ (73). Ce dernier chiffre de $19 \%$ (VISUAL III) est légèrement plus élevé que le nôtre, en lien avec une inclusion dans les causes d'arrêt du traitement pour effet indésirable, l'apparition d'un œdème maculaire. Il s'agissait de la deuxième cause d'arrêt du traitement, soulignant à nouveau l'importance de ce paramètre dans l'évaluation du contrôle de l'uvéite. La première cause d'arrêt du traitement dans les études VISUAL II et III était la mise en évidence biologique de Mycobacterium tuberculosis, avec ou sans signe clinique. Aucun patient n'a présenté d'infection tuberculeuse au cours de notre étude.

Dix-huit patients $(50 \%)$ ont présenté au moins un effet indésirable au cours du suivi, principalement de type infectieux (31\%). Ce nombre d'effets indésirables totaux était plus élevé dans notre étude que pour Vallet et al. (28\%) (67) ou Mercier et al. (29\%) (70). La prise en compte dans notre nombre d'effets secondaires infectieux des infections bénignes ambulatoires, peut expliquer cette différence qui se réduit secondairement après exclusion de ces affections à $36 \%$ (13 patients). Aucune infection sévère n'est survenue.

Nous avons retrouvé un taux de néoplasie (6\%) plus élevé dans notre travail que dans l'étude de Vallet et al. (2,5\%) (67) ou que les études VISUAL I et II (1,8\% et 0,87\%) (52,53). Le manque de puissance de notre étude peut avoir artificiellement majoré le taux de néoplasie. En revanche, notre durée de suivi était plus longue que dans les études VISUAL, mettant ainsi en relief l'importance de surveiller le risque néoplasique sur le long terme chez ces patients.

De manière inattendue, nous avons détecté 3 syndromes dépressifs ayant nécessité l'arrêt du traitement pour 2 patients. Il s'agit d'un effet indésirable peu décrit dans la littérature, 
uniquement sous forme de case reports avec des facteurs confondants associés (76). Cependant, dans l'étude VISUAL I, l'apparition d'idées suicidaires avait nécessité également l'arrêt du traitement (52). A l'inverse, une amélioration des symptômes dépressifs observés lors des maladies chroniques, notamment le psoriasis, a été décrite sous anti-TNF alpha (77). Cet effet indésirable est peut-être lié à la pathologie oculaire en elle-même, et non uniquement imputable au traitement, secondaire au handicap majeur que représente une altération de la vision. Cependant, au vu de nos résultats, l'introduction d'un traitement anti-TNF alpha devra se faire avec prudence chez les patients dépressifs.

Notre étude de sous-groupes, sous la réserve inhérente de son manque de puissance, a montré une supériorité significative de l'infliximab comparé à l'adalimumab et le tocilizumab en termes de prévention des rechutes. Le délai médian de survenue du critère de jugement principal était plus long sous infliximab (28 mois) que sous adalimumab (10 mois) $(\mathrm{p}=0,22)$. Deux études apportent des résultats divergents sur la supériorité de l'infliximab versus l'adalimumab. Vallet et al. (67) retrouvaient une tendance à la supériorité de l'adalimumab en termes de survie sans évènement $(\mathrm{p}=0,08)$, en corolaire d'une tendance à davantage d'effets indésirables sous infliximab. A l'inverse, Fabiani et al. (78) ont observé une tendance à la supériorité de l'infliximab concernant l'épargne cortisonique et la réduction de l'œdème maculaire. Des études prospectives randomisées seront nécessaires pour conclure définitivement.

Assurant une amélioration de l'acuité visuelle pour un tiers des patients après un an de traitement et présentant un pouvoir d'épargne cortisonique certain, les biothérapies apparaissent comme des thérapeutiques efficaces dans la prise en charge des uvéites non-infectieuses nonantérieures actives ou cortico-dépendantes, ayant échappé, dans $70 \%$ des cas, à un traitement immunosuppresseur conventionnel. Leur principal point fort est une rapidité d'action dans le contrôle de l'inflammation oculaire, tant du segment antérieur que du vitré. Cependant, le nombre de rechutes reste élevé avec une survie sans progression limitée à 43,7\% des patients après 2 ans de traitement. De plus, une attention particulière doit être apportée aux risques infectieux et néoplasiques. Enfin, leur efficacité dans le contrôle de l'œdème maculaire reste modérée, puisqu'il s'agit du premier paramètre ophtalmologique signant la rechute. 


\subsection{Analyses des résultats : groupe immunosuppresseur conventionnel}

Vingt-trois patients (59\%) ont atteint le critère de jugement principal. Le délai médian de survenue du critère de jugement principal était de 21 mois. Le taux de survie sans progression s'élevait à 71,7 \% après 6 mois de traitement, 60,3\% après un an et 46,5\% après 2 ans de traitement. Nous n'avons pas identifié de publication portant sur le taux de survie sans progression chez les patients présentant une uvéite non-antérieure traitée par immunosuppresseur conventionnel. La survie sans progression a diminué de $25 \%$ entre le $6^{\text {ème }}$ et le $24^{\text {ème }}$ mois de suivi. Ainsi, tout comme pour les patients traités par biothérapies, nos résultats pour les patients traités par immunosuppresseurs conventionnels rappellent le fort risque de récidive des uvéites et le caractère non curatif des traitements.

Dix-neuf patients ont présenté une rechute (49\%), pour une durée médiane de suivi de 13 mois. L'analyse de sous-groupes a mis en évidence un taux de rechute de $71 \%$ pour les patients traités par méthotrexate, versus $60 \%$ sous mycophénolate mofétil et $20 \%$ des patients recevant de l'azathioprine $(\mathrm{p}=0,02)$. Les principales données de la littérature portant sur l'efficacité des immunosuppresseurs conventionnels au cours des atteintes inflammatoires oculaires sont issues de la cohorte SITE (47). Toutefois, le taux de rechute n'était pas analysé dans ce travail. Le paramètre approchant était l'inefficacité du traitement. L'efficacité était définie selon le contrôle de l'inflammation oculaire et l'épargne cortisonique. Ainsi, les auteurs retenaient un taux d'inefficacité dans l'année suivant l'introduction du traitement de 15,5\% sous méthotrexate, $13,1 \%$ sous mycophénolate mofétil et 17,4 \% sous azathioprine. La définition différente du paramètre étudié peut expliquer la divergence entre ces données et nos résultats. De plus, ces pourcentages sont issus de l'analyse de la population dans son entier, incluant des sclérites, des uvéites antérieures et d'autres maladies inflammatoires oculaires. Des taux de rechute plus faibles ont été décrits dans une étude prospective randomisée (79), portant sur le mycophénolate sodium (41\%) ou encore pour l'azathioprine (12\%, avec un délai médian de 8 mois) (80). Le principal biais rétrospectif et le manque d'effectif ont pu surestimer notre taux de rechute.

Le taux de rechute était plus élevé chez les patients traités par méthotrexate. Rathinam et al. (81), dans une étude prospective, randomisée, multicentrique, portant sur 67 patients, ont comparé l'efficacité du mycophénolate moféil et du méthotrexate. A 6 mois, le taux d'inefficacité était de $20 \%$ dans le groupe méthotrexate et $47 \%$ dans le groupe mycophénolate mofétil. Ces résultats divergents s'expliquent, en partie, par une répartition étiologique différente de notre travail. Dans leur étude, le diagnostic étiologique le plus fréquent 
était la maladie de Vogh-Koyanagi-Harada et il n'y avait aucune maladie de birdshot. Une étude de 2019 de Gangaputra et al. (82) a retrouvé des résultats similaires à notre étude de sousgroupes, montrant une supériorité du mycophénolate mofétil concernant le contrôle de l'inflammation et l'épargne cortisonique.

Le traitement par immunosuppresseurs conventionnels a permis d'améliorer l'acuité visuelle chez $10 \%$ des patients après 6 mois de traitement et de la stabiliser chez $83 \%$. Après 12 mois de traitement, une amélioration de l'acuité visuelle était retrouvée chez $12 \%$ des patients et une stabilité chez $82 \%$. Samson et al. (83), dans une étude rétrospective portant sur 160 patients traités par méthotrexate, ont montré une amélioration ou une stabilité de l'acuité visuelle dans $91 \%$ des cas à la fin de l'étude (durée moyenne de 16 mois). Plusieurs études ont montré une amélioration de la variation de l'acuité visuelle à partir des chiffres médians d'acuité $(81,84)$. Notre étude est l'une des rares à avoir montré une amélioration de l'acuité visuelle selon une définition précise, et non uniquement sur une variation du chiffre médian. Cependant, une amélioration n'a été notée que dans $10 \%$ des cas. Les traitements immunosuppresseurs conventionnels vont permettre de limiter la dégradation de l'acuité visuelle inéluctable en l'absence de traitement, mais vont être peu efficaces dans la récupération d'une acuité visuelle déjà altérée.

Dans notre étude, à 6 mois de traitement, $68 \%$ des patients présentaient un état de quiescence inflammatoire oculaire et ce chiffre s'élevait à $76 \%$ à un an. Nos résultats apparaissent en cohérence avec les éléments de la littérature. Les données issues de la cohorte SITE ont retrouvé un contrôle de l'inflammation oculaire après un an de traitement pour $75 \%$ des uvéites intermédiaires et $52 \%$ des uvéites postérieures et panuvéites sous méthotrexate (48). Sous mycophénolate mofétil, $77 \%$ des uvéites intermédiaires et $71 \%$ des uvéites postérieures et panuvéites (49) étaient contrôlées, contre respectivement $90 \%$ et 59,7 \% sous azathioprine (50). Dans la plus grande cohorte rétrospective comparative, le taux de succès thérapeutique sur le contrôle de l'inflammation oculaire à 6 mois était plus faible pour le méthotrexate (42\%) versus $58 \%$ sous azathioprine et $70 \%$ avec le mycophénolate mofétil (51). L'efficacité des traitements immunosuppresseurs conventionnels a été montrée dans la littérature et le mycophénolate mofétil apparaît comme le plus efficace pour contrôler l'inflammation. 
Dans notre travail, l'œdème maculaire a été corrigé chez $48 \%$ des patients. Dans l'étude de Rathinam et al. (81), ce taux était plus élevé avec une correction de l'œdème maculaire dans $77 \%$ des cas sous méthotrexate et $54 \%$ sous mycophénolate mofétil. Dans un autre travail portant sur le mycophénolate mofétil (84), une résolution complète de l'œdème maculaire était obtenue chez $50 \%$ des patients, une résolution initiale puis une rechute dans $17 \%$ des cas et une absence d'amélioration dans $33 \%$ des cas. Enfin, Mackensen et al. (85) ont retrouvé une résolution de l'œdème maculaire cystoïde après 3 mois de traitement par méthotrexate chez $25 \%$ des patients. L'efficacité des immunosuppresseurs conventionnels dans la résolution de l'œdème maculaire reste partielle.

Notre étude a mis en évidence une décroissance statistiquement significative de la corticothérapie sous immunosuppresseurs conventionnels. A 12 mois de traitement, la posologie de corticoïdes était $\leq 10 \mathrm{mg} /$ jour pour $78 \%$ des patients et $\leq 5 \mathrm{mg} /$ jour pour $22 \%$. Cinq patients (13\%) ont pu être sevrés en corticoïdes. Concernant ce dernier paramètre, l'étude rétrospective de Galor (51), indiquait un arrêt de la corticothérapie après 6 mois de traitement dans $5,7 \%$ des cas sous méthotrexate, $12 \%$ sous mycophénolate mofétil et 5,8\% sous azathioprine. Concernant les données issues de la cohorte SITE, après un an de traitement par méthotrexate (48), mycophénolate mofétil (49) et azathioprine (50), respectivement $48 \%, 57 \%$ et $50 \%$ des patients recevaient une posologie de corticoïdes $\leq 10 \mathrm{mg} / \mathrm{jour}$ et respectivement $40 \%, 40 \%$ et $49 \%$ des patients avaient $\leq 5 \mathrm{mg}$ jour de corticoïdes. Ainsi, notre étude a retrouvé un taux plus élevé de patients recevant moins de $10 \mathrm{mg} /$ jour de corticoïdes mais un taux moins élevé de patients recevant moins de $5 \mathrm{mg} /$ jour de corticoïdes. Une des interprétations de ce résultat peut être liée à une différence de pratique dans la décroissance de la corticothérapie entre les équipes françaises et américaines. Par exemple, dans la vascularite à cellules géantes, les habitudes de décroissance de la corticothérapie sont plus rapides pour les équipes américaines que françaises (86).

Quatre patients $(10 \%)$ ont présenté un effet indésirable grave ayant nécessité d'interrompre le traitement. Quinze patients (38\%) ont présenté au moins un effet indésirable au cours du suivi, majoritairement d'ordre digestif (15\%) ou infectieux (13\%). Dans la cohorte SITE, durant la première année de traitement, $16 \%$ des patients (toutes étiologies confondues, y compris les autres causes d'inflammation oculaire) ont arrêté le méthotrexate pour effets indésirables (48), $13 \%$ ont arrêté le mycophénolate mofétil (49) et $24 \%$ l'azathioprine (50). Les effets indésirables étaient également majoritairement d'ordre digestif. Samson et al. (83), 
étudiant uniquement le méthotrexate, ont retrouvé $18 \%$ d'effets indésirables sévères ayant nécessité l'arrêt du traitement et un total d'effets indésirables de $43 \%$. Dans l'étude de Rathinam, $80 \%$ des patients dans chaque groupe (méthotrexate ou mycophénolate mofétil) développaient au moins un effet indésirable (81). Notre étude a retrouvé un taux d'effets indésirables total et nécessitant l'arrêt du traitement inférieurs aux données de la littérature. Une des explications peut être en lien avec la posologie des traitements. Notre posologie médiane de méthotrexate était de 13,75 mg contre $25 \mathrm{mg}$ dans l'étude de Rathinam. Ainsi, aux prix d'une moindre efficacité, une posologie réduite d'immunosuppresseur conventionnel semblerait limiter les effets indésirables.

Traitements historiques de première intention en l'absence de contrôle de l'inflammation oculaire sous corticoïdes seuls ou de cortico-dépendance, les immunosuppresseurs conventionnels apparaissent comme efficaces sur l'épargne cortisonique et le contrôle de l'inflammation oculaire. En revanche, ces thérapeutiques ne permettent pas une amélioration de l'acuité visuelle mais plutôt une stabilisation de celle-ci. La moitié des patients ont présenté une rechute, rappelant ainsi le fort risque de récidive de ces pathologies et la nécessité de thérapeutiques alternatives. Le méthotrexate semble être le traitement le plus à risque de rechute. De plus, un contrôle de l'œdème maculaire n'a été permis que dans $48 \%$ des cas, soulignant la limite de ces thérapeutiques. Cependant, les immunosuppresseurs conventionnels apparaissent comme des traitements présentant une sûreté d'utilisation, notamment sur le plan néoplasique. Les effets indésirables, majoritairement digestifs, ont évolué favorablement à l'arrêt du traitement.

\subsection{Comparaison des deux stratégies thérapeutiques}

De manière inhérente à notre méthode de constitution des groupes, nous avons observé une différence statistiquement significative entre les deux groupes concernant l'exposition à un traitement immunosuppresseur antérieur $(p=0,03)$. De plus, les patients du groupe biothérapie présentaient une durée d'évolution de la maladie plus longue $(p=0,03)$, une exposition à la corticothérapie allongée $(p=0,03)$ et une maladie ayant plus fréquemment récidivée $(p=0,01)$. Nous avons fait ce choix pour nous approcher des conditions réelles de prescription des biothérapies au cours des uvéites non-infectieuses. Cette prescription relève actuellement d'une prescription de deuxième intention, exceptée dans la maladie de Behçet en cas de menace pour le pronostic visuel (64). En revanche, à l'introduction du traitement, il n'y avait pas de différence 
entre les deux groupes concernant le degré d'inflammation oculaire, l'altération de l'acuité visuelle, la présence d'une vascularite ou d'un œdème maculaire cystoïde, nous autorisant ainsi une comparaison pour l'épisode actuel.

Nous n'avons pas mis en évidence de différence significative entre les deux groupes concernant notre critère de jugement principal. Dans le groupe biothérapie, le taux de survie sans progression se portait à $97,2 \%$ à 1 mois, 70,4 \% à 6 mois, $60,4 \%$ à 1 an et $43,7 \%$ à 2 ans de traitement. Dans le groupe immunosuppresseur conventionnel, ces pourcentages s'élevaient à $89,7 \%$ à 1 mois, $71,7 \%$ à 6 mois, $60,3 \%$ à 1 an et $46,5 \%$ à 2 ans de traitement. Le délai médian de survenue du critère de jugement principal était plus long dans le groupe immunosuppresseur conventionnel (21 mois versus 16 mois, $p=0,47$ ).

Peu d'études ont comparé ces deux stratégies. Abásolo et al. ont comparé de manière rétrospective l'efficacité thérapeutique des immunosuppresseurs conventionnels et des biothérapies (87). Contrairement à notre travail, il n'a pas été défini de critères de rechute mais d'efficacité. L'amélioration clinique était laissée à la libre appréciation du clinicien. La répartition étiologique était différente par rapport à notre étude avec une majorité d'uvéites idiopathiques, peu de sarcoïdoses et une inclusion d'uvéites antérieures. Enfin, seuls $8 \%$ des patients bénéficiaient d'anti-TNF alpha en monothérapie (incluant l'étanercept et le golimumab) et $17,9 \%$ de bithérapie associée à un immunosuppresseur conventionnel $(8,9 \%$ dans notre étude). Ces limites étant posées, il est intéressant de relever que cette étude n'a pas mis en évidence de différence statistique entre le méthotrexate, l'azathioprine, la ciclosporine et les anti-TNF alpha en termes d'arrêt du traitement pour inefficacité.

Comment expliquer, dans notre étude, l'absence de différence significative entre les deux groupes en termes de survie sans progression ? Il faut commencer par rappeler que les patients traités par biothérapies avaient déjà, pour la majorité d'entre eux (70\%), présenté une rechute sous immunosuppresseurs conventionnels. De ce fait, il s'agissait de patients plus sévères, ayant déjà résisté à un traitement de deuxième intention et donc plus à risque de rechuter à nouveau. En l'absence de prescription de biothérapies, devant l'échec d'une voire deux lignes de traitement immunosuppresseur, on peut estimer que le pronostic visuel de ces patients aurait été très péjoratif. On a donc observé un taux de survie sans progression sans différence entre les deux groupes mais chez des patients qui, sans le traitement par biothérapies, présentaient un risque majeur de cécité.

De plus, il s'agit ici de la comparaison de deux stratégies thérapeutiques utilisées dans le traitement des uvéites, et non pas de la comparaison avec un traitement placebo. De ce fait, 
si une différence existe entre les deux traitements, un effectif plus important peut être nécessaire pour la mettre en évidence. D'un point de vue physiopathologique, si les biothérapies vont cibler avec précision une cytokine, les traitements immunosuppresseurs conventionnels vont avoir un rôle dépresseur immunitaire certain, en ciblant les différents acteurs du système immunitaire, en particulier le lymphocyte T. Comme nous l'avons précisé en introduction, le mycophénolate mofétil est un inhibiteur de la voie de synthèse de novo des bases puriques, voie privilégiée au niveau du lymphocyte $\mathrm{T}$ (46). L'action cytotoxique de l'azathioprine prédomine sur les lymphocytes T. Ainsi, les immunosuppresseurs conventionnels vont limiter l'activation du système immunitaire, de manière moins ciblée certes que les biothérapies, mais à travers des voies de signalisation plus vastes et des acteurs cellulaires plus variés, expliquant leur efficacité.

Les données physiopathologiques issues des modèles murins nous permettent d'émettre des hypothèses sur les mécanismes physiopathologiques des rechutes et de l'échappement thérapeutique (40). Dans les formes récurrentes d'uvéites expérimentales auto-immunes, il a été mis en évidence un défaut de lymphocytes T producteurs à la fois d'IL-17 et d'IL-10, jouant le rôle d'une population régulatrice, et non pas uniquement un rôle pro-inflammatoire comme initialement décrit. Les lymphocytes Th1 semblent impliqués dans l'initiation de la maladie, alors que les lymphocytes Th17 pourraient jouer un rôle plus tardif, montrant ainsi que les cellules impliquées dans la maladie vont évoluer au cours du temps. La plasticité lymphocytaire, influencée par l'environnement cytokinique, peut expliquer le risque de rechute et la variabilité interindividuelle de réponse au traitement.

Notre analyse en sous-groupes comparant les patients recevant une biothérapie en première intention $(n=11)$ versus les patients recevant un immunosuppresseur conventionnel en première intention $(n=39)$, n'a pas mis en évidence de différence significative concernant le taux et le délai médian de survenue du critère de jugement principal, sous réserve du faible effectif. De plus, dans l'étude prospective VISUAL I (52), l'efficacité de l'adalimumab, concernant le taux et le délai de rechute, n'était pas statistiquement supérieure à celle du placebo dans le sous-groupe de patients recevant un immunosuppresseur conventionnel. De futures études prospectives randomisées seront nécessaires pour comparer ces deux stratégies.

Quinze patients (41\%) dans le groupe biothérapie et 19 patients (49\%) dans le groupe immunosuppresseur conventionnel ont présenté une rechute $(\mathrm{p}=0,7)$. Le critère définissant la rechute le plus fréquemment retrouvé dans les deux groupes était l'absence de correction ou l'apparition d'un œdème maculaire cystoïde $(\mathrm{p}=0,74)$. Le délai médian de rechute était allongé dans les deux groupes (29 mois et 41 mois respectivement), comparé au délai de survenue du 
critère de jugement principal, mais sans différence significative entre les deux stratégies. Notre étude a montré une gestion de la rechute similaire dans les deux groupes. Un changement de traitement était plus fréquemment proposé dans le groupe biothérapie $(60 \%)$ contre $47 \%$ dans le groupe immunosuppresseur conventionnel $(\mathrm{p}=0,47)$. En revanche, on a pu observer une tendance à un recours plus fréquent à la corticothérapie sous toute ces formes (implant, injection sous-conjonctivale, majoration de la posologie per os) dans le groupe immunosuppresseur $(\mathrm{p}=0,06)$. Ce résultat ne peut pas être expliqué par une différence de temporalité de la rechute, puisque, parmi les 9 patients ayant bénéficié d'une augmentation de la corticothérapie dans le groupe immunosuppresseur, 7 ont présenté leur rechute après les années 2013, donc bien après le début d'utilisation des biothérapies. Une des hypothèses explicatives est que, les patients traités par biothérapies ayant eu une exposition à la corticothérapie plus longue que les patients sous immunosuppresseurs conventionnels, le prescripteur ait privilégié un changement de thérapeutique plutôt qu'une majoration de la corticothérapie.

Nous avons mis en évidence une correction de l'œdème maculaire sous traitement dans $50 \%$ des cas dans les deux groupes $(\mathrm{p}>0,99)$. L'efficacité tant des biothérapies que des immunosuppresseurs conventionnels dans l'amélioration de l'œdème maculaire a déjà été montrée dans la littérature $(65,81)$. Schaap-Fogler et al. ont comparé les deux stratégies thérapeutiques et n'ont pas mis en évidence de différence significative après 12 mois de traitement en termes de réduction de l'épaisseur maculaire centrale (88). Il est intéressant de noter que, dans notre étude, parmi les 36 patients traités par biothérapies, 8 patients ont bénéficié de ce traitement devant un échec des immunosuppresseurs conventionnels pour contrôler l'œdème maculaire. Parmi ces 8 patients, 3 patients ont eu une correction de l'œdème maculaire sous biothérapies. A nouveau, soulignons ici que, certains patients n'ayant pas répondu au traitement immunosuppresseur conventionnel ont présenté une amélioration d'un paramètre ophtalmologique sous biothérapies. Cependant, dans notre étude, le traitement par anti-TNF alpha n'est pas apparu comme supérieur aux immunosuppresseurs conventionnels dans la correction de l'œdème maculaire. Il s'agit d'un axe majeur d'orientation des nouvelles thérapeutiques devant le pronostic visuel péjoratif incontestable en cas d'apparition d'un œdème maculaire. Ainsi, si les anti-TNF alpha, à partir des données des études VISUAL, semblent limiter le développement d'un œdème maculaire cystoïde sous traitement, un nouvel arsenal thérapeutique apparaît nécessaire pour contrôler un œdème maculaire préexistant. Ici prend tout l'intérêt du tocilizimab, qui va non seulement avoir une action anti-inflammatoire, mais également une action inhibitrice de la sécrétion de VEGF (89), impliquée dans la génération 
des œdèmes maculaires cystoïdes (90). Une étude prospective ouverte a montré l'efficacité du tocilizumab dans le contrôle de l'œdème maculaire (57). Malgré le faible nombre de patients traités par tocilizumab dans notre travail, il est intéressant de relever que ces deux patients ont présenté une résolution de l'œdème maculaire sous traitement.

Notre étude n'a pas mis en évidence de différence significative entre les deux groupes concernant l'amélioration de l'acuité visuelle. Il reste intéressant de relever que $31 \%$ des patients traités par biothérapies ont présenté une amélioration de l'acuité visuelle à 12 mois de traitement, contre seuls $7 \%$ des patients traités par immunosuppresseurs conventionnels $(\mathrm{p}=0,37)$.

Comment expliquer l'absence de différence en termes d'évolution de l'acuité visuelle? A nouveau, les patients sous biothérapies étaient des patients plus sévères, exposés à un risque majeur de cécité devant l'échec des thérapeutiques antérieures. Bien que ce paramètre lors de l'initiation du traitement n'ait pas été différent entre les deux groupes, l'acuité visuelle de l'œil le plus atteint était plus sévère dans le groupe biothérapie $(0,4 \operatorname{LogMAR})$ que dans le groupe immunosuppresseur conventionnel $(0,3 \operatorname{LogMAR})(p=0,63)$. Cette différence, associée à la sévérité des patients traités par biothérapies, peut expliquer, en partie, l'absence de différence entre les deux groupes. Enfin, une baisse d'acuité visuelle peut être la conséquence de différents évènements : majoration de l'inflammation antérieure ou vitréenne, apparition d'un œdème maculaire, complication hémorragique... Il est possible que l'absence de mise en évidence de différence sur l'acuité visuelle soit le reflet d'un contrôle non homogène de ces paramètres ophtalmologiques par les deux stratégies thérapeutiques.

De manière intéressante, notre étude a montré une efficacité plus rapide des biothérapies dans le contrôle de l'inflammation oculaire, avec $92 \%$ des patients présentant un état de quiescence oculaire après 6 mois de traitement, contre $68 \%$ dans le groupe immunosuppresseur conventionnel $(p=0,046)$. La différence entre les deux groupes n'était plus significative après 12 mois de traitement. L'efficacité des biothérapies dans le contrôle de l'inflammation oculaire a déjà été montrée, notamment grâce à l'étude VISUAL I (52). Notre étude a mis en évidence une supériorité des biothérapies par rapport aux immunosuppresseurs conventionnels dans le contrôle rapide de l'inflammation oculaire. A travers ce résultat, les biothérapies apparaissent comme la stratégie de première intention en cas d'atteinte inflammatoire majeure, responsable d'un retentissement sur la fonction visuelle. 
Cependant, l'ensemble des patients ne vont pas être contrôlés sous biothérapies et certains patients vont présenter une inflammation oculaire persistante malgré ces traitements. Au début de l'étude VISUAL III (73), malgré un traitement antérieur par adalimumab, 15,5\% des patients n'ayant pas souffert d'une rechute lors des études parents, ne présentaient pas un état de quiescence oculaire. Dans le sous-groupe d'uvéite active chez les patients ayant déjà rechuté au cours des études VISUAL I et II, l'état de quiescence était obtenu chez seulement $60 \%$ de ces malades à la fin de l'étude VISUAL III. Ainsi, certains patients, indépendamment de la durée de traitement, n'obtiendront pas un état de quiescence inflammatoire malgré le traitement par anti-TNF alpha. Chez ces malades, pourrait se discuter la réalisation d'une ponction de chambre antérieure pour étudier l'environnement cytokinique et adapter la stratégie thérapeutique au patient.

Une décroissance significative de la corticothérapie sous traitement a été observée, sans différence statistique entre les deux groupes. Comment expliquer ce résultat? Indépendamment du traitement d'épargne cortisonique, une décroissance lente de la corticothérapie semble indispensable pour contrôler l'inflammation oculaire. L'étude VISUAL I retrouvait un taux de rechute élevé dans le groupe adalimumab et un délai de rechute de l'ordre de quelques semaines, en partie secondaire, comme l'ont reconnu les auteurs, à leur schéma de décroissance très rapide de la corticothérapie (52). Les effets indésirables de la corticothérapie prolongée sont connus et ont été à nouveau soulignés grâce à l'analyse post-hoc des études VISUAL I et II, avec une majoration de 1,5 du nombre d'effets indésirables pour une augmentation de $10 \mathrm{mg}$ de prednisone (91). Dans ce contexte, les futures études randomisées prospectives devront élaborer un schéma de décroissance de la corticothérapie, alliant une diminution lentement progressive pour favoriser le contrôle de la maladie, tout en atteignant rapidement des posologies $<10 \mathrm{mg} /$ jour de prednisone pour limiter les complications secondaires à une corticothérapie prolongée. Le protocole choisi dans l'essai thérapeutique randomisé prospectif RUBI (ClinicalTrial.gov NCT02929251), comparant l'efficacité et la tolérance de l'adalimumab, le tocilizumab et l'anakinra dans les uvéites, semble réunir ces deux impératifs : posologie à $0,3 \mathrm{mg} / \mathrm{kg}$ à partir de la $8^{\text {ème }}$ semaine de traitement, à $0,2 \mathrm{mg} / \mathrm{kg}$ à partir de la $12^{\text {ème }}$ semaine et un objectif $\leq 0,1 \mathrm{mg} / \mathrm{kg}$ à partir de la $16^{\text {ème }}$ semaine.

De façon intéressante, nous avons montré que, chez les patients ayant rechuté sous biothérapies, antérieurement traités par immunosuppresseurs conventionnels $(\mathrm{n}=10)$, la posologie médiane de corticoïdes lors de la rechute sous biothérapies était statistiquement moins élevée que lors de la rechute sous immunosuppresseurs pour un même patient 
$(\mathrm{p}=0,001)$. Nous n'avons pas mis en évidence de différence entre les deux stratégies thérapeutiques concernant l'épargne cortisonique. En revanche, à l'échelle individuelle, les biothérapies semblent apparaître comme un traitement d'épargne cortisonique supérieur. On peut alors émettre l'hypothèse qu'en cas de cortico-dépendance sous immunosuppresseurs conventionnels, un relais par biothérapies devrait assurer une accélération de la baisse de la corticothérapie.

Cinq patients $(14 \%)$ traités par biothérapies et 4 patients $(10 \%)$ traités par immunosuppresseurs conventionnels ont présenté un effet indésirable grave ayant nécessité d'interrompre le traitement $(\mathrm{p}=0,73)$. Dix-huit patients du groupe biothérapie $(50 \%)$ et 15 patients du groupe immunosuppresseur conventionnel (38\%) ont présenté au moins un effet indésirable au cours du suivi $(\mathrm{p}=0,36)$. Le profil de tolérance, notamment sur les plans infectieux et digestif, ne semble pas différer entre les deux stratégies thérapeutiques. Il y avait une tendance à davantage d'infections dans le groupe traité par biothérapies $(p=0,09)$, majoritairement des infections broncho-pulmonaires virales (8 patients), mais il n'y a eu aucune infection sévère. Dans le groupe immunosuppresseur conventionnel, les patients ayant présenté une infection étaient moins nombreux, mais les infections étaient plus sévères, bien qu'aucune n'ait nécessité d'hospitalisation : une pneumocystose, deux bronchites bactériennes et un abcès dentaire.

Une fois le risque infectieux écarté, toute la gravité dans le maniement de ces thérapeutiques repose sur le risque néoplasique. A partir des données issues de la cohorte SITE, les auteurs n'ont pas mis en évidence d'augmentation du risque néoplasique (solide et hémopathie) sous méthotrexate, mycophénolate mofétil ou azathioprine (92). En revanche, un risque augmenté de cancer était relevé sous anti-TNF alpha. Ce risque a été confirmé dans les études de cohorte de plus grand effectif, chez des patients traités par anti-TNF alpha pour une polyarthrite rhumatoïde (93), où un risque de cancer cutané augmenté a été identifié. De ce fait, le maniement de ces thérapeutiques nécessite une discussion accrue de la balance bénéfice/risque en regard du risque néoplasique.

Une des questions thérapeutiques persistante est celle du choix du traitement pour chaque patient, en fonction de son diagnostic, son histoire thérapeutique et ses comorbidités. Nous n'avons pas mis en évidence de facteurs associés à la prescription de biothérapie. Maleki et al. (75) n'avaient pas retrouvé de facteurs de bonne réponse à l'infliximab dans une population d'uvéite intermédiaire. L'équipe de Fabiani (66) n'avait pas mis en exergue de facteurs prédictifs 
de réponse. Vallet et al. (67) avaient identifié le diagnostic de maladie de Behçet et le nombre de poussées antérieures à l'introduction du traitement $>5$ comme facteurs associés à une réponse complète aux anti-TNF alpha.

L'action bénéfique des anti-TNF alpha dans la maladie de Behçet n'est plus à décrire. Ainsi, dans une étude américaine, 79,5\% des ophtalmologistes spécialisés dans les uvéites interrogés recommandaient la prescription d'une biothérapie en première intention lors du diagnostic d'une maladie de Behçet avec uvéite bilatérale, devant la prescription d'immunosuppresseur conventionnel (7,7\%), indépendamment des limites économiques (94). De plus, une revue de la littérature récente semble souligner une supériorité de l'infliximab versus l'azathioprine ou le méthotrexate, concernant l'amélioration de l'acuité visuelle et du nombre de rechutes dans cette pathologie (95). En regard de ces éléments, un traitement par anti-TNF alpha doit être proposé en première intention, devant les immunosuppresseurs conventionnels, au cours de la maladie de Behçet. Aux vues des recommandations de l'EULAR (19), l'infliximab serait à privilégier. Une étude de 2019, réalisée en ouvert, à comparer l'efficacité de l'adalimumab et de l'infliximab au cours de la maladie de Behçet (96). Pendant l'année de suivi, les auteurs n'ont pas montré de différence entre les deux groupes en termes d'efficacité, de taux de rechute, d'arrêt du traitement pour inefficacité ou d'effets secondaires graves. En revanche, l'acuité visuelle, après un an de traitement, était significativement plus élevée dans le groupe adalimumab. La limite dans l'interprétation de ce résultat est que les auteurs n'ont pas utilisé l'unité du LogMAR pour effectuer les comparaisons et qu'ils ont comparé des chiffres médians d'acuité visuelle, sans définition préalable du critère d'amélioration. Dans ce contexte, de futures études prospectives devront être menées pour pouvoir définitivement conclure.

Concernant la maladie de birdshot, dans cette même étude américaine (94), 76,9 \% des ophtalmologistes interrogés, recommandaient la prescription d'immunosuppresseur conventionnel en première intention lors du diagnostic d'une maladie de birdshot bilatérale, contre seulement 10,3\% recommandant une biothérapie. L'étude VISUAL I ne retrouvait pas de supériorité de l'adalimumab versus placebo dans le sous-groupe de maladie de birdshot (52). De même, dans notre étude, le nombre de patients ayant atteint le critère de jugement principal était plus élevé chez les patients traités par biothérapies que chez les patients traités par immunosuppresseurs conventionnels ( $89 \%$ versus $60 \%$ ). En première intention, un traitement par immunosuppresseurs conventionnels lors de la prise en charge d'une maladie de birdshot est à privilégier, en premier choix le mycophénolate mofétil (64). 
Peu d'études se sont centrées sur les uvéites idiopathiques. Notre étude montre pourtant qu'il s'agit d'uvéites sévères (acuité visuelle altérée), dont le délai médian de survenue du critère de jugement principal était le plus court. Dans l'étude VISUAL I (52), l'étude de sous-groupe portant sur les uvéites idiopathiques a montré une différence significative en faveur de l'adalimumab. Dans notre étude, $82 \%$ des patients recevant un immunosuppresseur conventionnel ont atteint le critère de jugement principal contre $67 \%$ des patients traités par biothérapies. Devant nos résultats, épaulés par les analyses de l'étude VISUAL I, il semble pertinent de proposer rapidement un recours aux biothérapies dans la prise en charge des uvéites idiopathiques, en deuxième intention après un premier échec sous immunosuppresseur conventionnel, voire en première intention en cas d'inflammation oculaire majeure.

Concernant la sarcoïdose, la variabilité des atteintes oculaires et la fréquence des atteintes systémiques rendent difficile une recommandation générale. Nous avons retrouvé que $56 \%$ des patients traités par immunosuppresseurs conventionnels ont présenté le critère de jugement principal contre $67 \%$ des patients ayant reçu une biothérapie, incitant ainsi à privilégier les immunosuppresseurs conventionnels en première intention. Le méthotrexate (31) et le mycophénolate mofétil (97) ont tous les deux montré leur efficacité dans le contrôle de l'inflammation oculaire au cours de la sarcoïdose. Dans notre étude, aucun patient n'a reçu de mycophénolate mofétil.

Les éléments d'ordre économique vont participer à ce choix de traitement. A partir des études VISUAL I et II, le ratio coût-efficacité incrémentiel (ICER) de l'adalimumab, comparé au groupe placebo (recevant dans $31 \%$ des cas un traitement immunosuppresseur conventionnel), était de $92600 £$ par année de vie ajustée sur la qualité (QALY) pour les uvéites actives et de $318075 £$ pour les uvéites inactives (98). Aux vues de ces résultats, en cas d'uvéite inactive mais cortico-dépendante, les traitements immunosuppresseurs conventionnels seront privilégiés. En revanche, en cas d'uvéite active, en cas de pronostic visuel engagé, un traitement par anti-TNF alpha pourra être discuté en première intention devant un gain en qualité de vie et en degrés de handicap.

La dernière question devant être soulevée dans la réflexion thérapeutique actuelle est celle de l'espacement des injections de traitement ou de la diminution des posologies. Deux patients dans notre étude ont rechuté lors de l'espacement des injections d'infliximab et un patient a rechuté 12 mois après l'arrêt de l'infliximab. Un patient a rechuté lors de la diminution des doses de mycophénolate mofétil et 3 patients ont rechuté plus de 6 mois après l'arrêt de 
l'azathioprine et du méthotrexate. Shakoor et al. (99) ont montré un taux de rechute de $61,1 \%$ après l'arrêt de l'infliximab, avec un délai médian de 20 mois.

La majorité de nos patients ont présenté une rechute sous traitement, sans modification de la fréquence des injections ou de diminution de la posologie. De plus, d'après la littérature, l'arrêt du traitement expose les patients à un fort risque de rechute. Ainsi, l'espacement et/ou la diminution des posologies puis l'arrêt des thérapeutiques, devront être soigneusement discutés chez ces patients, sous couvert d'une évaluation ophtalmologique rapprochée.

\subsection{Forces et limites de l'étude}

Le point fort de notre étude était la rigueur de définition de la rechute, ne reposant non pas sur l'arrêt du traitement, mais sur des critères ophtalmologiques objectifs. A notre connaissance, il s'agit de la première étude rétrospective comparant le risque de récidive et les effets indésirables graves sous immunosuppresseurs conventionnels versus biothérapies dans les uvéites non-infectieuses non-antérieures. Notre étude a le mérite de souligner des problématiques thérapeutiques très actuelles dans le domaine de l'œil inflammatoire, concernant le choix du traitement, les espacements du traitement, les questions d'économie de la santé, et d'y apporter des éléments de réponses.

Notre étude comporte un certain nombre de limites. Tout d'abord, il s'agit d'une étude rétrospective, comportant ses risques propres avec un biais de sélection et un biais de mesure. Pour remédier à ces risques, la sélection des patients a été contrôlée par un ophtalmologiste, afin de s'assurer des diagnostics et des localisations des uvéites. Comme nous l'avons déjà précisé en amont, notre principal biais est qu'il existe une différence dans la comparabilité de nos deux groupes. Nous avons choisi d'exclure les patients en cours de traitement par interféron alpha-2a devant leur faible nombre (7 patients). De plus, ces traitements ne peuvent pas être considérés comme des immunosuppresseurs conventionnels, mais leur mécanisme d'action les rapproche des biothérapies, sans pour autant correspondre aux biothérapies actuelles (anticorps monoclonal ciblant une cible cytokinique). Nous avons également fait ce choix pour nous approcher des critères d'inclusion des études VISUAL, où les patients traités de façon concomitante par interférons alpha-2a étaient exclus. Cependant, il est certain que ces thérapeutiques devront s'intégrer dans l'arsenal thérapeutique des uvéites. Enfin, nous avons choisi d'inclure dans les critères de rechute, l'absence d'évolution favorable de l'œdème maculaire. Or, l'œdème maculaire est parfois considéré par certains auteurs comme une 
complication de l'uvéite, sans correspondre à un signe d'activité de celle-ci. Les données de la littérature ne sont pas univoques quant à la considération de ce seul paramètre pour intensifier le traitement (45). Cependant, nous avons fait ce choix pour nous rapprocher des conditions réelles de prescription, puisque la persistance d'un œdème maculaire va entraîner un risque pour le pronostic visuel et donc une sanction thérapeutique. 


\section{Conclusion}

Les uvéites non-infectieuses non-antérieures représentent une cause majeure de cécité dans les pays industrialisés, responsables d'un handicap fonctionnel chez les sujets jeunes. Par conséquent, la gestion de ces uvéites et de leurs complications se révèle, aujourd'hui, comme un nouvel enjeu pour les internistes et les ophtalmologistes. Entité aux multiples facettes, sont réunies sous le terme d'uvéite des étiologies différentes, aux mécanismes physiopathologiques divergents. La prise en charge thérapeutique vise à contrôler rapidement et de manière durable l'inflammation intraoculaire, pour réduire l'apparition de séquelles anatomiques irrémédiables, tout en limitant les effets indésirables des thérapeutiques, notamment ceux d'une corticothérapie prolongée. La cortico-résistance et la cortico-dépendance apparaissent comme des éléments nécessitants, à eux seuls, une intensification thérapeutique. La question actuelle n'est pas de discuter l'efficacité des biothérapies dans le traitement des uvéites, les études VISUAL l'ayant largement démontrée. La principale interrogation réside aujourd'hui dans la place hiérarchique de chaque traitement dans la stratégie thérapeutique, et notamment celle des immunosuppresseurs conventionnels devant l'avènement des biothérapies.

Notre étude n'a pas mis en évidence de différence significative entre les patients traités par immunosuppresseurs conventionnels ou biothérapies, en termes de survie sans progression, d'amélioration de l'acuité visuelle ou de résolution de l'œdème maculaire cystoïde. En revanche, notre étude a montré un contrôle de l'inflammation intraoculaire plus rapide dans le groupe biothérapie. Concernant l'épargne cortisonique, notre étude a souligné que, pour un même patient, la cortico-dépendance au moment de la rechute était moins élevée lors du traitement par biothérapies que lors du traitement par immunosuppresseurs conventionnels. Nos résultats mettent en exergue que les immunosuppresseurs conventionnels conservent une place réelle dans la stratégie thérapeutique actuelle des uvéites inflammatoires, devant leur efficacité, leur coût maîtrisé et leur tolérance. Ainsi, ces thérapeutiques doivent rester les traitements de premiers choix en cas d'uvéite active insuffisamment contrôlée par une corticothérapie ou en cas de cortico-dépendance. Les biothérapies semblent apparaître comme la stratégie de premiers recours en cas d'inflammation sévère menaçante, devant leur rapidité de contrôle de celle-ci, notamment lors de la maladie de Behçet. De plus, en cas de cortico-dépendance malgré un traitement immunosuppresseur conventionnel, les biothérapies se révèlent plus efficaces pour assurer une épargne cortisonique. Le tocilizumab semble apparaître comme le traitement de choix dans la réduction de l'œdème maculaire cystoïde. 
Nos résultats montrent également que les uvéites restent des pathologies à fort risque de rechute, indépendamment du traitement initial employé, et la vie thérapeutique de chaque patient sera égrenée de plusieurs lignes thérapeutiques, jouant sur les différentes voies de contrôle du système immunitaire. La diminution de la pression thérapeutique sur ces patients devra être extrêmement prudente, devant un risque de recrudescence inflammatoire prééminent.

Les études prospectives à venir devront non seulement comparer ces deux stratégies thérapeutiques en première intention mais également s'intéresser, indépendamment, à chacune des pathologies responsables d'uvéites, puisqu'il apparaît que la physiopathologie et donc la réponse au traitement sont différentes en fonction du diagnostic étiologique. Enfin, ces futures études devront évaluer la durée du traitement, et la pertinence d'éventuel schéma de décroissance des immunosuppresseurs.

A l'heure de la médecine centrée sur le patient, les stratégies thérapeutiques futures devront être orientées non seulement par le diagnostic étiologique, les comorbidités du patient et son degré de handicap, mais aussi par le diagnostic immunologique, à travers l'analyse de l'environnement cytokinique oculaire, propre à chaque malade. 
Annexe 1 : Liste des codages CIM10 ayant permis l'extraction des données des patients suivis en médecine interne.

H20.0 Iridocyclite aiguë et subaiguë

H20.1 Iridocyclite chronique

H20.2 Iridocyclite due aux lentilles de contact

H20.8 Autres iridocyclites

H20.9 Iridocyclite

H22.0 Iridocyclite pendant maladie infectieuse et parasitaire et A18.5 Tuberculose de l'œil

H22.1 Iridocyclite pendant autres maladies et D86.8 Sarcoïdose de localisations autres et associées

H22.1 Iridocyclite pendant autres maladies et M45 Spondylarthrite ankylosante

H30.0 Chorioretinite en foyer

H30.1 Chorioretininite disséminée

H30.2 Cyclite postérieure

H30.8 Autres chorioretinites

H30.9 Chorioretinite

H44.1 Autres endophtalmies 


\section{Annexe 2 : Définition de l'acuité visuelle et différentes échelles.}

\section{Définition de l'acuité visuelle (62) :}

L'acuité visuelle est quantifiée par le pouvoir séparateur de l'œil, c'est-à-dire par la taille angulaire minimale permettant de distinguer un objet. L'angle MAR ou AMR (angle minimum de résolution) est l'angle visuel que doivent sous-tendre deux sources ponctuelles pour être résolues sur la rétine de l'œil. Connaître l'angle MAR est une façon de quantifier le pouvoir de résolution de l'œil. La majorité des sujets jeunes est capable de distinguer 2 objets séparés par un angle d'une minute d'arc soit $1 / 60^{\circ}$ de degré. Cette valeur de 1 minute d'arc a été retenue comme valeur de normalité. L'acuité visuelle est égale à l'inverse de l'angle MAR : un angle de résolution minimum de 1 minute correspond à une acuité de 10/10.

Les différentes échelles d'acuité visuelle (63) :

L'échelle de Monoyer, est la plus utilisée en France en pratique clinique. Les optotypes sont lus à cinq mètres et sont représentés par des lettres majuscules de taille croissante, comprenant 10 à 12 valeurs d'acuité, allant de 0,1 à 1,0 ou 1,2. Cette échelle privilégie la mesure des bonnes acuités visuelles au détriment des basses acuités visuelles, en raison de sa progression discontinue. En effet, elle a comme inconvénient majeur sa progression arithmétique, qui fait que la différence d'angle apparent est beaucoup plus grande entre $1 / 10$ et $2 / 10$ qu'entre $9 / 10$ et

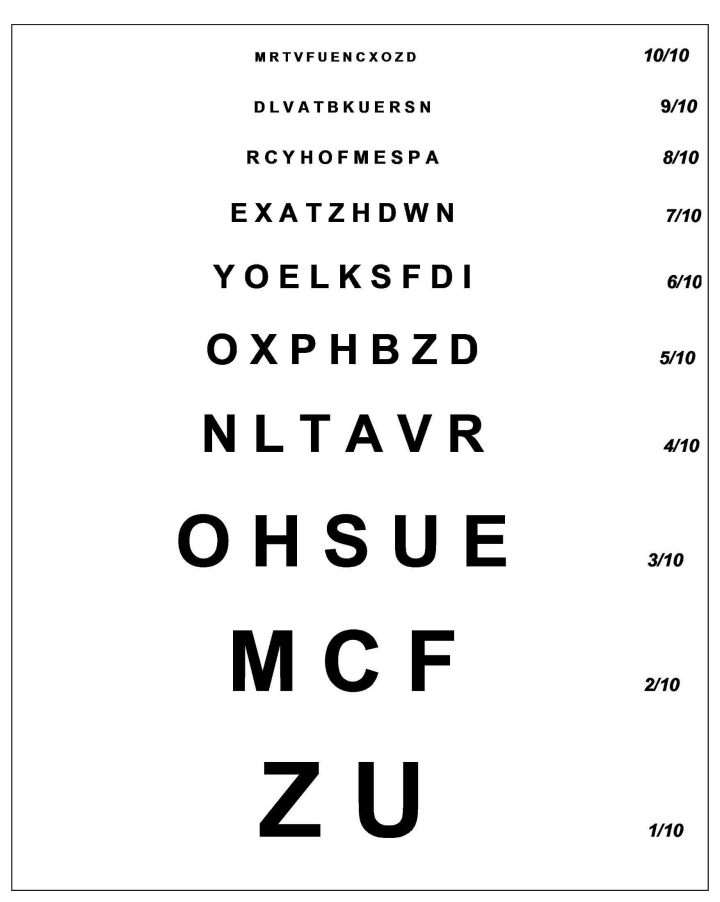
$10 / 10$.

Cet inconvénient n'existe pas lorsque l'on utilise des échelles logarithmiques. L'intervalle entre les lignes d'une échelle d'acuité logarithmique est identique sur toute l'étendue de l'échelle. L'échelle Early Treatment Diabetic Retinopathy Study ou ETDRS est une échelle logarithmique. L'échelle R sert à mesurer la réfraction du patient, l'échelle 1 à tester l'œil droit et l'échelle 2 l'œil gauche. Chaque échelle présente 14 lignes de 5 lettres chacune. Les lettres 
de chaque ligne sont plus hautes que celles de la ligne inférieure d'un coefficient constant. Une baisse d'acuité visuelle de trois lignes équivaut à un doublement de l'angle visuel, quelle que soit l'acuité visuelle d'origine.

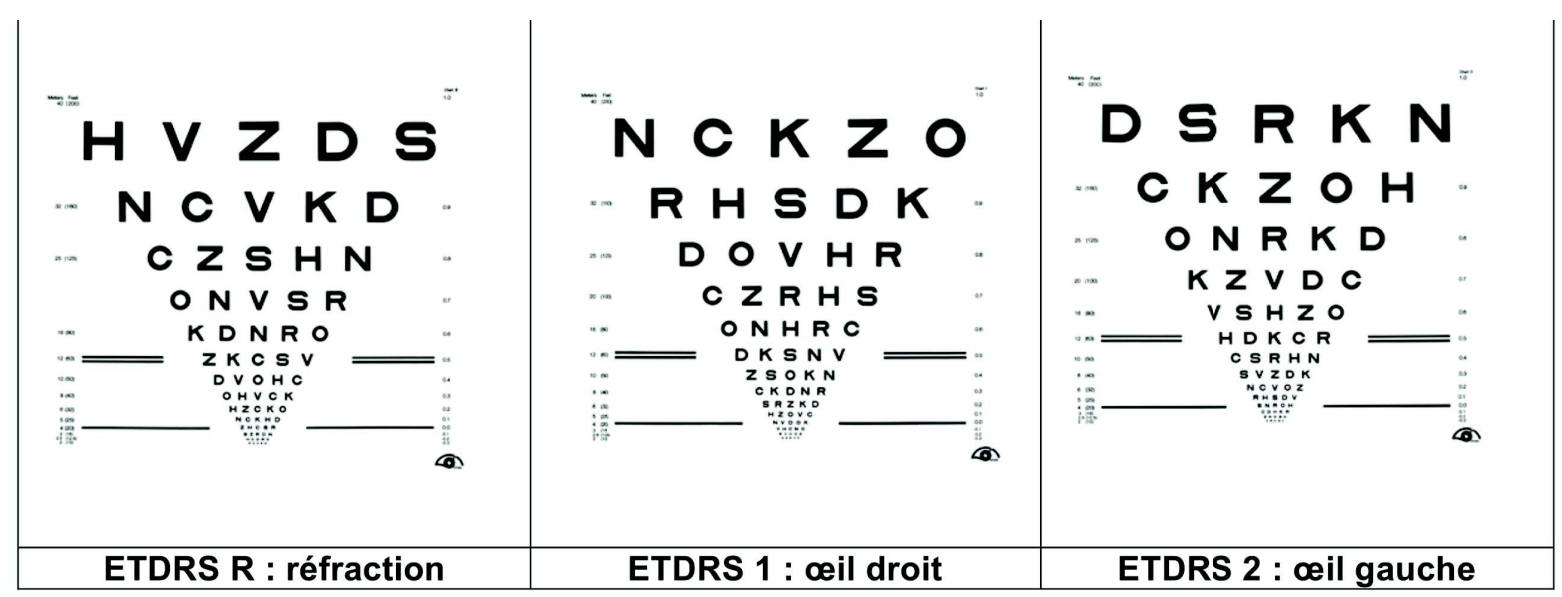

Le LogMAR est une unité qui permet de quantifier l'acuité visuelle de manière à pouvoir effectuer des calculs statistiques. Un même gain de lignes d'acuité visuelle (ce gain est le reflet d'un certain pourcentage de réduction du MAR) est converti en un nombre constant de $\log$ MAR, indépendamment de la valeur du MAR initial. 
Annexe 3 : E-poster présenté en section orale commentée le vendredi 30 aout 2019 au Congrès Européen de Médecine Interne à Lisbonne.
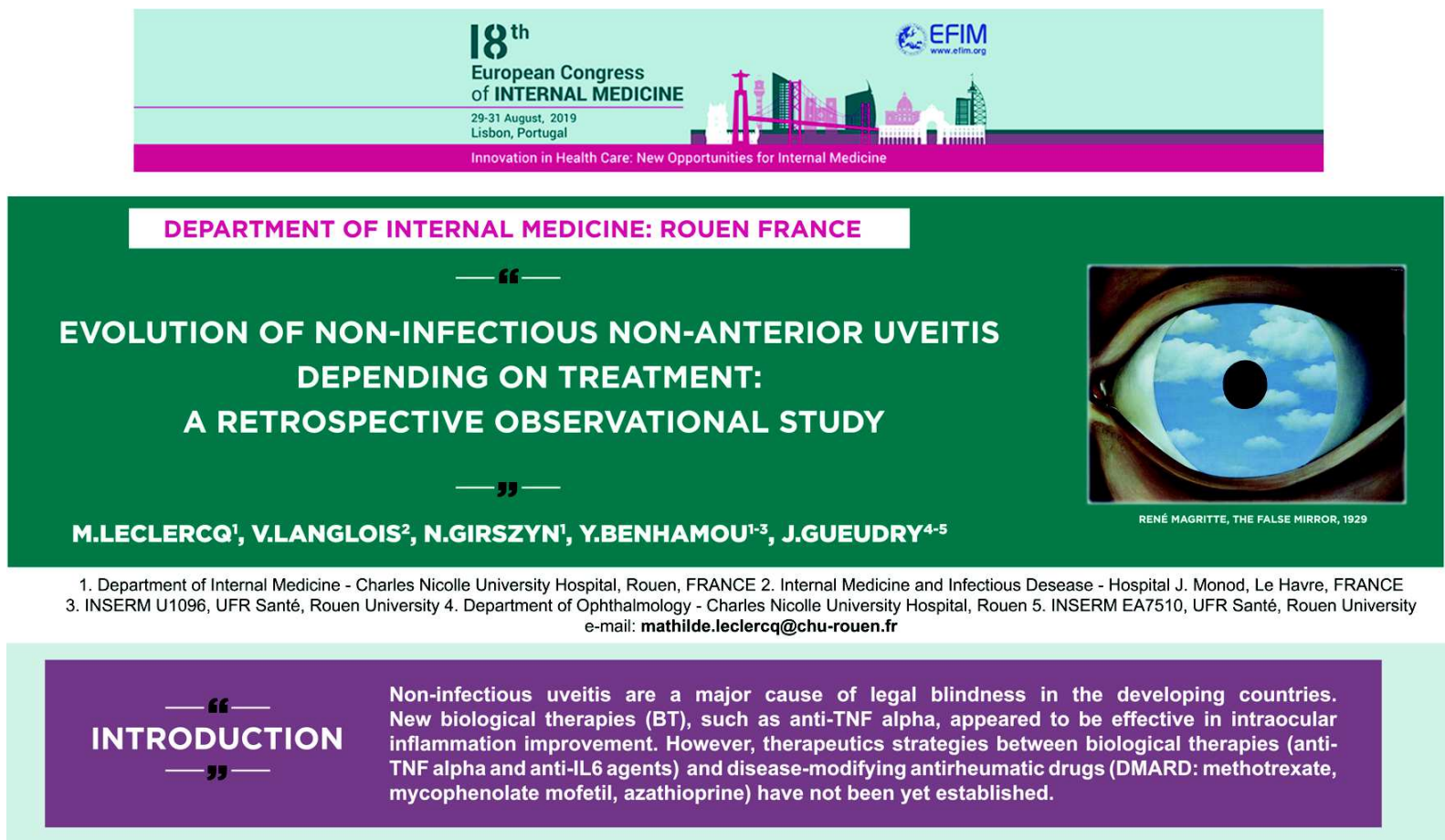
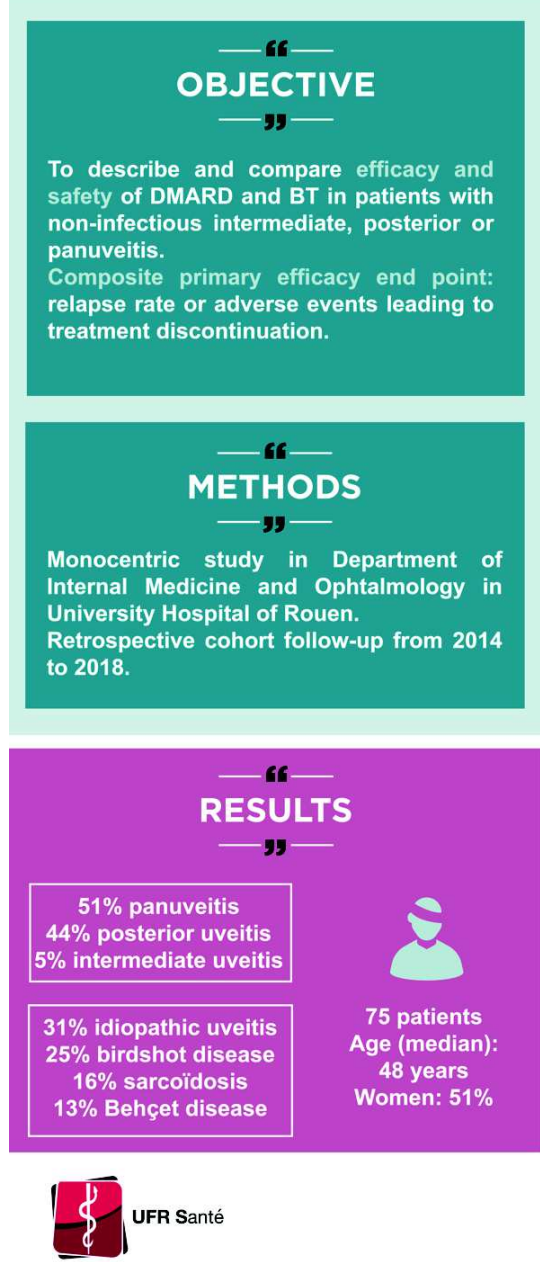

DMARD are as efficient as BT for relapse rate, macular edema improvement and corticosteroid sparing in non-infectious nonanterior uveitis. DMARD must still be used in uveitis treatment.
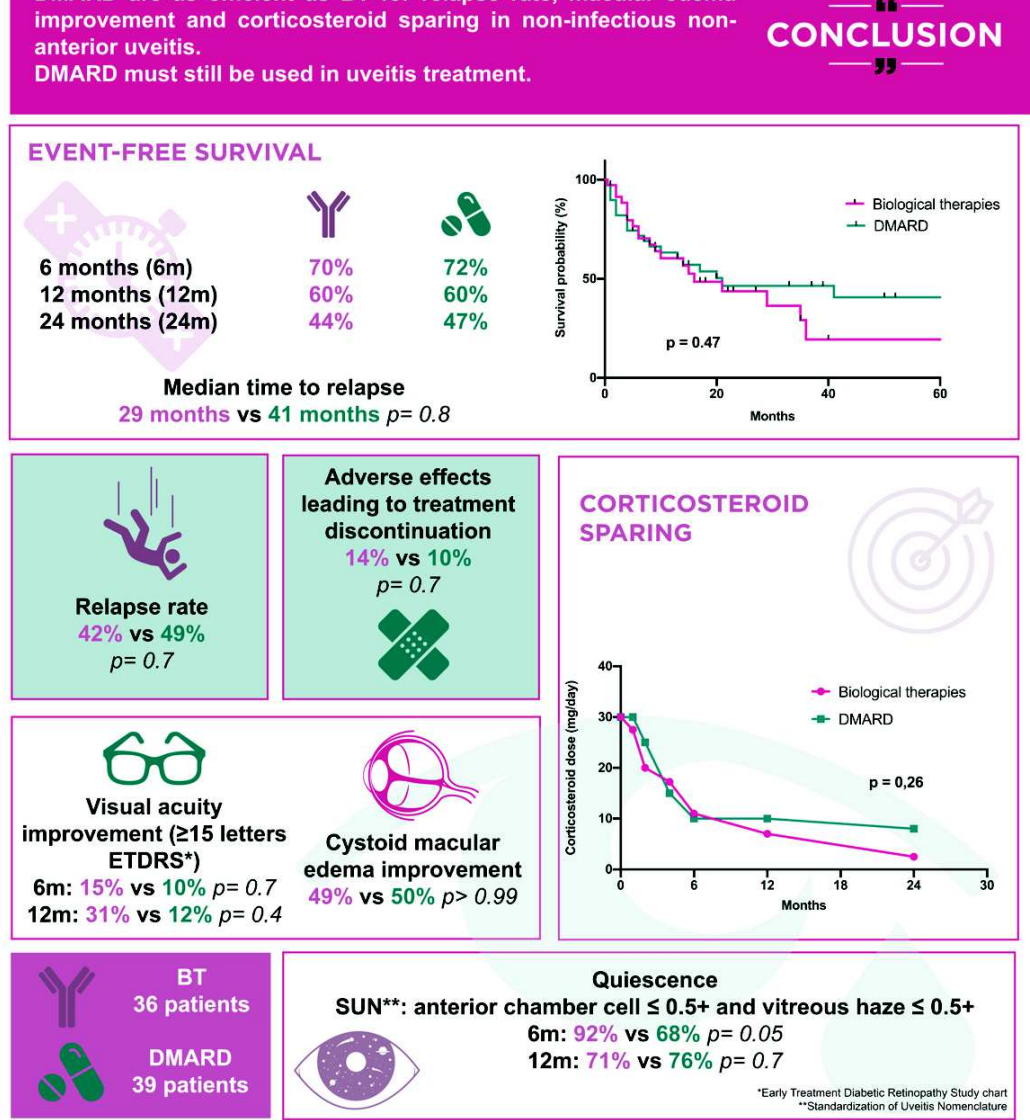

Cystoid macular edema improvement $49 \%$ vs $50 \% p>0.99$

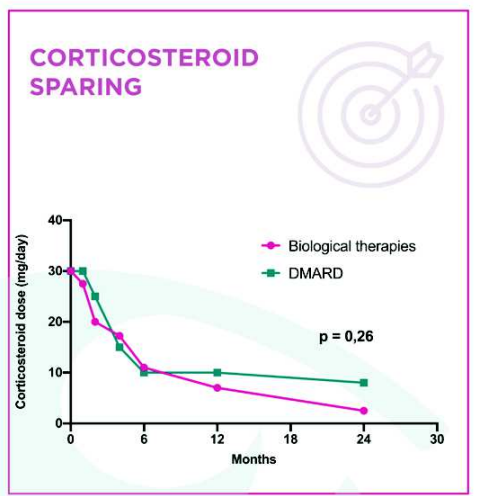

\section{Quiescence}

SUN**: anterior chamber cell $\leq 0.5+$ and vitreous haze $\leq 0.5+$ $6 \mathrm{~m}: 92 \%$ vs $68 \% p=0.05$ 12m: $71 \%$ vs $76 \% p=0.7$

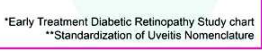




\section{Bibliographie}

1. Bodaghi B, LeHoang P. Uvéite. Atlas en ophtalmologie. Elsevier Masson. 2017.

2. Bloch-Michel E, Nussenblatt RB. International Uveitis Study Group recommendations for the evaluation of intraocular inflammatory disease. Am J Ophthalmol. 1987;103(2):234-5.

3. Jabs DA, Nussenblatt RB, Rosenbaum JT, Standardization of Uveitis Nomenclature (SUN) Working Group. Standardization of uveitis nomenclature for reporting clinical data. Results of the First International Workshop. Am J Ophthalmol. 2005;140(3):509-16.

4. Polycopié national du collège des ophtalmologistes universitaires de France. Elsevier Masson. 2010.

5. Prete M, Dammacco R, Fatone MC, Racanelli V. Autoimmune uveitis: clinical, pathogenetic, and therapeutic features. Clin Exp Med. 2016;16(2):125-36.

6. Sève $\mathrm{P}$, Kodjikian L, Adélaïde L, Jamilloux Y. Uveitis in adults: What do rheumatologists need to know? Joint Bone Spine. 2015;82(5):308-14.

7. Tsirouki T, Dastiridou A, Symeonidis C, Tounakaki O, Brazitikou I, Kalogeropoulos C, et al. A Focus on the Epidemiology of Uveitis. Ocul Immunol Inflamm. 2018;26(1):2-16.

8. Sève P, Bodaghi B, Trad S, Sellam J, Bellocq D, Bielefeld P, et al. Uveitis: Diagnostic work-up. Recommendations from an expert committee. Rev Med Interne. 2018;39(9):676-86.

9. Barisani-Asenbauer T, Maca SM, Mejdoubi L, Emminger W, Machold K, Auer H. Uveitis- a rare disease often associated with systemic diseases and infections- a systematic review of 2619 patients. Orphanet J Rare Dis. 2012;7:57.

10. Bodaghi B, Cassoux N, Wechsler B, Hannouche D, Fardeau C, Papo T, et al. Chronic severe uveitis: etiology and visual outcome in 927 patients from a single center. Medicine (Baltimore). 2001;80(4):263-270.

11. Nussenblatt RB, Palestine AG, Chan CC, Roberge F. Standardization of vitreal inflammatory activity in intermediate and posterior uveitis. Ophthalmology. 1985;92(4):467-71.

12. Thorne JE, Suhler E, Skup M, Tari S, Macaulay D, Chao J, et al. Prevalence of Noninfectious Uveitis in the United States: A Claims-Based Analysis. JAMA Ophthalmol. 2016;134(11):1237-45.

13. Dick AD, Tundia N, Sorg R, Zhao C, Chao J, Joshi A, et al. Risk of Ocular Complications in Patients with Noninfectious Intermediate Uveitis, Posterior Uveitis, or Panuveitis. Ophthalmology. 2016;123(3):655-62.

14. Kempen JH, Van Natta ML, Altaweel MM, Dunn JP, Jabs DA, Lightman SL, et al. Factors Predicting Visual Acuity Outcome in Intermediate, Posterior, and Panuveitis: The Multicenter Uveitis Steroid Treatment (MUST) Trial. Am J Ophthalmol. 2015;160(6):1133- 1141 . 
15. de Parisot A, Kodjikian L, Errera M-H, Sedira N, Heron E, Pérard L, et al. Randomized Controlled Trial Evaluating a Standardized Strategy for Uveitis Etiologic Diagnosis (ULISSE). Am J Ophthalmol. 2017;178:176-85.

16. Desbois A-C, Terrada C, Cacoub P, Bodaghi B, Saadoun D. Ocular manifestations in Behçet's disease. Rev Med Interne. 2018;39(9):738-45.

17. Arevalo JF, Lasave AF, Al Jindan MY, Al Sabaani NA, Al-Mahmood AM, Al-Zahrani YA, et al. Uveitis in Behçet Disease in a Tertiary Center Over 25 Years: The KKESH Uveitis Survey Study Group. Am J Ophthalmol. 2015;159(1):177-184.

18. Bazvand F, Zarei M, Ebrahimiadib N, Karkhaneh R, Davoudi S, Soleimanzadeh M, et al. Ocular Manifestations, Conventional Fundus Fluorescein Angiographic Findings, and Relationship Between Angiographic Findings and Visual Acuity in Behçet's Disease. Semin Ophthalmol. 2017;32(6):764-71.

19. Hatemi G, Christensen R, Bang D, Bodaghi B, Celik AF, Fortune F, et al. 2018 update of the EULAR recommendations for the management of Behçet's syndrome. Ann Rheum Dis. 2018;77(6):808-18.

20. Minos E, Barry RJ, Southworth S, Folkard A, Murray PI, Duker JS, et al. Birdshot chorioretinopathy: current knowledge and new concepts in pathophysiology, diagnosis, monitoring and treatment. Orphanet J Rare Dis. 2016;11(1):61.

21. Kiss S, Ahmed M, Letko E, Foster CS. Long-term Follow-up of Patients with Birdshot Retinochoroidopathy Treated with Corticosteroid-Sparing Systemic Immunomodulatory Therapy. Ophthalmology. 2005;112(6):1066-1071.

22. Doycheva D, Jägle H, Zierhut M, Deuter C, Blumenstock G, Schiefer U, et al. Mycophenolic acid in the treatment of birdshot chorioretinopathy: long-term follow-up. $\mathrm{Br} \mathrm{J}$ Ophthalmol. 2015;99(1):87-91.

23. Tomkins-Netzer O, Taylor SRJ, Lightman S. Long-term clinical and anatomic outcome of birdshot chorioretinopathy. JAMA Ophthalmol. 2014;132(1):57-62.

24. Tavallali A, Yannuzzi LA. Idiopathic Multifocal Choroiditis. J Ophthalmic Vis Res. 2016;11(4):429-32.

25. Valeyre D, Prasse A, Nunes H, Uzunhan Y, Brillet P-Y, Müller-Quernheim J. Sarcoidosis. The Lancet. 2014;383(9923):1155-67.

26. Ungprasert P, Wijarnpreecha K, Cheungpasitporn W, Thongprayoon C, Manatsathit W, Kröner PT. Inpatient Prevalence, Expenditures, and Comorbidities of Sarcoidosis: Nationwide Inpatient Sample 2013-2014. Lung. 2019;197(2):165-171.

27. Baughman RP, Teirstein AS, Judson MA, Rossman MD, Yeager H, Bresnitz EA, et al. Clinical characteristics of patients in a case control study of sarcoidosis. Am J Respir Crit Care Med.2001;164(10 Pt 1):1885-9. 
28. Jamilloux Y, Kodjikian L, Broussolle C, Sève P. Sarcoidosis and uveitis. Autoimmun Rev. 2014;13(8):840-9.

29. Mochizuki M, Smith JR, Takase H, Kaburaki T, Acharya NR, Rao NA, et al. Revised criteria of International Workshop on Ocular Sarcoidosis (IWOS) for the diagnosis of ocular sarcoidosis. Br J Ophthalmol. 2019; Epub ahead of print.

30. Sève P, Kodjikian L, Jamilloux Y. Ocular sarcoidosis: What the internist should know?. Rev Med Interne. 2018;39(9):728-37.

31. Baughman RP, Lower EE, Ingledue $\mathrm{R}$, Kaufman AH. Management of ocular sarcoidosis. Sarcoidosis Vasc Diffuse Lung Dis Off J WASOG. 2012;29(1):26-33.

32. Bhat P, Cervantes-Castañeda RA, Doctor PP, Anzaar F, Foster CS. Mycophenolate mofetil therapy for sarcoidosis-associated uveitis. Ocul Immunol Inflamm. 2009;17(3):185-90.

33. Riancho-Zarrabeitia L, Calvo-Río V, Blanco R, Mesquida M, Adan AM, Herreras JM, et al. Anti-TNF- $\alpha$ therapy in refractory uveitis associated with sarcoidosis: Multicenter study of 17 patients. Semin Arthritis Rheum. 2015;45(3):361-8.

34. Bonnet C, Daudin J-B, Monnet D, Brézin A. Vogt-Koyanagi-Harada disease. J Fr Ophtalmol. 2017;40(6):512-9.

35. Abu El-Asrar AM, Hemachandran S, Al-Mezaine HS, Kangave D, Al-Muammar AM. The outcomes of mycophenolate mofetil therapy combined with systemic corticosteroids in acute uveitis associated with Vogt-Koyanagi-Harada disease. Acta Ophthalmol (Copenh). 2012;90(8):e603-608.

36. Kim SJ, Yu HG. The use of low-dose azathioprine in patients with Vogt-KoyanagiHarada disease. Ocul Immunol Inflamm. 2007;15(5):381-7.

37. Takayama K, Obata H, Takeuchi M. Efficacy of Adalimumab for Chronic VogtKoyanagi-Harada Disease Refractory to Conventional Corticosteroids and Immunosuppressive Therapy and Complicated by Central Serous Chorioretinopathy. Ocul Immunol Inflamm. 2019; Epub ahead of print.

38. Bose T, Diedrichs-Möhring M, Wildner G. Dry eye disease and uveitis: A closer look at immune mechanisms in animal models of two ocular autoimmune diseases. Autoimmun Rev. 2016;15(12):1181-92.

39. Perez VL, Caspi RR. Immune mechanisms in inflammatory and degenerative eye disease. Trends Immunol. 2015;36(6):354-63.

40. Diedrichs-Möhring M, Kaufmann U, Wildner G. The immunopathogenesis of chronic and relapsing autoimmune uveitis - Lessons from experimental rat models. Prog Retin Eye Res. 2018;65:107-26.

41. Weinstein JE, Pepple KL. Cytokines in uveitis. Curr Opin Ophthalmol. 2018;29(3):267-74. 
42. Gery I, Caspi RR. Tolerance Induction in Relation to the Eye. Front Immunol. 2018;9:2304.

43. Diwo E, Sève P, Trad S, Bielefeld P, Sène D, Abad S, et al. Therapeutic strategy for the treatment of non-infectious uveitis proposed by an expert panel. Rev Med Interne. 2018;39(9):687-698.

44. Writing Committee for the Multicenter Uveitis Steroid Treatment (MUST) Trial and Follow-up Study Research Group, Kempen JH, Altaweel MM, Holbrook JT, Sugar EA, Thorne JE, et al. Association Between Long-Lasting Intravitreous Fluocinolone Acetonide Implant vs Systemic Anti-inflammatory Therapy and Visual Acuity at 7 Years Among Patients With Intermediate, Posterior, or Panuveitis. JAMA. 2017;317(19):1993-2005.

45. Dick AD, Rosenbaum JT, Al-Dhibi HA, Belfort R, Brézin AP, Chee SP, et al. Guidance on Noncorticosteroid Systemic Immunomodulatory Therapy in Noninfectious Uveitis: Fundamentals Of Care for UveitiS (FOCUS) Initiative. Ophthalmology. 2018;125(5):757-73.

46. Guillevin L, Meyer O, Hachulla E, Sibilia J. Traité des maladies et syndromes systémiques. Lavoisier. 2015.

47. Kempen JH, Daniel E, Gangaputra S, Dreger K, Jabs DA, Kaçmaz RO, et al. Methods for identifying long-term adverse effects of treatment in patients with eye diseases: the Systemic Immunosuppressive Therapy for Eye Diseases (SITE) Cohort Study. Ophthalmic Epidemiol. 2008;15(1):47-55.

48. Gangaputra S, Newcomb CW, Liesegang TL, Kaçmaz RO, Jabs DA, Levy-Clarke GA, et al. Methotrexate for ocular inflammatory diseases. Ophthalmology. 2009;116(11):2188 - 2198 .

49. Daniel E, Thorne JE, Newcomb CW, Pujari SS, Kaçmaz RO, Levy-Clarke GA, et al. Mycophenolate mofetil for ocular inflammation. Am J Ophthalmol. 2010;149(3):423-432.

50. Pasadhika S, Kempen JH, Newcomb CW, Liesegang TL, Pujari SS, Rosenbaum JT, et al. Azathioprine for ocular inflammatory diseases. Am J Ophthalmol. 2009;148(4):500-509.

51. Galor A, Jabs DA, Leder HA, Kedhar SR, Dunn JP, Peters GB, et al. Comparison of antimetabolite drugs as corticosteroid-sparing therapy for noninfectious ocular inflammation. Ophthalmology. 2008;115(10):1826-32.

52. Jaffe GJ, Dick AD, Brézin AP, Nguyen QD, Thorne JE, Kestelyn P, et al. Adalimumab in Patients with Active Noninfectious Uveitis. N Engl J Med. 2016;375(10):932-43.

53. Nguyen QD, Merrill PT, Jaffe GJ, Dick AD, Kurup SK, Sheppard J, et al. Adalimumab for prevention of uveitic flare in patients with inactive non-infectious uveitis controlled by corticosteroids (VISUAL II): a multicentre, double-masked, randomised, placebo-controlled phase 3 trial. The Lancet. 2016;388(10050):1183-92.

54. Al-Rayes H, Al-Swailem R, Al-Balawi M, Al-Dohayan N, Al-Zaidi S, Tariq M. Safety and efficacy of infliximab therapy in active behcet's uveitis: an open-label trial. Rheumatol Int. 2008;29(1):53-7. 
55. Giardina A, Ferrante A, Ciccia F, Vadalà M, Giardina E, Triolo G. One year study of efficacy and safety of infliximab in the treatment of patients with ocular and neurological Behçet's disease refractory to standard immunosuppressive drugs. Rheumatol Int. 2011;31(1):33-7.

56. Silpa-archa S, Oray M, Preble JM, Foster CS. Outcome of tocilizumab treatment in refractory ocular inflammatory diseases. Acta Ophthalmol (Copenh). 2016;94(6).

57. Sepah YJ, Sadiq MA, Chu DS, Dacey M, Gallemore R, Dayani P, et al. Primary (Month6) Outcomes of the STOP-Uveitis Study: Evaluating the Safety, Tolerability, and Efficacy of Tocilizumab in Patients with Non-Infectious Uveitis. Am J Ophthalmol. 2017; 183:71-80.

58. Kötter I, Günaydin I, Zierhut M, Stübiger N. The use of interferon alpha in Behçet disease: review of the literature. Semin Arthritis Rheum. 2004;33(5):320-35.

59. Gueudry J, Wechsler B, Terrada C, Gendron G, Cassoux N, Fardeau C, et al. Long-term efficacy and safety of low-dose interferon alpha2a therapy in severe uveitis associated with Behçet disease. Am J Ophthalmol. 2008;146(6):837-844.

60. Fardeau C, Simon A, Rodde B, Viscogliosi F, Labalette P, Looten V, et al. Interferonalpha2a and Systemic Corticosteroid in Monotherapy in Chronic Uveitis: Results of the Randomized Controlled BIRDFERON Study. Am J Ophthalmol. 2017;177:182-94.

61. Neri P, Arapi I, Nicolai M, Pirani V, Saitta A, Luchetti MM, et al. Biologic Therapy in Inflammatory Eye Conditions (Ophtalmology): Safety Profile. Curr Drug Saf. 2016;11(1):47-54.

62. O.Touzeau. Calculs de l'acuité visuelle moyenne et de la variation d'acuité visuelle à partir d'une échelle décimale. Journal français d'ophtalmologie. Vol. 26.2003.

63. Vignal-Clermont C, Tilikete C, Miléa D. Chapitre2 - Moyens d'étude de la fonction visuelle. Neuro-Ophtalmologie. Elsevier Masson. 2016.

64. Touhami S, Diwo E, Sève P, Trad S, Bielefeld P, Sène D, et al. Expert opinion on the use of biological therapy in non-infectious uveitis. Expert Opin Biol Ther. 2019;19(5):477-90.

65. Díaz-Llopis M, Salom D, Garcia-de-Vicuña C, Cordero-Coma M, Ortega G, Ortego N, et al. Treatment of Refractory Uveitis with Adalimumab: A Prospective Multicenter Study of 131 Patients. Ophthalmology. 2012;119(8):1575-81.

66. Fabiani C, Vitale A, Emmi G, Bitossi A, Lopalco G, Sota J, et al. Long-term retention rates of adalimumab and infliximab in non-infectious intermediate, posterior, and panuveitis. Clin Rheumatol. 2019;38(1):63-70.

67. Vallet H, Seve P, Biard L, Baptiste Fraison J, Bielefeld P, Perard L, et al. Infliximab Versus Adalimumab in the Treatment of Refractory Inflammatory Uveitis: A Multicenter Study From the French Uveitis Network. Arthritis Rheumatol. 2016;68(6):1522-30.

68. Leccese P, Alpsoy E. Behçet's Disease: An Overview of Etiopathogenesis. Front Immunol. 2019;10:1067. 
69. Kuiper J, Rothova A, de Boer J, Radstake T. The immunopathogenesis of birdshot chorioretinopathy; a bird of many feathers. Prog Retin Eye Res. 2015;44:99-110.

70. Mercier A-E, Ribeiro E, Korobelnik J-F, Delyfer M-N, Rougier M-B. Efficacy of AntiTNF- $\alpha$ Therapy for the Treatment of Non-infectious Uveitis: A Retrospective Study of 21 Patients. Ocul Immunol Inflamm. 2016;0(0):1-8.

71. Ming S, Xie K, He H, Li Y, Lei B. Efficacy and safety of adalimumab in the treatment of non-infectious uveitis: a meta-analysis and systematic review. Drug Des Devel Ther. 2018;12:2005-16.

72. Martel JN, Esterberg E, Nagpal A, Acharya NR. Infliximab and Adalimumab for Uveitis. Ocul Immunol Inflamm. 2012;20(1):18-26.

73. Suhler EB, Adán A, Brézin AP, Fortin E, Goto H, Jaffe GJ, et al. Safety and Efficacy of Adalimumab in Patients with Noninfectious Uveitis in an Ongoing Open-Label Study: VISUAL III. Ophthalmology. 2018;125(7):1075-87.

74. Lejoyeux R, Diwo E, Vallet H, Saadoun D, Tezenas du Montcel S, Bodaghi B, et al. Infliximab and Adalimumab in Uveitic Macular Edema. Ocul Immunol Inflamm. 2018;26(7):991-6.

75. Maleki A, Sahawneh HF, Ma L, Meese H, He Y, Foster CS. Infliximab Therapy in Patients with Noninfectious Intermediate Uveitis resistant to Conventional Immunodulatory Therapy. Retina Phila Pa. 2017;37(5):836-43.

76. Jafri F, Sammut A. A rare case of suicidal ideation related to Adalimumab use. Open Access Rheumatol Res Rev. 2018;10:113-5.

77. Kappelmann N, Lewis G, Dantzer R, Jones PB, Khandaker GM. Antidepressant activity of anti-cytokine treatment: a systematic review and meta-analysis of clinical trials of chronic inflammatory conditions. Mol Psychiatry. 2018;23(2):335-43.

78. Fabiani C, Vitale A, Rigante D, Emmi G, Bitossi A, Lopalco G, et al. Comparative efficacy between adalimumab and infliximab in the treatment of non-infectious intermediate uveitis, posterior uveitis, and panuveitis: a retrospective observational study of 107 patients. Clin Rheumatol. 2019;38(2):407-415.

79. Deuter CME, Engelmann K, Heiligenhaus A, Lanzl I, Mackensen F, Ness T, et al. Enteric-coated mycophenolate sodium in the treatment of non-infectious intermediate uveitis: results of a prospective, controlled, randomised, open-label, early terminated multicentre trial. Br J Ophthalmol. 2018;102(5):647-53.

80. Pacheco PA, Taylor SRJ, Cuchacovich MT, Diaz GV. Azathioprine in the management of autoimmune uveitis. Ocul Immunol Inflamm. 2008;16(4):161-5.

81. Rathinam SR, Babu M, Thundikandy R, Kanakath A, Nardone N, Esterberg E, et al. A randomized clinical trial comparing methotrexate and mycophenolate mofetil for noninfectious uveitis. Ophthalmology. 2014;121(10):1863-70. 
82. Gangaputra SS, Newcomb CW, Joffe MM, Dreger K, Begum H, Artornsombudh P, et al. Comparison between Methotrexate and Mycophenolate Mofetil monotherapy for the control of Non- Infectious Ocular Inflammatory Diseases. Am J Ophthalmol. 2019;Epub ahead of print.

83. Samson CM, Waheed N, Baltatzis S, Foster CS. Methotrexate therapy for chronic noninfectious uveitis: analysis of a case series of 160 patients. Ophthalmology. 2001;108(6):1134-9.

84. Doycheva D, Zierhut M, Blumenstock G, Stuebiger N, Deuter C. Mycophenolate mofetil in the therapy of uveitic macular edema--long-term results. Ocul Immunol Inflamm. 2012;20(3):203-11.

85. Mackensen F, Jakob E, Springer C, Dobner BC, Wiehler U, Weimer P, et al. Interferon versus Methotrexate in Intermediate Uveitis With Macular Edema: Results of a Randomized Controlled Clinical Trial. Am J Ophthalmol. 2013;156(3):478-486.

86. Stone JH, Tuckwell K, Dimonaco S, Klearman M, Aringer M, Blockmans D, et al. Trial of Tocilizumab in Giant-Cell Arteritis. N Engl J Med. 2017;377(4):317-28.

87. Abásolo L, Rosales Z, Díaz-Valle D, Gómez-Gómez A, Peña-Blanco RC, Prieto-García Á, et al. Immunosuppressive Drug Discontinuation in Noninfectious Uveitis From Real-Life Clinical Practice: A Survival Analysis. Am J Ophthalmol. 2016;169:1-8.

88. Schaap-Fogler M, Amer R, Friling R, Priel E, Kramer M. Anti-TNF- $\alpha$ agents for refractory cystoid macular edema associated with noninfectious uveitis. Graefes Arch Clin Exp Ophthalmol Albrecht Von Graefes Arch Klin Exp Ophthalmol. 2014;252(4):633-40.

89. Lopalco G, Fabiani C, Sota J, Lucherini OM, Tosi GM, Frediani B, et al. IL-6 blockade in the management of non-infectious uveitis. Clin Rheumatol. 2017;36(7):1459-69.

90. Fardeau C, Champion E, Massamba N, LeHoang P. Uveitic macular edema. Eye Lond Engl. 2016;30(10):1277-92.

91. Suhler EB, Thorne JE, Mittal M, Betts KA, Tari S, Camez A, et al. CorticosteroidRelated Adverse Events Systematically Increase with Corticosteroid Dose in Noninfectious Intermediate, Posterior, or Panuveitis: Post Hoc Analyses from the VISUAL-1 and VISUAL-2 Trials. Ophthalmology. 2017;124(12):1799-807.

92. Kempen JH, Daniel E, Dunn JP, Foster CS, Gangaputra S, Hanish A, et al. Overall and cancer related mortality among patients with ocular inflammation treated with immunosuppressive drugs: retrospective cohort study. BMJ. 2009;339:2480.

93. Wolfe F, Michaud K. Biologic treatment of rheumatoid arthritis and the risk of malignancy: analyses from a large US observational study. Arthritis Rheum. 2007;56(9):2886-95.

94. Ozzello DJ, Palestine AG. Factors Affecting Therapeutic Decisions in Intermediate and Posterior Uveitis. Am J Ophthalmol. 2015;159(2):213-220. 
95. Urruticoechea-Arana A, Cobo-Ibáñez T, Villaverde-García V, Santos Gómez M, Loza E, Vargas-Osorio K, et al. Efficacy and safety of biological therapy compared to synthetic immunomodulatory drugs or placebo in the treatment of Behçet's disease associated uveitis: a systematic review. Rheumatol Int. 2019;39(1):47-58.

96. Atienza-Mateo B, Martín-Varillas JL, Calvo-Río V, Demetrio-Pablo R, Beltrán E, Sánchez-Bursón J, et al. Comparative study of infliximab versus adalimumab in refractory uveitis due to Behçet's disease, National multicenter study of 177 cases. Arthritis Rheumatol Hoboken NJ. 2019; Epub ahead of print.

97. Bhat P, Cervantes-Castañeda RA, Doctor PP, Anzaar F, Foster CS. Mycophenolate mofetil therapy for sarcoidosis-associated uveitis. Ocul Immunol Inflamm. 2009;17(3):185-90.

98. Bermejo I, Squires H, Poku EN, Cooper K, Stevens JW, Hamilton J, et al. Adalimumab for non-infectious uveitis: is it cost-effective? Br J Ophthalmol. 2019; Epub ahead of print.

99. Shakoor A, Esterberg E, Acharya NR. Recurrence of Uveitis after Discontinuation of Infliximab. Ocul Immunol Inflamm. 2014;22(2):96-101. 
"Pas un seul cheveu blanc n'a poussé sur mes rêves "

Hubert-Félix THIEFAINE Trois poèmes pour Annabel Lee Suppléments de mensonge 


\section{RESUME}

Introduction : Les uvéites non-infectieuses non-antérieures représentent une cause majeure de cécité dans les pays industrialisés. Aujourd'hui, en cas de rechute sous corticothérapie ou de cortico-dépendance, l'intensification thérapeutique repose sur les immunosuppresseurs conventionnels (méthotrexate, mycophénolate mofétil ou azathioprine). Cependant, avec l'émergence des biothérapies (anti-TNF alpha et anti-récepteur à l'IL-6), la stratégie thérapeutique actuelle est en train de se redéfinir.

Matériels et méthodes : Étude rétrospective monocentrique au CHU Rouen. Les patients majeurs présentant une uvéite intermédiaire, postérieure ou panuvéite non-infectieuse étaient divisés selon le traitement en cours lors de l'inclusion : groupe immunosuppresseur conventionnel ou groupe biothérapie. Le critère de jugement principal était un critère composite défini par l'apparition d'une rechute sous traitement ou l'arrêt du traitement pour un effet indésirable grave. La rechute était précisée selon des critères ophtalmologiques : majoration de l'inflammation intraoculaire et/ou détérioration de l'acuité visuelle et/ou absence d'amélioration de l'œdème maculaire ou d'une vascularite.

Résultats : Parmi les 1053 dossiers extraits, 79 patients ont été inclus, majoritairement de sexe féminin ( $51 \%$ ). On dénombrait $51 \%$ de panuvéites, $44 \%$ d'uvéites postérieures et $5 \%$ d'uvéites intermédiaires. Sur le plan diagnostique, il s'agissait principalement d'uvéites idiopathiques (31\%), de maladies de birdshot ( $25 \%)$, de sarcoïdoses (16\%) et de maladies de Behçet (13 $\%)$. Trente-six patients ont été inclus dans le groupe biothérapie et 39 dans le groupe immunosuppresseur conventionnel (ISc). Les patients du groupe biothérapie présentaient une maladie plus ancienne $(p=0,03)$ et avaient davantage rechuté $(p=0,01)$. Le critère de jugement principal a été atteint pour $56 \%$ des patients sous biothérapies versus $59 \%$ des patients du groupe ISc $(p=0,95)$, avec un délai médian de survenue de 16 mois versus 21 mois pour le groupe ISc $(p=0,47)$. Il n'y avait pas de différence entre les deux groupes en termes de survie sans progression, d'épargne cortisonique, d'amélioration de l'acuité visuelle, de correction de l'œdème maculaire ou d'effets indésirables. En revanche, les biothérapies étaient significativement plus efficaces pour assurer un contrôle précoce de l'inflammation oculaire (quiescence oculaire à 6 mois pour $92 \%$ des patients traités par biothérapies versus $68 \%$, $\mathrm{p}=0,046)$. A l'échelle individuelle, la cortico-dépendance au moment de la rechute était moins élevée sous biothérapies $(\mathrm{p}=0,001)$.

Conclusion : Notre étude montre que les immunosuppresseurs conventionnels doivent conserver leur place de première intention lors de l'intensification thérapeutique. Les biothérapies apparaissent comme une option intéressante en cas d'inflammation sévère, devant leur rapidité d'action. De plus, les biothérapies semblent plus efficaces pour assurer une épargne cortisonique à l'échelle individuelle et en cas de cortico-dépendance.

MOTS CLES : Uvéite non-infectieuse, Biothérapie, Immunosuppresseur conventionnel, Rechute, Tolérance. 